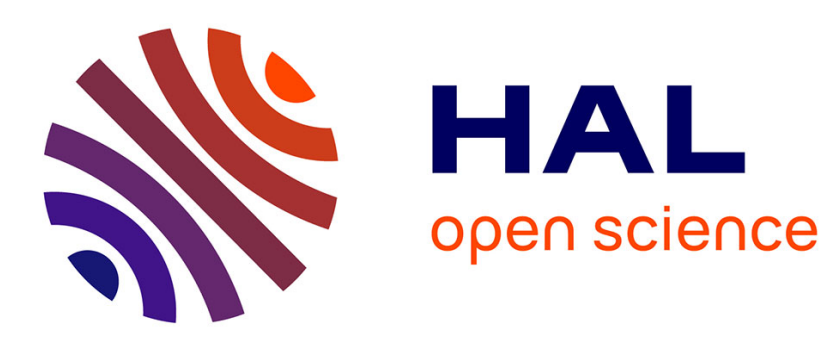

\title{
Well-posedness of general boundary-value problems for scalar conservation laws
}

Boris Andreianov, Karima Sbihi

\section{To cite this version:}

Boris Andreianov, Karima Sbihi. Well-posedness of general boundary-value problems for scalar conservation laws. Transactions AMS, 2014. hal-00708973v2

HAL Id: hal-00708973

https://hal.science/hal-00708973v2

Submitted on 31 Oct 2012 (v2), last revised 23 Jan 2015 (v3)

HAL is a multi-disciplinary open access archive for the deposit and dissemination of scientific research documents, whether they are published or not. The documents may come from teaching and research institutions in France or abroad, or from public or private research centers.
L'archive ouverte pluridisciplinaire HAL, est destinée au dépôt et à la diffusion de documents scientifiques de niveau recherche, publiés ou non, émanant des établissements d'enseignement et de recherche français ou étrangers, des laboratoires publics ou privés. 


\title{
WELL-POSEDNESS OF GENERAL BOUNDARY-VALUE PROBLEMS FOR SCALAR CONSERVATION LAWS
}

\author{
BORIS ANDREIANOV AND KARIMA SBIHI
}

\begin{abstract}
In this paper we investigate well-posedness for the problem $u_{t}+\operatorname{div} \varphi(u)=f$ on $(0, T) \times \Omega, \Omega \subset \mathbb{R}^{N}$, with initial condition $u(0, \cdot)=u_{0}$ on $\Omega$ and with general dissipative boundary conditions $\varphi(u) \cdot \nu \in \beta_{(t, x)}(u)$ on $(0, T) \times \partial \Omega$. Here for a.e. $(t, x) \in(0, T) \times \partial \Omega$, $\beta_{(t, x)}(\cdot)$ is a maximal monotone graph on $\mathbb{R}$. This includes, as particular cases, Dirichlet, Neumann, Robin, obstacle boundary conditions and their piecewise combinations.

As for the well-studied case of the Dirichlet condition, one has to interprete the formal boundary condition given by $\beta$ by replacing it with the adequate effective boundary condition. Such effective condition can be obtained through a study of the boundary layer appearing in approximation processes such as the vanishing viscosity approximation. We claim that the formal boundary condition given by $\beta$ should be interpreted as the effective boundary condition given by another monotone graph $\tilde{\beta}$, which is defined from $\beta$ by the projection procedure we describe. We give several equivalent definitions of entropy solutions associated with $\tilde{\beta}$ (and thus also with $\beta$ ).

For the notion of solution defined in this way, we prove existence, uniqueness and $L^{1}$ contraction, monotone and continuous dependence on the graph $\beta$. Convergence of approximation procedures and stability of the notion of entropy solution are illustrated by several results.
\end{abstract}

Keywords: scalar conservation law, boundary-value problem, entropy solution, vanishing viscosity limit, formal boundary condition, effective boundary condition, maximal monotone graph, strong boundary trace, $L^{1}$ contraction, well-posedness

\section{Contents}

1. Introduction

1.1. Dissipative boundary conditions for conservation laws 2

1.2. Classical results on the Dirichlet case 3

1.3. Strong traces of entropy solutions on the boundary 4

1.4. Interpretation of a general boundary condition $\quad 6$

$\begin{array}{ll}\text { 1.5. Former results and a summary of the paper } & 7\end{array}$

2. The effective BC graph 8

2.1. Preliminaries: undershoot and overshoot sets, increasing envelopes 8

2.2. Definition and equivalent characterizations of $\tilde{\beta} \quad 10$

2.3. Order and metric structure on $\mathbb{B}_{x} \quad 12$

3. Notion of solution $\quad 13$

3.1. Equivalent definitions of entropy solutions, sub-solutions, super-solutions 13

3.2. Proof of the equivalence of different definitions $\quad 15$

4. Uniqueness, comparison, continuous dependence 18

5. Existence: a formal proof 19

6. Justification of the effective boundary condition 21

2010 Mathematics Subject Classification. Primary 35L65, 35L04; Secondary 35A01, 35A02.

The work of the first author was partially supported by the French ANR projet CoToCoLa. 
6.1. Convergence of the vanishing viscosity approximation 21

6.2. Stability of the notion of entropy solution $\quad 25$

7. Further existence and convergence results 30

7.1. The one-dimensional case: existence via $B V_{l o c}$ estimate 30

7.2. Entropy-process solutions $\quad 31$

Conclusion $\quad 34$

Appendix: existence for the viscosity regularized problem $\quad 34$

References $\quad 37$

\section{INTRODUCTION}

While there exists an extensive literature on the Cauchy and Cauchy-Dirichlet problems for scalar conservation law $u_{t}+\operatorname{div} \varphi(u)=0$, other initial-boundary value problems have received very few attention. This is the purpose of this paper to define a notion of entropy solution for a wide class of boundary conditions that we call dissipative boundary conditions; to justify this definition through convergence of natural approximation procedures; and to establish wellposedness results for the so defined entropy solutions.

1.1. Dissipative boundary conditions for conservation laws. Let $\Omega$ be an open domain in $\mathbb{R}^{N}$ with Lipschitz boundary, $N \geq 1$, and $T>0$. We consider the following initial-boundary value problem for a scalar conservation law:

$$
\left(H_{\varphi, \beta}\left(u_{0}, f\right)\right) \quad \begin{cases}u_{t}+\operatorname{div} \varphi(u)=f & \text { in } Q_{T}:=(0, T) \times \Omega \\ \left.u\right|_{t=0}=u_{0} & \text { in } \Omega \\ \varphi_{\nu(x)}(u):=\varphi(u) \cdot \nu(x) \in \beta_{(t, x)}(u) & \text { on } \Sigma:=(0, T) \times \partial \Omega .\end{cases}
$$

Here $\varphi: \mathbb{R} \longrightarrow \mathbb{R}^{N}$ is locally a continuous function (for the sake of simplicity, the reader may assume that $\varphi$ is Lipschitz continuous, although most of our results hold without this assumption); $u_{0} \in L^{\infty}(\Omega)$; and $f$ is a measurable function on $Q_{T}$ with $\int_{0}^{T}\|f(t, \cdot)\|_{L^{\infty}(\Omega)}<\infty$.

Further, in $\left(H_{\varphi, \beta}\left(u_{0}, f\right)\right)$, the unit outward normal vector on $\partial \Omega$ is denoted by $\nu$, and the boundary condition is prescribed (formally) in terms of $\beta$ that is a map from $\Sigma$ to the set $\mathbb{B}$ of all maximal monotone graphs on $\mathbb{R}$. Clearly, some measurability assumption is needed on the $\operatorname{map} \beta:(t, x) \in \Sigma \mapsto \beta_{(t, x)} \in \mathbb{B}$. In the sequel, we always extend $\beta_{(t, x)}$ to a maximal monotone graph from $\overline{\mathbb{R}}$ to $\overline{\mathbb{R}}$ and require the following:

$$
\begin{aligned}
& \text { for all } k \in \mathbb{R},(t, x) \mapsto \inf \beta_{(t, x)}(k) \text { and }(t, x) \mapsto \sup \beta_{(t, x)}(k) \\
& \text { are measurable } \overline{\mathbb{R}} \text {-valued functions wrt the Hausdorff measure on } \Sigma \text {. }
\end{aligned}
$$

This encompasses different classical boundary conditions. For instance, the graph $\beta_{(t, x)}=$ $\left\{u^{D}(t, x)\right\} \times \mathbb{R}$ prescribes the Dirichlet boundary condition " $u=u^{D}$ on $\Sigma$ "; the graph $\beta_{(t, x)}:=$ $\mathbb{R} \times\{-g(t, x)\}$ prescribes the condition " $-\varphi(u) \cdot \nu(x)=g$ " that we will call Neumann condition, by analogy with the Neumann boundary conditions for the general convection-diffusion problems of the kind $u_{t}-\operatorname{div} a(u, \nabla u)=f$. It is also easy to include the more general conditions of the kind $\lambda u+(1-\lambda)(-\varphi(u) \cdot \nu)=g, \lambda \in(0,1)$, conditions that interpolate between the Dirichlet and the Neumann ones (these are known as Robin conditions in the convection-diffusion context). To give one more example, the (bilateral) obstacle boundary conditions " $u^{m} \leq u \leq u^{M}$ on $\Sigma$ " correspond to the graph

$$
\beta_{(t, x)}=\left(\left\{u^{m}(t, x)\right\} \times \mathbb{R}^{-}\right) \cup\left(\left[u^{m}(t, x), u^{M}(t, x)\right] \times\{0\}\right) \cup\left(\left\{u^{M}(t, x)\right\} \times \mathbb{R}^{+}\right) .
$$


For the sake of simplicity, the reader may consider

$$
\begin{aligned}
& \beta_{(t, x)}(r)=\beta_{(t, x)}^{0}\left(r-u^{D}(t, x)\right)-g(t, x) \text { with } u^{D} \in L^{\infty}(\Sigma), g \in L^{\infty}(\Sigma) \\
& \text { and with a maximal monotone graph } \beta_{(t, x)}^{0} \text { such that } \beta_{(t, x)}^{0}(0) \ni 0 ;
\end{aligned}
$$

this contains the aforementioned cases and, e.g., the case of mixed Dirichlet-Neumann boundary conditions.

In the context of parabolic problems $u_{t}-\operatorname{div} a(u, \nabla u)=f$, it is well known that the boundary conditions of the kind $\beta_{(t, x)}(u)+a(u, \nabla u) \cdot \nu(x) \ni 0$ lead to the $L^{1}$ contraction property (see e.g. [37] for a study of the associated stationary elliptic problem; see also [3]); that's why we call these conditions dissipative boundary conditions. It is customary to interprete the physically admissible weak solutions (called entropy solutions since the founding work [20] of Kruzhkov) of a scalar conservation law as limits of the vanishing viscosity approximation that, in our case, would take the form

$$
\left\{\begin{array}{l}
u_{t}^{\varepsilon}-\operatorname{div}\left(-\varphi\left(u^{\varepsilon}\right)+\varepsilon \nabla u^{\varepsilon}\right)=f,\left.\quad u^{\varepsilon}\right|_{t=0}=u_{0}, \\
\left.\left(\beta_{(t, x)}\left(u^{\varepsilon}\right)+\left(-\varphi\left(u^{\varepsilon}\right)+\varepsilon \nabla u^{\varepsilon}\right) \cdot \nu(x)\right)\right|_{(t, x) \in \Sigma} \ni 0 .
\end{array}\right.
$$

Then it is clear that the boundary condition in $\left(H_{\varphi, \beta}\left(u_{0}, f\right)\right)$ is the formal limit of the dissipative boundary condition $\beta_{(t, x)}\left(u^{\varepsilon}\right)+\left(-\varphi\left(u^{\varepsilon}\right)+\varepsilon \nabla u^{\varepsilon}\right) \cdot \nu(x) \ni 0$ in (1.3) (here we should assume some regularity of $\beta_{(t, x)}$ in $(t, x)$ in order that a solution $u^{\varepsilon}$ exist; for instance, for the Dirichlet $\mathrm{BC}$ case we need $\left.u^{D} \in L^{2}\left(0, T ; H^{-1 / 2}(\partial \Omega)\right)\right)$. Moreover let $u^{\varepsilon}, \hat{u}^{\varepsilon}$ be solutions of problem (1.3) with the same dissipative boundary condition and with data $u_{0}, f$ and $\hat{u}_{0}, \hat{f}$, respectively. The $L^{1}$ contraction property holds under rather weak restrictions on $\Omega$ and $\varphi$ (see, e.g., [25, 5]):

$$
\left\|u^{\varepsilon}(t, \cdot)-\hat{u}^{\varepsilon}(t, \cdot)\right\|_{L^{1}(\Omega)} \leq\left\|u_{0}-\hat{u}_{0}\right\|_{L^{1}(\Omega)}+\|f-\hat{f}\|_{L^{1}(\Omega)}
$$

Provided the $L^{1}\left(Q_{T}\right)$ compactness of the sequences $\left(u^{\varepsilon}\right)_{\varepsilon},\left(\hat{u}^{\varepsilon}\right)_{\varepsilon}$ with $\varepsilon \rightarrow 0$ is known, it is inherited at the limit $\varepsilon \rightarrow 0$. Therefore we expect that the boundary condition satisfied at the limit is also a dissipative one.

But what is this limit boundary condition as $\varepsilon \rightarrow 0$ in (1.3) ? The compactness of $\left(u^{\varepsilon}\right)_{\varepsilon}$ in $L^{1}\left(Q_{T}\right)$ gives no information on convergence of $u^{\varepsilon}$ on the boundary, the term $\varepsilon \nabla u^{\varepsilon} \cdot \nu(x)$ on the boundary becomes singular as $\varepsilon \rightarrow 0$, therefore passage to the limit in boundary conditions is by no means straightforward. As a matter of fact, in general

the boundary condition " $\varphi(u) \cdot \nu(x) \in \beta_{(t, x)}(u)$ " is not the correct limit obtained from the boundary conditions $\beta_{(t, x)}\left(u^{\varepsilon}\right)+\left(-\varphi\left(u^{\varepsilon}\right)+\varepsilon \nabla u^{\varepsilon}\right) \cdot \nu(x) \ni 0$.

The Dirichlet condition case discussed below is a well-known illustration of this fact.

1.2. Classical results on the Dirichlet case. Within the whole variety of dissipative boundary conditions, only the Dirichlet case received much attention in the framework of conservation laws. The celebrated result of Bardos, LeRoux and Nédélec [9] states that the Dirichlet condition " $u=u^{D}$ on $\Sigma$ " should be seen as a formal condition; and that it must be interpreted by stating that the trace $(\gamma u)(t, x)$ of $u$ at a point $(t, x) \in \Sigma$ belongs to the subset $I(t, x)$ of $\mathbb{R}$ defined in terms of $u^{D}(t, x)$ and of the function $r \mapsto \varphi_{\nu(x)}(r)=\varphi(r) \cdot \nu(x)$ as follows:

$$
\begin{aligned}
& I(t, x)=\left\{z \in \mathbb{R} \mid \operatorname{sign}\left(z-u^{D}(t, x)\right)\left(\varphi_{\nu(x)}(z)-\varphi_{\nu(x)}(k)\right) \geq 0\right. \\
&\left.\forall k \in\left[u^{D}(t, x) \wedge z, u^{D}(t, x) \vee z\right]\right\} .
\end{aligned}
$$

Here and in the sequel, $\wedge$ (respectively, $\vee$ ) denotes the min (resp., the max) operation. We denote by $\mathcal{H}^{N}$ the $N$-dimensional Hausdorff measure on $\Sigma$. 
The effective boundary condition

$$
(\gamma u)(t, x) \in I(t, x) \mathcal{H}^{N} \text {-a.e. on } \Sigma
$$

is known as the BLN condition; in this paper, we will use the reformulation of the BLN condition in terms of a maximal monotone (sub)graph. Such graph interpretation was first made explicit, for the Dirichlet case, by Dubois and LeFloch in [17] (see in particular [17, Fig.1.1]).

The functional framework of the paper [9] is the space $L^{\infty}(0, T ; B V(\Omega))$ (actually, the solutions belong to the space $\left.B V\left(Q_{T}\right)\right)$. There are two good reasons for that. Firstly, the $B V$ in space regularity of $u$ guarantees the existence of a trace $\gamma u$ of $u$ on $\Sigma$, necessary in order to give sense to the BLN condition. Secondly, uniform in $\varepsilon B V$ estimates on the solutions of the approximating problems (1.3) are available, for $B V$ data $u_{0}$ and $u^{D}$ and for Lipschitz continuous flux function $\varphi$. Bardos, LeRoux and Nédélec show that for the above data and flux, there exists a unique $L^{\infty}(0, T ; B V(\Omega))$ entropy solution of the conservation law satisfying (pointwise on $\Sigma$ ) the BLN boundary condition; and that this solution is the limit of the vanishing viscosity approximation.

More recently, Otto in [26, 27] (see also [24]) provided a formulation suitable for merely $L^{\infty}$ data $u_{0}$ and $u^{D}$; Porretta, Vovelle [34] and Ammar, Carrillo and Wittbold [2] extended the definition and results to the framework of $L^{1}$ data (see the papers for the precise assumptions on $u^{D}$ ) and merely continuous flux function $\varphi$, in a bounded domain $\Omega$. The $L^{1}$ framework requires an appropriate notion of solution; in [34, 2] the notion of renormalized solution from [10] was used. In the Otto formulation, existence of a (strong) boundary trace $\gamma u$ of $u$ on $\Sigma$ is not assumed; a BLN kind condition is reformulated in terms of weak normal boundary traces of $\varphi(u)$ and of the associated boundary entropy fluxes $\mathcal{F}\left(u ; u^{D}, k\right)$ (the existence of the weak traces is a relatively simple consequence of the fact that $u$ is a Kruzhkov entropy solution of the scalar conservation law inside $(0, T) \times \Omega)$. We refer to [26, 27, 24] and to [40,34, 38] for details and results related to the approach of Otto.

1.3. Strong traces of entropy solutions on the boundary. Although the definition of $[26,27]$ and the aforementioned generalizations were a remarkable step forward in the study of boundary value problems for conservation laws, it was possible to bypass the use of weak traces and the associated boundary entropies' techniques of [26, 27]. Indeed, for the sake of simplicity let us start with the following flux non-degeneracy assumption:

$$
\forall \xi \in \mathbb{R}^{N} \backslash\{0\} \forall c \in \mathbb{R} \text { the Lebesque measure of the set }\{z \mid \xi \cdot \varphi(z)=c\} \text { is zero. }
$$

Using the approach of kinetic solutions (see [23,33]), Vasseur in [39] has shown that for $\varphi$ regular enough,

(1.7) under (1.6), any $L^{\infty}$ Kruzhkov entropy solution in $Q_{T}$ admits a strong trace $\gamma u$ on $\Sigma$.

The non-degeneracy assumption (1.6) on $\varphi$ is typical for the "compactification properties" in the theory of kinetic solutions, see Perthame [33] and references therein. As pointed out by Vasseur, (1.7) gives sense to the pointwise BLN condition (1.5) for general $L^{\infty}$ entropy solutions, and not only to solutions corresponding to $B V$ data; thus the weak trace technique of Otto [26, 27] is bypassed (yet for general $(t, x)$-dependent flux $\varphi$, the approach of $[26,27]$ remains the most powerful; see in particular the results of Vallet [38]). Further results in the spirit of (1.7) were obtained by Kwon and Vasseur [22] for the case $N=1$ (see also [6, 36] where we treat the case of a flat boundary using a hint due to Panov). To the authors' knowledge, the strongest generalization of (1.7) is the result of Panov [31] obtained using the technique of parametrized families of $H$-measures (see also [29, 32]); Panov drops all regularity assumption on $\varphi$, and, in a sense, he also drops non-degeneracy assumptions of the kind (1.6). Because of its importance for our paper, we should make the latter statement more precise: 
- (upon rotating axes and localizing around a point $x^{*}$ of the boundary) the boundary $\partial \Omega$ is represented by the graph of a Lipschitz ${ }^{1}$ function $g$ on $W$, i.e.,

$\partial \Omega \cap U=\left\{\left(g\left(x^{\prime}\right), x^{\prime}\right) \mid x^{\prime} \in W\right\}, \quad \Omega \cap U=\left\{\left(x_{0}, x^{\prime}\right) \mid x_{0}=y+g\left(x^{\prime}\right), x^{\prime} \in W, y \in(0, h)\right\}$

for some neighbourhood $U$ of $x^{*}$, some neighbourhood $V$ of zero in $R^{N-1}$, and some $h>0$; further, the unit exterior normal field $\left(\nu\left(g\left(x^{\prime}\right), x^{\prime}\right)\right)_{x^{\prime} \in W}$ is lifted inside $\Omega \cap U$ by the formula $\nu\left(x_{0}, x^{\prime}\right)=\frac{1}{\sqrt{1+\left|\nabla g\left(x^{\prime}\right)\right|^{2}}}\left(-1, \nabla g\left(x^{\prime}\right)\right)$ (the field is constant in $x_{0} \in[0, h)$;

- for $x \in \partial \Omega \cap U$, consider the singular mapping $V \varphi_{\nu(x)}: r \mapsto \int_{0}^{r}\left|\varphi^{\prime}(s) \cdot \nu(x)\right| d s$ on $\mathbb{R}$ (notice that the mapping is independent of $x_{0}$, and it depends on $x^{\prime}$ continuously)

- then for any $u \in L^{\infty}\left(Q_{T}\right)$ that is a Kruzhkov entropy solution in $Q_{T}$, there exists

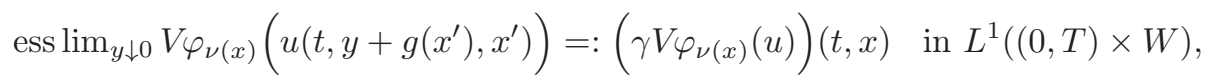

where $x:=\left(g\left(x^{\prime}\right), x^{\prime}\right)$ is a generic point of $U \cap \partial \Omega$; recall that $\nu\left(y+g\left(x^{\prime}\right), x^{\prime}\right) \equiv \nu\left(g\left(x^{\prime}\right), x^{\prime}\right)$.

Statement (1.8) is actually a re-interpretation of the localization property that appears in the proof [31, p.571] of Panov; we use it to give a sense to pointwise formulations of boundary conditions, in the same vein as Vasseur in [39]. If for all $\xi \in \mathbb{R}^{N} \backslash\{0\}$ the function $r \mapsto \varphi(r) \cdot \xi$ is non-constant on any interval (this is a weaker version of (1.6) typical for the technique of parametrized $H$-measures, see $[29,31,32]$ ), then $V \varphi_{\nu\left(x_{0}\right)}$ is an invertible function (which means that strong trace $\gamma u$ exists). If $\varphi$ is not a $B V$ function, one can use another singular mapping instead of the map $r \mapsto \int_{0}^{r}\left|\varphi^{\prime}(z) \cdot \nu\left(x_{0}\right)\right| d z$ (which is not well defined), e.g.,

$$
V \varphi_{\nu(x)}(r)=\int_{0}^{r} 1_{F}(s) d s, \quad \begin{aligned}
& F \text { being the union of all the intervals } \\
& \text { where the map } s \mapsto \varphi(s) \cdot \nu(x) \text { does not vary. }
\end{aligned}
$$

Remark 1.1. By the definition of the singular mapping, $V \varphi_{\nu(x)}(\cdot)$ has the properties of being monotone non-decreasing and of being constant on the same intervals where $\varphi_{\nu(x)}(\cdot)$ is constant. Therefore $\varphi(r) \cdot \nu(x)=\Phi_{\nu(x)} \circ V \varphi_{\nu(x)}$ with some continuous function $\Phi_{\nu(x)}: \mathbb{R} \rightarrow \mathbb{R}$. As a consequence of (1.8), there exists the strong trace $\gamma \varphi(u) \cdot \nu(x)$ (with the same meaning as in (1.8)) which is equal to $\Phi_{\nu(x)}\left(\gamma V \varphi_{\nu(x)}(u)\right)$.

In the same way, one can represent the projections on the direction $\nu(x)$ of the semi-Kruzhkov entropy fluxes

$$
q^{ \pm}(u, k):=\operatorname{sign}^{ \pm}(u-k)(\varphi(u)-\varphi(k))
$$

with the help of continuous functions $Q_{\nu(x)}^{ \pm}(\cdot, \cdot)$ of two variables:

$$
q^{ \pm}(u, k) \cdot \nu(x)=Q_{\nu(x)}^{ \pm}\left(V \varphi_{\nu(x)}(u), V \varphi_{\nu(x)}(k)\right)
$$

Hence for a couple $u, \hat{u}$ of entropy solutions, it follows that a strong trace of $q^{ \pm}(u, \hat{u}) \cdot \nu(x)$ exists and can be represented as $Q_{\nu(x)}^{ \pm}\left(\gamma V \varphi_{\nu(x)}(u), \gamma V \varphi_{\nu(x)}(\hat{u})\right)$. The same is true for the Kruzhkov fluxes:

$$
\begin{aligned}
& q(u, k) \cdot \nu(x)=Q_{\nu(x)}\left(V \varphi_{\nu(x)}(u), V \varphi_{\nu(x)}(k)\right) \\
& \text { with } q:=q^{+}+q^{-}, Q_{\nu(x)}:=Q_{\nu(x)}^{+}+Q_{\nu(x)}^{-} .
\end{aligned}
$$

\footnotetext{
${ }^{1}$ While the setting of Panov [31] is $C^{1}$ regular domains, the author indicates that the generalization to Lipschitz and, more generally, Lipschitz deformable boundaries in the sense of [16] is straightforward
} 
1.4. Interpretation of a general boundary condition. The Bardos-LeRoux-Nédélec condition (1.4),(1.5) is generally recognized as the correct interpretation of the Dirichlet boundary condition; this is justified in particular by convergence of vanishing viscosity or numerical approximations of the boundary value problem (see Vovelle [40]), considered as quite natural approximations. Observations of viscous or numerical boundary layers explain how the formal boundary condition $u=u^{D}$ on $\Sigma$ transforms into the effective boundary condition (1.4),(1.5).

The strong trace result of [39] was used by Bürger, Frid and Karlsen in [12] in order to give sense to the formal zero-flux boundary condition (in our terminology, this is the Neumann boundary condition with $g \equiv 0$ ) in the particular but important case $\varphi(0)=0=\varphi(1)$. Under this assumption and for $[0,1]$-valued initial data, the zero-flux boundary condition for $u_{t}+\operatorname{div} \varphi(u)=0$ can be understood literally (see [12]) (in the sense that the problem is well-posed and solutions are limits of the vanishing viscosity approximation).

Let us stress that in general, also for the zero-flux boundary condition " $\varphi(u) \cdot \nu=0$ " a boundary layer would form in approximate solutions, and this formal zero-flux boundary condition would transform into some different effective boundary condition. For a simple example, consider the zero-flux problem for the transport equation $u_{t}+u_{x}=0$ on [0,1]; as in [9], arguing along characteristics one sees that the zero-flux condition (that reads " $u=0$ " because $\varphi=I d$ ) at the right boundary $x=1$ must be merely dropped.

It is the purpose of this paper to provide a natural interpretation for a general dissipative boundary condition (formally given by a family $\beta$ of maximal monotone graphs $\beta_{(t, x)}(\cdot)$ ) under the form of an effective boundary condition. Most generally, this effective boundary condition can be written under the form

$$
\mathcal{H}^{N} \text {-a.e. on } \Sigma \text {, the couple }\left(\gamma V \varphi_{\nu} u, \varphi\left(\gamma V \varphi_{\nu} u\right) \cdot \nu\right) \text { lies in the graph } \tilde{\beta}_{(t, x)}(\cdot) \circ\left(V \varphi_{\nu}\right)^{-1}
$$

with $\tilde{\beta}$ to be defined, and with the notation $\gamma V \varphi_{\nu} u:=\left(\gamma V \varphi_{\nu(x)}(u)\right)(t, x)$.

To clarify the essence of the condition (1.12), consider the case where $V \varphi_{\nu(x)}=I d$ can be taken (recall that this is the case if (1.6) holds). Then (1.12) means that $(\gamma u)(t, x) \in \operatorname{Dom} \tilde{\beta}_{(t, x)}(\cdot)$; and from the definition of $\tilde{\beta}$ in Section 2 we will see that this automatically includes the equality $\tilde{\beta}_{(t, x)}(\gamma u(t, x))=\varphi(\gamma u(t, x)) \cdot \nu$. Thus the condition " $\varphi(u) \cdot \nu(x)=\tilde{\beta}_{(t, x)}(u)$ on $\Sigma$ " can be understood literally as a pointwise equality; this is why we call it effective boundary condition. Notice that condition (1.12) takes the form " $\left(\gamma V \varphi_{\nu} u\right)(t, x) \in V \varphi_{\nu}(I(t, x))$ a.e. on $\Sigma$ ", i.e., it prescribes some set $I(t, x)$ of possible trace values of $u$ on the boundary. Recall that the BLN condition (1.4) has the same form.

The effective BC graph $\tilde{\beta}_{(t, x)}$ featuring in (1.12) will be characterized in Section 2 as:

$\tilde{\beta}_{(t, x)}$ is the "closest" to $\beta_{(t, x)}$ maximal monotone subgraph of the graph $\{(r, \varphi(r) \cdot \nu(x)) \mid r \in \mathbb{R}\}$ that contains all the points of crossing of $\beta_{(t, x)}$ with the graph of the function $\varphi_{\nu(x)}=\varphi \cdot \nu(x)$.

For simplicity, let us look at the case where $\varphi$ is a $C^{1}$ function; then the monotonicity of $\tilde{\beta}_{(t, x)}$ means that the domain of the graph contains either isolated points $r \in \mathbb{R}$ such that $\varphi(r) \cdot \nu(x) \in$ $\beta_{(t, x)}(r)$, or intervals where $\varphi^{\prime}(\cdot) \cdot \nu(x) \geq 0$. Therefore heuristically, (1.12) can be understood as 
follows (assume for simplicity that $\gamma u$ exists):

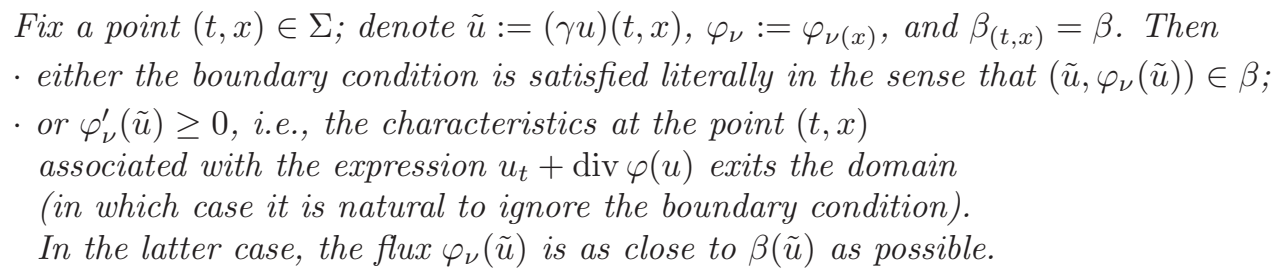

In view of the description $\left(B_{\tilde{\beta}}\right)$ of $\tilde{\beta}$, the interpretation of the formal $\mathrm{BC}$ " $\varphi_{\nu}(\tilde{u}) \in \beta(\tilde{u})$ " as " $\varphi_{\nu}(\tilde{u})=\tilde{\beta}(\tilde{u})$ " can appear as a rather natural one. Yet the only convincing justification we can think about would be in terms of approximation. Namely, we should use the formal boundary condition given by $\beta$ on one of the approximation schemes that are well-established in the context of conservation laws (such as the vanishing viscosity approximation or approximation with a monotone consistent finite volume scheme); pass to the limit in the sequence of the approximate solutions; and identify the boundary condition satisfied at the limit. If this can be achieved only for some restricted class of "regular" data $u_{0}, f$, graphs $\beta$ or fluxes $\varphi$, then a further justification can be provided by a passage to the limit from the "regular" problem (where the correct BC is already identified) to the general problem.

1.5. Former results and a summary of the paper. Beyond the Cauchy-Dirichlet problem described in Section 1.2 and the "simple case" of the zero-flux problem treated in [12], we are not aware of works on initial-boundary value problems for conservation laws.

The present paper develops the approach initiated in the thesis [36] of K. Sbihi; see [6, 7]. The graph $\tilde{\beta}$ (in a different, but equivalent representation, see Section 2) was introduced in $[6,36]$. The passage from $\beta$ to $\tilde{\beta}$ was justified in $[6,36]$ in the case of a flat boundary, of nondegenerate in the sense (1.6) flux $\varphi$ and for quickly growing at infinity, $(t, x)$-independent graph $\beta$. A combination of vanishing viscosity method and nonlinear semigroup methods were used in this argument. Notice that the technique of $[6,36]$ is rather restrictive because it is based upon a strong compactness on the boundary of the sequence of approximate solutions. In [7], the definition of $\tilde{\beta}$ was further supported through an argument of monotone dependence on $\beta$; a notion of measure-valued (or entropy-process) solution was introduced, in order to simplify the convergence analysis for different approximation methods.

Let us give an outline of the paper. Proposition 3.3 and Theorems 4.1,5.2 are its main results.

In Section 2, we discuss in detail the properties and different characterizations of the projected graph $\tilde{\beta}$; this long section can be omitted by a reader convinced by the heuristic arguments of Section 1.4 and not interested in details of some proofs. In Section 3 we provide several equivalent definitions of entropy solutions, sub- and super-solutions for the formal problem $\left(H_{\varphi, \beta}\left(u_{0}, f\right)\right)$. These definitions lead, in a rather straightforward way, to uniqueness, comparison and continuous dependence results proved in Section 4 under minimal restrictions on $\beta$ and $\varphi$.

In the existence part of the paper, several restrictions on the behaviour of $\varphi$ and $\beta$ are needed for ensuring boundedness and compactness of sequences of approximate solutions. In Section 5, we give a short but somewhat artificial proof of existence of entropy solutions (namely, we use not $\beta$ but the projected graphs $\tilde{\beta}$ to construct approximate solutions). In Section 6 , we discuss in length the pertinence of the use of $\tilde{\beta}$. First, we justify the appearance of the effective boundary condition using the vanishing viscosity parabolic approximation recalled in the Appendix. Second, we give several stability results for entropy solutions on the hyperbolic problem $\left(H_{\varphi, \beta}\left(u_{0}, f\right)\right)$, with a focus on stability with respect to different approximations of the $\mathrm{BC}$ graphs $\beta$. In Section 7, first we improve the existence results in the one-dimensional case, 
dropping most of the assumptions on $\varphi$ and $\beta$ with the help of the $B V_{l o c}$ estimates due to Bürger, Karlsen, García and Towers [13, 14]. Second, following Eymard, Gallouët and Herbin [19] we present a notion of entropy-process solution that is useful in order to prove convergence of approximations with only weak compactness properties; it can be exploited under the additional, quite restrictive assumption that an entropy solution exists already.

\section{The EFFeCtive BC GRAPH}

Throughout the section, we fix a point $(t, x) \in \Sigma$. We are given a maximal monotone graph $\beta_{(t, x)}$ on $\mathbb{R}$ and a continuous function $\varphi_{\nu(x)}$ on $\mathbb{R}$; the associated "semi-Kruzhkov" entropy fluxes (more precisely, their normal components) are defined as

$$
q_{\nu(x)}^{ \pm}(z, k):=\operatorname{sign}^{ \pm}(z-k)\left(\varphi_{\nu(x)}(z)-\varphi_{\nu(x)}(k)\right) .
$$

2.1. Preliminaries: undershoot and overshoot sets, increasing envelopes. Let us start with a series of definitions and notation.

Definition 2.1 (see Figure 2.1 for an illustration).

- For a closed sub-interval $I$ of $\mathbb{R}$, introduce the upper increasing envelope $e^{2} \varphi_{x}^{+}(I ; \cdot)$ and the lower increasing envelope $\varphi_{x}^{-}(I ; \cdot)$ of $\varphi_{\nu(x)}$ on $I$ by setting, for $r \in I$,

$$
\begin{aligned}
& \varphi_{x}^{+}(I ; r):=\inf \left\{\psi(r) \mid \psi \geq \varphi_{\nu(x)} \text { and } \psi \text { is non-decreasing on } I\right\}, \\
& \varphi_{x}^{-}(I ; r):=\sup \left\{\psi(r) \mid \psi \leq \varphi_{\nu(x)} \text { and } \psi \text { is non-decreasing on } I\right\} .
\end{aligned}
$$

- Define the overshoot set $D_{(t, x)}^{+} \subset \mathbb{R}$ and the undershoot set $D_{(t, x)}^{-} \subset \mathbb{R}$ by ${ }^{3}$

$$
\left.\begin{array}{l}
D_{(t, x)}^{+}:=\left\{\begin{array}{l}
z \in \mathbb{R} \mid \sup \beta_{(t, x)}(z) \geq \varphi_{\nu(x)}(z) \\
D_{(t, x)}^{-}
\end{array}:=\{,\right. \\
z \in \mathbb{R} \mid \inf \beta_{(t, x)}(z) \leq \varphi_{\nu(x)}(z)
\end{array}\right\} ;
$$

also introduce the crossing set $^{4} D_{(t, x)}^{0}:=\left\{r \in \mathbb{R} \mid \varphi_{\nu(x)}(r) \in \beta_{(t, x)}(r)\right\} \equiv D_{(t, x)}^{+} \cap D_{(t, x)}^{-}$.

- Subgraphs of the graph of $\varphi_{\nu(x)}$ are defined as the graphs of restrictions of $\left.\varphi_{\nu(x)}\right|_{E}$ on different subsets $E$ of $\mathbb{R}$. Among these, we distinguish monotone subgraphs characterized by the property $\varphi_{\nu(x)}(a) \leq \varphi_{\nu(x)}(b)$ for all $a, b \in E$ with $a \leq b$. Finally, those monotone subgraphs that does not possess a nontrivial extension (within the class of monotone subgraphs) are called maximal monotone subgraphs of the graph of $\varphi_{\nu(x)}$.

- Denote by $\mathbb{B}_{x}$ the set of all maximal monotone subgraphs of the graph of $\varphi_{\nu(x)}$. Denote by $\mathbb{B}_{(t, x)}^{0}$ the set of all elements of $\mathbb{B}_{x}$ which domain contains $D_{(t, x)}^{0}$.

- Denote by $\overline{\mathbb{B}}_{x}$ (respectively, by $\overline{\mathbb{B}_{(t, x)}^{0}}$ ) the set of all maximal monotone graphs on $\mathbb{R}$ obtained as extensions of elements of $\mathbb{B}_{x}$ (respectively, of $\left.\mathbb{B}_{(t, x)}^{0}\right)^{6}$.

\footnotetext{
${ }^{2}$ It is easily seen that $\varphi_{x}^{+}(I ; \cdot)$, respectively $\varphi_{x}^{-}(I ; \cdot)$ is a non-decreasing function that is continuous and which graph lies above (respectively, below) from the graph of $\left.\varphi_{\nu(x)}\right|_{I}$.

${ }^{3}$ In definition (2.4), we actually extend $\beta_{(t, x)}$ to a maximal monotone graph from $\overline{\mathbb{R}}$ to $\overline{\mathbb{R}}$, so that $\beta_{(t, x)}(z)$ is never empty but it may de reduced to $\{+\infty\}$ or to $\{-\infty\}$. With this convention, $\mathbb{R}=D_{(t, x)}^{+} \cup D_{(t, x)}^{-}$.

${ }^{4}$ Indeed, $D_{(t, x)}^{0}$ is the set of crossing points of $\beta_{(t, x)}$ with the graph of $\varphi_{\nu(x)}$.

${ }^{5}$ It follows that for any $\mu \in \mathbb{B}_{(t, x)}^{0},\left\{\left(z, \varphi_{\nu(x)}(z)\right) \mid z \in D_{(t, x)}^{0}\right\} \subset \mu \subset\left\{\left(z, \varphi_{\nu(x)}(z)\right) \mid z \in \mathbb{R}\right\}$.

${ }^{6}$ First, it easily follows from the continuity of $\varphi_{\nu(x)}$ and the intermediate value theorem that for each $\mu \in \mathbb{B}_{x}$ there exists a unique extension $\bar{\mu} \in \overline{\mathbb{B}}_{x}$. The graph $\bar{\mu}$ is actually the graph of a single-valued continuous function on $\mathbb{R}$; moreover, on every connected component $(a, b)$ of the set $\left\{z \in \mathbb{R} \mid \bar{\mu}(z) \neq \varphi_{\nu(x)}(z)\right\}$ the function $\bar{\mu}$ takes the constant value equal to the value of $\varphi_{\nu(x)}$ on $\{a, b\} \cap \mathbb{R}$.
} 
- Define the monotone function $\widetilde{\mathcal{B}}_{(t, x)}$ on $\mathbb{R}$ as the closest to $\beta_{(t, x)}$ element of $\overline{\mathbb{B}_{(t, x)}^{0}}$.

The notion of "the closest" in the latter definition should be made precise: indeed, we now show that the definition of $\widetilde{\mathcal{B}}_{(t, x)}$ is correct, interpreted as the extremality property $(2.5)$.

Proposition 2.2. The function $\widetilde{\mathcal{B}}_{(t, x)}$ is correctly defined, in the sense that

there exists $\widetilde{\mathcal{B}}_{(t, x)} \in \overline{\mathbb{B}}_{(t, x)}^{0}$ that realizes, simultaneously for all $z \in \mathbb{R}$, the minimum over all $\bar{\mu} \in \overline{\mathbb{B}_{(t, x)}^{0}}$ of the distance dist $\left(\bar{\mu}(z), \beta_{(t, x)}(z)\right)$.

Furthermore, $\widetilde{\mathcal{B}}_{(t, x)}$ can be expressed in terms of the upper (respectively, lower) increasing envelopes of the graph of $\varphi_{\nu(x)}$ on the connected components ${ }^{7} I$ of $D_{(t, x)}^{+}$(respectively, of $\left.D_{(t, x)}^{-}\right)$:

$$
\begin{aligned}
\widetilde{\mathcal{B}}_{(t, x)}:= & \left(\bigcup_{I}\left\{\left(z, \varphi_{x}^{-}(I ; z)\right) \mid I \text { is a connected component of } D_{(t, x)}^{-}\right\}\right) \\
& \bigcup\left(\bigcup_{I}\left\{\left(z, \varphi_{x}^{+}(I ; z)\right) \mid I \text { is a connected component of } D_{(t, x)}^{+}\right\}\right) .
\end{aligned}
$$

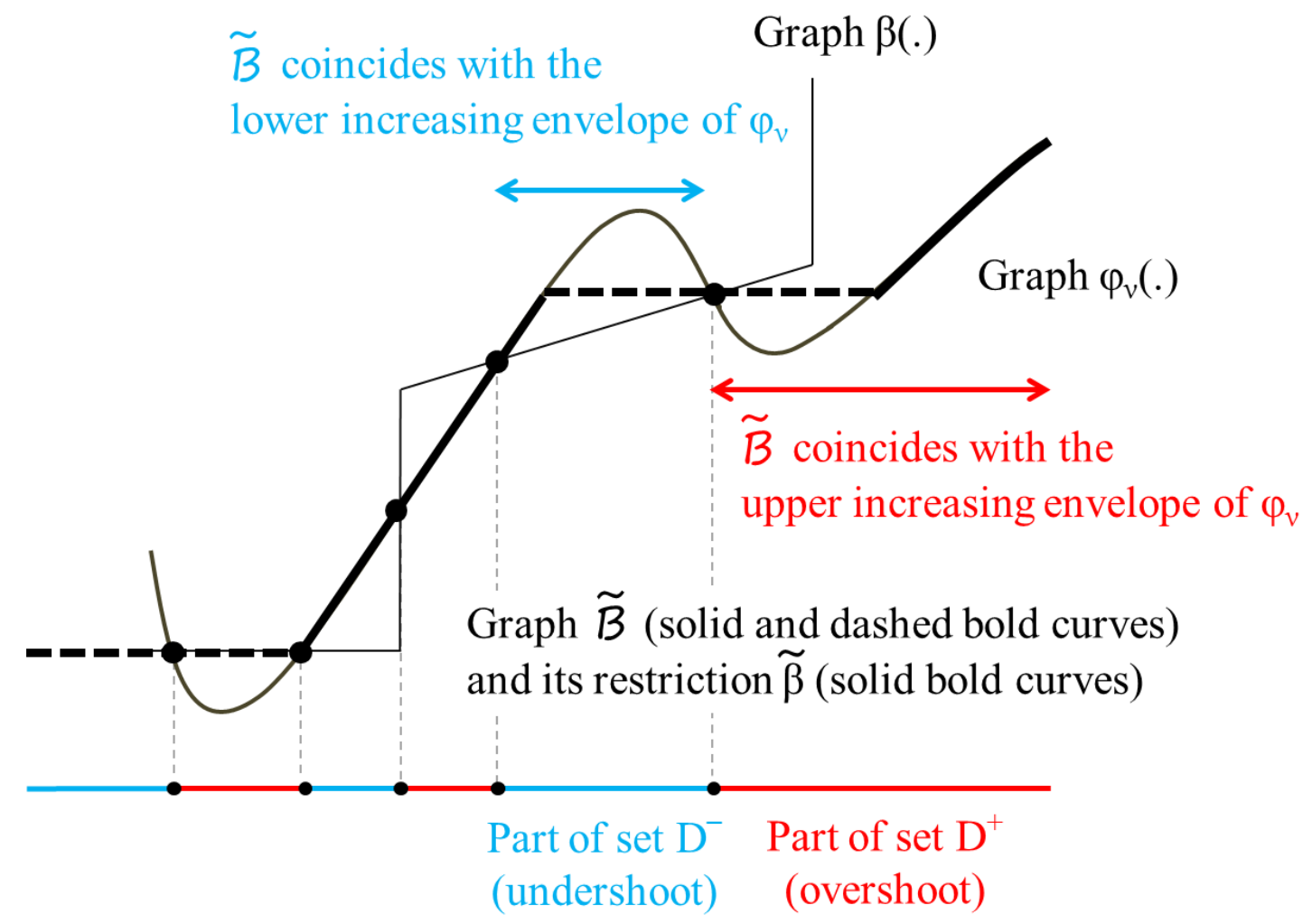

FiguRE 1. Example of construction of the projected graph $\widetilde{\beta}$

\footnotetext{
${ }^{7}$ Let us recall that $\mathrm{I}$ is a connected component of $K \subset \mathbb{R}$ if $I$ is an interval and moreover, for all interval $J$ such that $I \subset J \subset K$, one has $J=I$.
} 
Proof. By definition on the class $\overline{\mathbb{B}_{(t, x)}^{0}},\left.\widetilde{\mathcal{B}}_{(t, x)}\right|_{D_{(t, x)}^{0}}$ coincides with $\left.\varphi_{\nu(x)}\right|_{D_{(t, x)}^{0}}$. Let $I$ be a connected component of $D_{(t, x)}^{+}$or of $D_{(t, x)}^{-}$; the endpoints of $I$ are either infinite or belong to $D_{(t, x)}^{0}$. Therefore, we only need to make explicit the definition of $\widetilde{\mathcal{B}}_{(t, x)}$ on the interior of $I$; and for a proof of (2.5) we can consider $z \in I$ separately for every connected component $I$ of $D_{(t, x)}^{+}$ or of $D_{(t, x)}^{-}$. To be specific, consider $I \subset D_{(t, x)}^{+}$. From (2.2), one easily sees that

$$
\varphi_{\nu(x)} \leq \varphi_{x}^{+}(I ; \cdot) \leq \beta \text { on } I .
$$

Every function $\bar{\mu} \in \overline{\mathbb{B}_{(t, x)}^{0}}$ is constant on each interval where it does not coincide with $\varphi_{\nu(x)}$, while $\varphi_{x}^{+}(I ; \cdot)$ and $\beta$ are monotone on these intervals. Thus from $(2.7)$ it follows that

$$
\forall \bar{\mu} \in \overline{\mathbb{B}}_{(t, x)}^{0} \quad \bar{\mu} \leq \varphi_{x}^{+}(I ; \cdot) \leq \beta \text { on } I .
$$

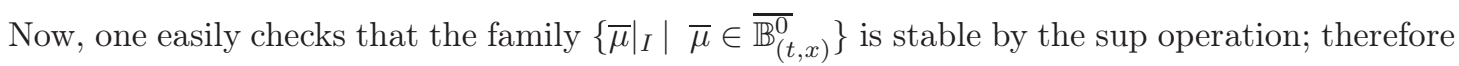
it possesses a greatest element that we call $\psi$. This element is the restriction of $\widetilde{\mathcal{B}}_{(t, x)}$ on $I$.

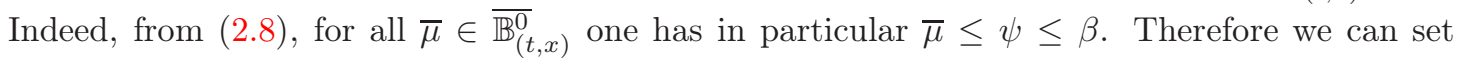
$\left.\widetilde{\mathcal{B}}_{(t, x)}\right|_{I}:=\psi$, and $(2.5)$ gets verified for all $z \in I$.

We have seen in $(2.8)$ that $\left.\widetilde{\mathcal{B}}_{(t, x)}\right|_{I}=\psi \leq \varphi_{x}^{+}(I ; \cdot)$. The function $\psi$ is non-decreasing and its graph lies above $\left.\varphi_{\nu(x)}\right|_{I}$ by $(2.7)$; thus according to $(2.2), \psi \geq \varphi_{x}^{+}(I ; \cdot)$. Therefore $\left.\widetilde{\mathcal{B}}_{(t, x)}\right|_{I}$ coincides with $\varphi_{x}^{+}(I ; \cdot)$, for every connected component $I$ of $D_{(t, x)}^{+}$or of $D_{(t, x)}^{-}$. This yields (2.6).

\subsection{Definition and equivalent characterizations of $\tilde{\beta}$.}

Definition 2.3. The graph $\tilde{\beta}_{(t, x)}$ is the part of $\widetilde{\mathcal{B}}_{(t, x)}$ contained within the graph of $\varphi_{\nu(x)}$.

It is clear from the above definition that $\tilde{\beta}_{(t, x)} \in \mathbb{B}_{(t, x)}^{0}$. Namely, $\tilde{\beta}_{(t, x)}$ is a maximal monotone subgraph of $\varphi_{\nu(x)}$ containing the crossing points with $\beta_{(t, x)}$. Moreover, the unique extension $\widetilde{\mathcal{B}}_{(t, x)}$ of $\tilde{\beta}_{(t, x)}$ to a maximal monotone graph on $\mathbb{R}$ satisfies $(2.5)$. Thus Definition 2.3 is a precise expression of $\left(A_{\tilde{\beta}}\right)$, in view of the extremality property $(2.5)$.

Notice that, according to $(2.6), \beta_{(t, x)}$ intervenes in the construction of $\tilde{\beta}_{(t, x)}$ uniquely through the sets $D_{(t, x)}^{ \pm}$that gather the points $z \in \mathbb{R}$ such that $\pm\left(\beta_{(t, x)}(z)-\varphi_{\nu(x)}(z)\right) \cap \mathbb{R}^{+} \neq \emptyset$.

Remark 2.4. The operation $\widetilde{\mathcal{P}}_{x}$ that transforms the maximal monotone graph maximal $\beta_{(t, x)}$ into the maximal monotone graph $\widetilde{\mathcal{B}}_{(t, x)}$ is a projection on $\mathbb{B}_{x}$. Indeed, we have $\widetilde{\mathcal{P}}_{x}^{2}=\widetilde{\mathcal{P}}_{x}$ and $\left.\widetilde{\mathcal{P}}_{x}\right|_{\mathbb{B}_{x}}=I d$. With a slight abuse of notation (the graph $\tilde{\beta}_{(t, x)}=\left.\widetilde{\mathcal{B}}_{(t, x)}\right|_{\operatorname{Dom} \tilde{\beta}_{(t, x)}}$ being monotone but not necessarily maximal $)^{8}$, we will say that the operation $\sim: \beta_{(t, x)} \mapsto \tilde{\beta}_{(t, x)}$ is a projection.

Let us give alternative characterizations of $\tilde{\beta}_{(t, x)}$. Recall that $\tilde{\beta}_{(t, x)}$ is a subgraph of the graph of $\varphi_{\nu(x)}$, thus it is fully characterized by its domain.

Proposition 2.5. The domain of the graph $\tilde{\beta}_{(t, x)}$ given by Definition 2.3 can be equivalently defined by any of the following properties:

\footnotetext{
${ }^{8}$ Actually, uniqueness of solutions to the boundary value problems with dissipative boundary condition encoded by a graph $\beta$ stems from the monotonicity of $\beta$ only. Existence may depend on how wide is the domain of $\beta$. In the sequel we will see that the monotone graph $\tilde{\beta}$, while it is not maximal, leads to existence and uniqueness for the problem in hand.
} 
(i) In terms of the semi-Kruzhkov entropy fluxes (2.1), one has

$$
\operatorname{Dom} \tilde{\beta}_{(t, x)}=\left\{z \in \mathbb{R} \mid\left(\forall k \in D_{(t, x)}^{-} q_{\nu}^{-}(z, k) \geq 0\right) \&\left(\forall k \in D_{(t, x)}^{+} q_{\nu}^{+}(z, k) \geq 0\right)\right\} \text {. }
$$

(ii) For $z \in \mathbb{R}$, denote $\beta_{(t, x)}^{-1}\left(\varphi_{\nu(x)}(z)\right)=:\left[m_{(t, x)}(z), M_{(t, x)}(z)\right]$; this is a non-empty ${ }^{9}$ closed interval of $\overline{\mathbb{R}}$. Notice that $z<m_{(t, x)}(z)$ (resp., $z>M_{(t, x)}(z)$ ) for $z \in D_{(t, x)}^{-} \backslash D_{(t, x)}^{0}$ (resp., for $\left.z \in D_{(t, x)}^{-} \backslash D_{(t, x)}^{0}\right)$. With this notation, we have

$$
\begin{aligned}
\operatorname{Dom} \tilde{\beta}_{(t, x)} & =D_{(t, x)}^{0} \\
& \bigcup\left\{z \in D_{(t, x)}^{-} \backslash D_{(t, x)}^{0} \mid \varphi_{\nu(x)}(k) \geq \varphi_{\nu(x)}(z) \forall k \in\left[z, m_{(t, x)}(z)\right]\right\} \\
& \bigcup\left\{z \in D_{(t, x)}^{+} \backslash D_{(t, x)}^{0} \mid \varphi_{\nu(x)}(k) \leq \varphi_{\nu(x)}(z) \forall k \in\left[M_{(t, x)}(z), z\right]\right\} .
\end{aligned}
$$

Remark 2.6. Characterization (i), in its spirit, goes back to the idea of Carrillo [15] further developed by Ammar, Carrillo and Wittbold [2], for the case of the Dirichlet problem. In [15], $u^{D}=0$ and thus $\beta(t, x ; \cdot)=\{0\} \times \mathbb{R} ;$ therefore $D_{(t, x)}^{ \pm}=\mathbb{R}^{ \pm}$in this case. In [2], $\beta_{(t, x)}=$ $\left\{u^{D}(t, x)\right\} \times \mathbb{R}$ and thus $D_{(t, x)}^{-}=\left(-\infty, u^{D}(t, x)\right], D_{(t, x)}^{+}=\left[u^{D}(t, x),+\infty\right)$. Further, notice that for the Dirichlet boundary condition, $\inf \beta_{(t, x)}^{-1}\left(\varphi_{\nu(x)}(z)\right)=u^{D}(t, x)=\sup \beta_{(t, x)}^{-1}\left(\varphi_{\nu(x)}(z)\right)$. Thus we see that characterization (ii) is precisely the Bardos-LeRoux-Nédélec set (1.4). Representation (ii) of $\tilde{\beta}_{(t, x)}$ is therefore a generalization of the BLN condition; it appeared in the previous works $[36]$ and $[6,7]$ of the authors (see in particular [6, formula (4)]).

Proof. Throughout the proof, we write $\widetilde{D}_{(t, x)}^{0}:=\operatorname{Dom} \tilde{\beta}_{(t, x)}=\left\{z \in \mathbb{R} \mid \widetilde{\mathcal{B}}_{(t, x)}(z)=\varphi_{\nu(x)}(z)\right\}$.

(i) Let us assume that $z \in \widetilde{D}_{(t, x)}^{0}$. Consider, e.g., $k \in D_{(t, x)}^{-}$: according to (2.5) in this case we have $\varphi_{\nu(x)}(k) \geq \widetilde{\mathcal{B}}_{(t, x)}(k)$. Then

$$
q_{\nu}^{-}(z, k)=\operatorname{sign}^{-}(z-k)\left(\varphi_{\nu(x)}(z)-\varphi_{\nu(x)}(k)\right) \geq \operatorname{sign}^{-}(z-k)\left(\widetilde{\mathcal{B}}_{(t, x)}(z)-\widetilde{\mathcal{B}}_{(t, x)}(k)\right) \geq 0
$$

by the monotonicity of $\widetilde{\mathcal{B}}_{(t, x)}$. The case $k \in D_{(t, x)}^{-}$is analogous.

Reciprocally, assume that for all $k \in D_{(t, x)}^{ \pm}$one has $q_{\nu}^{ \pm}(z, k) \geq 0$. For the sake of being definite, assume that $z$ belongs to a connected component $I$ of $D_{(t, x)}^{+}$. Let $k \in I, k<z$; by assumption we have $\varphi_{\nu(x)}(k) \leq \varphi_{\nu(x)}(z)$ for all such $k$. Keeping in mind the characterization (2.6) in Proposition 2.2, we see that $\varphi_{x}^{+}(k)=\varphi_{\nu(x)}(k)$ if and only if $k \in \widetilde{D}_{(t, x)}^{0}$. It follows that $\tilde{u}$ verifies $\varphi_{x}^{+}(k) \leq \varphi_{\nu(x)}(z)$ for all $k \in I, k<z$. By the definition of the upper increasing envelope $\varphi_{x}^{+}$, this exactly means that $\varphi_{\nu(x)}(z)=\varphi_{x}^{+}(z)$. Hence $z \in \widetilde{D}_{(t, x)}^{0}$.

(ii) The case $z \in D_{(t, x)}^{0}$ is trivial. Let $I$ be the connected component of the complementary of $D_{(t, x)}^{0}$ that contains $z$; for the sake of being definite, assume that $I \subset D_{(t, x)}^{+}$. Then from the monotonicity of $\beta_{(t, x)}$ we have

$$
p h_{\nu(x)}(k) \leq \sup \beta_{(t, x)}(k) \leq \beta_{(t, x)}\left(M_{(t, x)}(z)\right) \ni \varphi_{\nu(x)}(z)
$$

for $k \in I \cap\left(-\infty, M_{(t, x)}(z)\right)$. By the characterization (2.6) of $\widetilde{\mathcal{B}}_{(t, x)}$ on $I, z \in \widetilde{D}_{(t, x)}^{0} \cap I$ if and only if $\varphi_{\nu(x)}(k) \leq \varphi_{\nu(x)}(z)$ for all $k \in I, k \leq z$. Taking into account (2.9), we can reformulate this as follows: $z \in \widetilde{D}_{(t, x)}^{0} \cap I$ if and only if, firstly, $\left[M_{(t, x)}(z), z\right] \subset I$ and secondly, $\varphi_{\nu(x)}(k) \leq \varphi_{\nu(x)}(z)$ for all $k \in\left[M_{(t, x)}(z), z\right]$. This justifies the statement of (ii).

\footnotetext{
$9_{\text {we mean that }} \tilde{\beta}_{(t, x)}$ is extended to a maximal monotone graph on $\overline{\mathbb{R}}$
} 
2.3. Order and metric structure on $\mathbb{B}_{x}$. Fix $x \in \partial \Omega$. Recall that $\mathbb{B}_{x}$ is the set of all maximal monotone subgraphs of $\varphi_{\nu(x)} ; \mathbb{B}$ is the set of all maximal monotone graphs of $\overline{\mathbb{R}}$ and $\overline{\mathbb{B}}_{x}$ is the subset of $\mathbb{B}$ obtained by extension (which is unique) from $\operatorname{Dom} \tilde{\beta}$ to $\mathbb{R}$ of elements $\tilde{\beta} \in \mathbb{B}_{x}$.

Let us define an order relation and a distance for maximal monotone graphs under study. They are most naturally defined on $\overline{\mathbb{B}}_{x}$.

Definition 2.7. For $\widetilde{\mathcal{B}}^{1}, \widetilde{\mathcal{B}}^{2} \in \overline{\mathbb{B}}_{x}$, define the uniform distance

$$
d_{x}\left(\widetilde{\mathcal{B}}^{1}, \widetilde{\mathcal{B}}^{2}\right):=\left\|\widetilde{\mathcal{B}}^{1}-\widetilde{\mathcal{B}}^{2}\right\|_{\infty}=\sup _{\mathbb{R}}\left|\widetilde{\mathcal{B}}^{1}-\widetilde{\mathcal{B}}^{2}\right| .
$$

Define the order relation " $\succeq_{x}$ " on $\overline{\mathbb{B}}_{x}$ by:

$$
\widetilde{\mathcal{B}}^{1} \succeq_{x} \widetilde{\mathcal{B}}^{2} \quad \text { if } \quad \widetilde{\mathcal{B}}^{1} \geq \widetilde{\mathcal{B}}^{2} \text { pointwise on } \mathbb{R}
$$

Since every $\tilde{\beta} \in \mathbb{B}_{x}$ possesses a unique extension $\widetilde{\mathcal{B}}^{2} \in \overline{\mathbb{B}}_{x}$, we can define $d_{x}$ and $\succeq_{x}$ on $\mathbb{B}_{x}$ by writing, e.g., $d_{x}\left(\tilde{\beta}^{1}, \tilde{\beta}^{2}\right):=d_{x}\left(\widetilde{\mathcal{B}}^{1}, \widetilde{\mathcal{B}}^{2}\right)$. Further, every $\beta \in \mathbb{B}$ gives rise to the projection $\widetilde{\mathcal{B}}:=\widetilde{\mathcal{P}}_{x} \beta$ on $\mathbb{B}_{x}$. Thus we can extend $d_{x}$ to a semi-distance on $\beta \in \mathbb{B}$; and we can extend $\succeq_{x}$ to a binary relation on $\mathbb{B}$ by assigning $\beta^{1} \succeq_{x} \beta^{2}$ whenever $\widetilde{\mathcal{P}}_{x} \beta \succeq_{x} \widetilde{\mathcal{P}}_{x} \beta$.

In Sections 4, 6.2.2, 6.2.3 we will use these definitions in combination with the following lemma.

Lemma 2.8. One can represent the distance $d_{x}(\cdot, \cdot)$ by the formulas

$$
\begin{aligned}
d_{x}\left(\tilde{\beta}^{1}, \tilde{\beta}^{2}\right) & =\sup _{a, b \in \mathbb{R}} \operatorname{sign}(b-a)\left(\widetilde{\mathcal{B}}^{1}(a)-\widetilde{\mathcal{B}}^{2}(b)\right) \\
& =\sup \left\{\operatorname{sign}(b-a)\left(\varphi_{\nu}(a)-\varphi_{\nu}(b)\right) \mid a \in \operatorname{Dom}\left(\tilde{\beta}^{1}\right), b \in \operatorname{Dom}\left(\tilde{\beta}^{2}\right)\right\} .
\end{aligned}
$$

One can express the relation $\beta^{1} \succeq_{x} \beta^{2}$ through the formula $d_{x}^{-}\left(\tilde{\beta}^{1}, \tilde{\beta}^{2}\right)=0$, where

$$
\begin{aligned}
d_{x}^{-}\left(\tilde{\beta}^{1}, \tilde{\beta}^{2}\right) & :=\sup _{a, b \in \mathbb{R}} \operatorname{sign}^{-}(b-a)\left(\widetilde{\mathcal{B}}^{1}(a)-\widetilde{\mathcal{B}}^{2}(b)\right)=\sup \left\{\left(\widetilde{\mathcal{B}}^{1}(a)-\widetilde{\mathcal{B}}^{2}(b)\right)^{-} \mid a>b\right\} \\
& =\sup \left\{\left(\varphi_{\nu}(a)-\varphi_{\nu}(b)\right)^{-} \mid a \in \operatorname{Dom}\left(\tilde{\beta}^{1}\right), b \in \operatorname{Dom}\left(\tilde{\beta}^{2}\right), a>b\right\} \\
& =\sup \left\{\left(Q_{\nu(x)}^{+}(\tilde{a}, \tilde{b})\right)^{-} \mid \tilde{a} \in \operatorname{V\varphi } \varphi_{\nu(x)} \operatorname{Dom}\left(\tilde{\beta}^{1}\right), \tilde{b} \in \operatorname{Vi} \varphi_{\nu(x)} \operatorname{Dom}\left(\tilde{\beta}^{2}\right)\right\},
\end{aligned}
$$

where we have used the singular mapping $V \varphi_{\nu(x)}$ and the notation of Section 1.3.

Proof. On the one hand, from the monotonicity of $\widetilde{\mathcal{B}}^{2}$, we have

$$
\begin{array}{r}
\sup _{a, b \in \mathbb{R}} \operatorname{sign}(b-a)\left(\widetilde{\mathcal{B}}^{1}(a)-\widetilde{\mathcal{B}}^{2}(b)\right)=\sup _{a, b \in \mathbb{R}} \operatorname{sign}(b-a)\left(\widetilde{\mathcal{B}}^{1}(a)-\widetilde{\mathcal{B}}^{2}(a)+\widetilde{\mathcal{B}}^{2}(a)-\widetilde{\mathcal{B}}^{2}(b)\right) \\
\leq\left|\widetilde{\mathcal{B}}^{1}(a)-\widetilde{\mathcal{B}}^{2}(a)\right| \leq\left\|\widetilde{\mathcal{B}}^{1}-\widetilde{\mathcal{B}}^{2}\right\|_{\infty}=d_{x}\left(\tilde{\beta}^{1}, \tilde{\beta}^{2}\right) .
\end{array}
$$

On the other hand, consider $a=k$ and $b_{n}=k+\frac{1}{n}$, then $b_{n}=k-\frac{1}{n}$ in the left-hand side of the above expression, with $n \rightarrow \infty$. Using the continuity of $\widetilde{\mathcal{B}}^{2}$ we get for all $k \in \mathbb{R}$,

$$
\left|\widetilde{\mathcal{B}}^{1}(k)-\widetilde{\mathcal{B}}^{2}(k)\right| \leq \sup _{a, b \in \mathbb{R}} \operatorname{sign}(b-a)\left(\widetilde{\mathcal{B}}^{1}(a)-\widetilde{\mathcal{B}}^{2}(b)\right) .
$$

Hence we derive the first equality in (2.10). Further, recall that $\widetilde{\mathcal{B}}^{i}$ is constant on each connected component of the complementary of $\operatorname{Dom} \tilde{\beta}^{i}$, while $\left.\widetilde{\mathcal{B}}^{i}\right|_{\operatorname{Dom} \tilde{\beta}^{i}}=\left.\varphi_{\nu}\right|_{\operatorname{Dom} \tilde{\beta}^{i}}$; this implies the second equality in (2.10).

In the same way, we justify the first three equalities in (2.11). The last equality in (2.11) is evident from the definitions of $V \varphi_{\nu(x)}$ and $Q_{\nu(x)}^{+}$. 


\section{Notion OF SOLUTION}

Let us start with the following notation. Given $\beta_{(t, x)} \in \mathbb{B}$, in the previous section we have constructed its projection $\widetilde{\mathcal{B}}_{(t, x)} \in \overline{\mathbb{B}}_{x}$. Then we write

$$
\begin{gathered}
\widetilde{D}_{(t, x)}^{-}:=\left\{k \in \mathbb{R} \mid \widetilde{\mathcal{B}}_{(t, x)}(k) \leq \varphi_{\nu(x)}(k)\right\} \equiv D_{(t, x)}^{-} \cup \operatorname{Dom} \tilde{\beta}_{(t, x)} ; \\
\widetilde{D}_{(t, x)}^{+}:=\left\{k \in \mathbb{R} \mid \widetilde{\mathcal{B}}_{(t, x)}(k) \geq \varphi_{\nu(x)}(k)\right\} \equiv D_{(t, x)}^{+} \cup \operatorname{Dom} \tilde{\beta}_{(t, x)} ; \\
\widetilde{D}_{(t, x)}^{0}:=\operatorname{Dom} \tilde{\beta}_{(t, x)} \equiv \widetilde{D}_{(t, x)}^{-} \cap \widetilde{D}_{(t, x)}^{+} .
\end{gathered}
$$

Recall that $D_{(t, x)}^{-}, D_{(t, x)}^{+}$and $D_{(t, x)}^{0}$ are the undershoot, the overshoot and the crossing sets for the graph $\beta_{(t, x)}$ given the normal flux $\varphi_{\nu(x)}$; similarly, $\widetilde{D}_{(t, x)}^{-}, \widetilde{D}_{(t, x)}^{+}$and $\widetilde{D}_{(t, x)}^{0}$ are the undershoot, the overshoot and the crossing sets for the projected graph $\widetilde{\mathcal{B}}_{(t, x)}$. These sets appear as sets of boundary traces of entropy sub-solutions, super-solutions and solutions, respectively, according to the definitions we now give.

Note the following localized version of the celebrated definition of entropy solution due to Kruzhkov [20]. Recall that $q^{ \pm}(\cdot, \cdot)$ are the semi-Kruzhkov entropy fluxes defined by (1.9).

Definition 3.1. Let $\widehat{Q_{T}}$ be an open subdomain of $Q_{T}=(0, T) \times \Omega$. A function $u \in L^{\infty}\left(\widehat{Q_{T}}\right)$ is called entropy solution of problem $u_{t}+\operatorname{div} \varphi(u)=f,\left.u\right|_{t=0}=u_{0}$ if for all $k \in \mathbb{R}$, for all $\xi \in \mathcal{D}\left(\widehat{Q_{T}} \cup(\{0\} \times \Omega)\right)^{+}$

$$
\int_{0}^{T} \int_{\Omega}\left(-(u-k)^{ \pm} \xi_{t}-q^{ \pm}(u, k) \cdot \nabla \xi\right)-\int_{\Omega}\left(u_{0}-k\right)^{ \pm} \xi(0, \cdot) \leq \int_{0}^{T} \int_{\Omega} \operatorname{sign}^{ \pm}(u-k) f \xi .
$$

If only the sign "plus" (respectively, "minus") is chosen in (3.1), then $u$ is an entropy sub-solution (respectively, an entropy super-solution) in $\widehat{Q_{T}}$.

Remark 3.2. Notice that entropy solutions, sub- and super-solutions are quasi-solutions in the sense of Panov (see [31]). This implies that the boundary traces in the sense of Section 1.3, used in the definitions of the next section, do exist. It should also be noted that, according to the result of [30] (see also [39]), entropy solutions in the whole cylinder $Q_{T}$ actually belong to $C\left(0, T ; L_{l o c}^{1}(\Omega)\right)$, in particular the initial datum $u_{0}$ is assumed in the sense of strong $L_{l o c}^{1}$ trace.

3.1. Equivalent definitions of entropy solutions, sub-solutions, super-solutions. Now we include into the definition the boundary condition. We need one more notation:

$$
\Sigma^{ \pm}(k):=\left\{(t, x) \in \Sigma \mid k \in D_{(t, x)}^{ \pm}\right\} .
$$

In order to describe simultaneously the key features of entropy solutions, we gather a series of equivalent definitions in the following Definition and Proposition.

Proposition 3.3 (Definition of an entropy solution). Let $u \in L^{\infty}\left(Q_{T}\right)$.

If any of the below items (i)-(iv) is satisfied, $u$ is called an entropy solution of problem $\left(H_{\varphi, \beta}\left(u_{0}, f\right)\right)$. Indeed, the assertions (i)-(iv) are equivalent:

(i) The function $u$ verifies the entropy inequalities (3.1) with $\xi \in \mathcal{D}([0, T) \times \Omega)^{+}$, moreover, for $\mathcal{H}^{N}$-a.e. $(t, x) \in \Sigma$, the strong trace $\gamma V \varphi_{\nu(x)} u$ belongs to the set $V \varphi_{\nu(x)} \widetilde{D}_{(t, x)}^{0}$.

(ii) The function $u$ verifies the entropy inequalities (3.1) with $\xi \in \mathcal{D}([0, T) \times \Omega)^{+}$, moreover, for $\mathcal{H}^{N}$-a.e. $(t, x) \in \Sigma$, the strong trace $\gamma V \varphi_{\nu(x)} u$ verifies

$$
\forall k \in \widetilde{D}_{(t, x)}^{0} \quad Q_{\nu(x)}\left(\gamma V \varphi_{\nu(x)} u, V \varphi_{\nu(x)} k\right) \geq 0
$$


Here, according to (1.11), $Q_{\nu(x)}$ represents the normal component of the Kruzhkov entropy flux $q(u, k)=\operatorname{sign}(u-k)(\varphi(u)-\varphi(k))$.

(iii) The function $u$ verifies the entropy inequalities (3.1) with $\xi \in \mathcal{D}([0, T) \times \Omega)^{+}$, moreover, for $\mathcal{H}^{N}$-a.e. $(t, x) \in \Sigma$, the strong trace $\gamma V \varphi_{\nu(x)} u$ verifies

$$
\forall k \in D_{(t, x)}^{ \pm} \quad Q_{\nu(x)}^{ \pm}\left(\gamma V \varphi_{\nu(x)} u, V \varphi_{\nu(x)} k\right) \geq 0
$$

Here, $Q_{\nu(x)}^{ \pm}$are defined by (1.10).

(iv) The function $u$ verifies the up-to-the-boundary entropy inequalities with remainder term:

$$
\begin{aligned}
& \forall k \in \mathbb{R} \quad \forall \xi \in \mathcal{D}([0, T) \times \bar{\Omega})^{+} \\
& \int_{0}^{T} \int_{\Omega}\left(-(u-k)^{ \pm} \xi_{t}-q^{ \pm}(u, k) \cdot \nabla \xi\right)-\int_{\Omega}\left(u_{0}-k\right)^{ \pm} \xi(0, \cdot) \\
& \quad \leq \int_{0}^{T} \int_{\Omega} \operatorname{sign}^{ \pm}(u-k) f \xi+\iint_{\Sigma} C_{k} \wedge\left(\beta_{(t, x)}(k)-\varphi_{\nu(x)}(k)\right)^{\mp} \xi(t, x) .
\end{aligned}
$$

Here, $C_{k}$ is a constant ${ }^{10}$ that depends on $\|u\|_{\infty}$ and on $k$.

Moreover, if the sets $\Sigma^{ \pm}(k)$ in (3.2) are regular enough in the sense that ${ }^{11}$

$$
\begin{aligned}
& \text { for a countable dense set of values of } k, \\
& \text { the space } \mathcal{D}\left(\Sigma^{ \pm}(k)\right) \text { in dense in } L^{1}\left(\Sigma^{ \pm}(k)\right) \text {, }
\end{aligned}
$$

then (i)-(iv) are also equivalent to

(v) The function $u$ verifies the following up-to-the-boundary entropy inequalities:

$$
\begin{gathered}
\forall k \in \mathbb{R} \quad \forall \xi \in \mathcal{D}([0, T) \times \bar{\Omega})^{+} \text {such that }\left.\xi\right|_{\Sigma \backslash \Sigma^{ \pm}(k)}=0 \\
\int_{0}^{T} \int_{\Omega}\left(-(u-k)^{ \pm} \xi_{t}-q^{ \pm}(u, k) \cdot \nabla \xi\right)-\int_{\Omega}\left(u_{0}-k\right)^{ \pm} \xi(0, \cdot) \leq \int_{0}^{T} \int_{\Omega} \operatorname{sign}^{ \pm}(u-k) f \xi .
\end{gathered}
$$

Remark 3.4. Let us provide a few comments to the different items of Proposition 3.3 and their use for establishing well-posedness for problem $\left(H_{\varphi, \beta}\left(u_{0}, f\right)\right)$ in the setting of entropy solutions.

- Inequlities (3.5) are multi-valued; but, approximating $k$ from below and from above, it is enough to require that (3.5) holds in its less restrictive version, i.e., with $\left(\inf \beta_{(t, x)}(k)-\right.$ $\left.\varphi_{\nu(x)}(k)\right)^{-}$and with $\left(\sup \beta_{(t, x)}(k)-\varphi_{\nu(x)}(k)\right)^{+}$, respectively, in the right-hand side.

- Definition (i) is a straightforward interpretation of the formal $B C$, encoded by $\beta_{(t, x)}$, as the effective $B C$ given by its projection $\tilde{\beta}_{(t, x)}$ : recall that $\widetilde{D}_{(t, x)}^{0}$ is the domain of $\tilde{\beta}_{(t, x)}$.

- Definition (ii) of entropy solutions encrypts, in a rather direct way, the dissipative nature of the boundary condition expressed by $\tilde{\beta}_{(t, x)}$. Combination of items (i) and (ii) leads to an immediate proof of uniqueness, comparison and $L^{1}$ contraction for entropy solutions.

- Explicit use of boundary traces in Definitions (i),(ii) makes it delicate to establish existence. Indeed, one of important features of a definition should be the stability of the notion of entropy solution under $L_{l o c}^{1}$ convergence in $Q_{T}$. Existence arguments for Definitions (i),(ii) were devised in [36,6] but they are quite restrictive (namely, they require strong compactness of boundary traces on $\Sigma$, which is not implied by a mere $L_{l o c}^{1}\left(Q_{T}\right)$ convergence).

- "Traceless" definitions (iv) and (v) by global entropy inequalities (cf. [7] for a different version of Definition (iv)) are clearly stable under $L_{l o c}^{1}$ convergence.

\footnotetext{
${ }^{10}$ Truncation by $C_{k}$ is needed in order that the right-hand side be finite. Indeed, recall that we have extended $\beta_{(t, x)}$ to an $\overline{\mathbb{R}}$-valued graph.

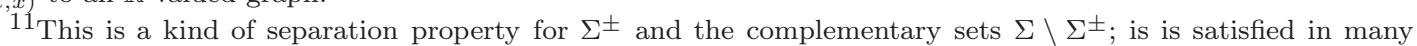
practical situations, but it fails e.g. in $\beta_{(t, x)}=\left\{u^{D}(t, x)\right\} \times \mathbb{R}$ (the Dirichlet case) with $u^{D}$ that is the characteristic function of a Cantor set of positive measure.
} 
- Definition (v) is reminiscent of those of Carrillo [15], Ammar, Carrillo and Wittbold [2]. Yet in full generality, (v) cannot be used e.g. when $\Sigma^{ \pm}$have a fractal nature. Definition (iv) is a way to bypass the subtlety of the simultaneous choice of $k$ and $\xi$ imposed in [15]; the idea is to incorporate a remainder term that vanishes, on parts of the boundary, for particular choices of $k$. Approach similar to (iv) was used by Vovelle [40], with a simpler choice of the remainder term suitable for inhomogeneous Dirichlet boundary condition.

- Finally, definition (iii) provides a link between (i)-(ii) and (iv)-(v): it uses both traces and the " $D^{ \pm}$vocabulary". This definition can be put in correspondence with the pointwise interpretation by Rouvre and Gagneux [35] of the Carrillo boundary condition.

The following proposition defines entropy sub- and super-solutions of problem $\left(H_{\varphi, \beta}\left(u_{0}, f\right)\right)$.

Proposition 3.5 (Definition of entropy sub- and super-solutions). Let $u \in L^{\infty}\left(Q_{T}\right)$.

If any of the below items (i)-(iv) is satisfied, $u$ is called an entropy sub-solution of problem $\left(H_{\varphi, \beta}\left(u_{0}, f\right)\right)$. Indeed, the assertions (i)-(iv) are equivalent:

(i) The function $u$ verifies the entropy inequalities (3.1) with the sign "plus" and $\xi \in \mathcal{D}([0, T) \times$ $\Omega), \xi \geq 0$, moreover, for $\mathcal{H}^{N}$-a.e. $(t, x) \in \Sigma$, the strong trace $\gamma V \varphi_{\nu(x)}$ u lies in $V \varphi_{\nu(x)} \widetilde{D}_{(t, x)}^{-}$.

(ii) The function $u$ verifies the entropy inequalities (3.1) with the sign "plus" with $\xi \in$ $\mathcal{D}([0, T) \times \Omega), \xi \geq 0$, moreover, for $\mathcal{H}^{N}$-a.e. $(t, x) \in \Sigma$, the strong trace $\gamma V \varphi_{\nu(x)} u$ verifies

$$
\forall k \in \widetilde{D}_{(t, x)}^{+} ; \quad Q_{\nu(x)}^{+}\left(\gamma V \varphi_{\nu(x)} u, V \varphi_{\nu(x)} k\right) \geq 0 .
$$

(iii) Item (ii) holds with $k \in D_{(t, x)}^{+}$(in the place of $k \in \widetilde{D}_{(t, x)}^{+}$) in (3.8).

(vi) The function $u$ verifies the up-to-the-boundary entropy inequalities with remainder term

$$
\forall k \in \mathbb{R} \forall \xi \in \mathcal{D}([0, T) \times \bar{\Omega}), \xi \geq 0,
$$

$$
\begin{aligned}
& \int_{0}^{T} \int_{\Omega}\left(-(u-k)^{+} \xi_{t}-q^{+}(u, k) \cdot \nabla \xi\right)-\int_{\Omega}\left(u_{0}-k\right)^{+} \xi(0, \cdot) \\
& \quad \leq \int_{0}^{T} \int_{\Omega} \operatorname{sign}^{+}(u-k) f \xi+\iint_{\Sigma} C_{k} \wedge\left(\beta_{(t, x)}(k)-\varphi_{\nu(x)}(k)\right)^{-} \xi(t, x) .
\end{aligned}
$$

Here, $C_{k}$ is a constant that depends on $\|u\|_{\infty}$ and on $k$.

Further, exchange the signs "plus" and "minus" in the above properties: they remain equivalent, and if any of them is satisfied, $u$ is called an entropy super-solution of problem $\left(H_{\varphi, \beta}\left(u_{0}, f\right)\right)$.

The proof of Proposition 3.5 uses the same tools as the one of Proposition 3.3 given below; we omit the details.

Remark 3.6. A function $u$ is an entropy solution of problem $\left(H_{\varphi, \beta}\left(u_{0}, f\right)\right)$ if and only if it is both entropy sub- and super-solution of the problem.

3.2. Proof of the equivalence of different definitions. Before turning to the proof, let us state the key technical lemma that allows for a use of strong traces defined in the way of Section 1.3.

Lemma 3.7. There exists a sequence $\left(\xi_{n}\right)_{n}$ of Lipschitz functions on $\bar{\Omega}$ such that $0 \leq \xi_{n} \leq 1$, $\left.\xi_{n}\right|_{\partial \Omega}=1, \xi_{n} \rightarrow 0$ on $\Omega$ as $n \rightarrow \infty$, and for all $\xi \in \mathcal{D}([0, T) \times \bar{\Omega})$, for all $k \in \mathbb{R}$ there holds

$$
\begin{aligned}
& \lim _{n \rightarrow \infty}\left(\int_{0}^{T} \int_{\Omega}(\right.\left.\left.-(u-k)^{ \pm}\left(\xi \xi_{n}\right)_{t}-q^{ \pm}(u, k) \cdot \nabla\left(\xi \xi_{n}\right)\right)-\int_{\Omega}\left(u_{0}-k\right)^{ \pm}\left(\xi \xi_{n}\right)(0, \cdot)\right) \\
&=-\lim _{n \rightarrow \infty} \int_{0}^{T} \int_{\Omega} \xi q^{ \pm}(u, k) \cdot \nabla \xi_{n}=-\iint_{\Sigma} \xi Q_{\nu(x)}^{ \pm}\left(\gamma V_{\varphi_{\nu(x)}} u, V_{\varphi_{\nu(x)}} k\right)
\end{aligned}
$$


and

$$
\lim _{n \rightarrow \infty} \int_{0}^{T} \int_{\Omega} q^{ \pm}(u, k) \cdot \nabla\left(\xi\left(1-\xi_{n}\right)\right)=\int_{0}^{T} \int_{\Omega} q^{ \pm}(u, k) \cdot \nabla \xi-\iint_{\Sigma} \xi Q_{\nu(x)}^{ \pm}\left(\gamma V_{\varphi_{\nu(x)}} u, V_{\varphi_{\nu(x)}} k\right) .
$$

Proof. For $n \in \mathbb{N}$, the function $\xi_{n}$ is defined almost explicitly. Firstly, a partition of unity $\left(\chi^{i}\right)_{i=0}^{M}$ on $\bar{\Omega}$ is used such that supp $\chi^{0} \subset \Omega$ and for $i=1 . . M$, supp $\chi^{i} \subset U^{i}$ where $U^{i}$ is an open set of the kind considered in Section 1.3. Then for each $i=1 . . M$, in the local coordinates of $U_{i}$ as described in Section 1.3 we take the function

$$
\pi_{n}^{i}\left(x_{0}, x^{\prime}\right):=n\left(\frac{1}{n}-\left(x_{0}-g^{i}\left(x^{\prime}\right)\right)\right)^{+}
$$

(the function $g^{i}$ being associated with the neighbourhood $U^{i}$ ). Then we assign

$$
\xi_{n}:=\sum_{i=1}^{M} \chi^{i} \pi_{n}^{i}
$$

Clearly, it only remains to justify (3.10) and (3.11).

Notice that $\nabla \xi_{n}=\sum_{i=1}^{M} \nabla \chi^{i} \pi_{n}^{i}+\sum_{i=1}^{M} \chi^{i} \nabla \pi_{n}^{i}$ and the first term in the right-hand side vanishes as $n \rightarrow \infty$, while the second one permits to make appeal to the strong normal traces of $V_{\varphi_{\nu(x)}} u$. Indeed, by construction $\nabla \pi_{n}^{i}(\cdot)$ is aligned with the field of normals $\nu(\cdot)$ lifted inside $U^{i}$; it is supported on $\left\{0<y=x_{0}-g^{i}\left(x^{\prime}\right)<\frac{1}{n}\right\}$ and its absolute value is $n \sqrt{1+\left|\nabla g^{i}\left(x^{\prime}\right)\right|^{2}}$. The limit of the expression

$$
\begin{aligned}
& \int_{0}^{T} \int_{U^{i}} \xi \chi^{i} q^{ \pm}(u, k) \cdot \nabla \pi_{n}^{i} \\
& \quad \equiv n \int_{0}^{\frac{1}{n}}\left(\int_{0}^{T} \int_{W^{i}} \xi \chi^{i} q^{ \pm}\left(u\left(t, y+g^{i}\left(x^{\prime}\right), x ;\right), k\right) \cdot \nu\left(x_{0}, x^{\prime}\right)\left(\sqrt{1+\left|\nabla g^{i}\left(x^{\prime}\right)\right|^{2}} d t d x\right)\right) d y
\end{aligned}
$$

(here $W^{i}$ is a boundary neighbourhood corresponding to $U^{i}$, see Section 1.3) exists and equals

$$
\iint_{\Sigma} \xi \chi^{i} Q_{\nu(x)}^{ \pm}\left(\gamma V_{\varphi_{\nu(x)}} u, V_{\varphi_{\nu(x)}} k\right)
$$

according to Section 1.3 and because $\left(\sqrt{1+\left|\nabla g^{i}\left(x^{\prime}\right)\right|^{2}} d t d x\right)$ is precisely the surface measure on the boundary $\Sigma$. Then by a straightforward passage to the limit, both (3.10) and (3.11) hold.

Proof of Proposition 3.3. Throughout the proof, we use the following notation. If a point $(t, x) \in$ $\Sigma$ is fixed, we set $\tilde{V}:=\gamma V \varphi_{\nu(x)} u$, then pick (arbitrarily) $\tilde{u} \in\left[V \varphi_{\nu(x)}\right]^{-1}(\tilde{V})$. We also use the sequence $\left(\xi_{n}\right)_{n}$ of Lemma 3.7 .

Notice that all the definitions contain entropy inequalities (3.1). We concentrate on the equivalence of the complementary properties related to the boundary condition.

$\underline{(\mathrm{i}) \Rightarrow(\mathrm{ii})}$ The claim is straightforward, by the definition (1.10) of $Q_{\nu(x)}^{ \pm}$and the monotonicity of the graph of $\left.\varphi_{\nu(x)}\right|_{\widetilde{D}_{(t, x)}^{0}}$.

(ii) $\Rightarrow$ (i) This implication is a consequence of the maximality of the graph $\tilde{\beta}_{(t, x)}$ as a monotone

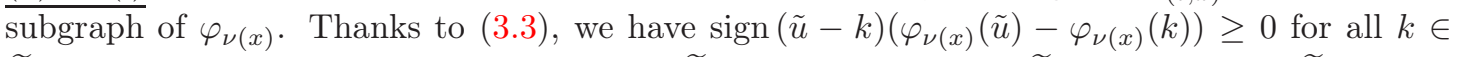
$\widetilde{D}_{(t, x)}^{0}$; thus, $\varphi_{\nu(x)}$ is monotone not only on $\widetilde{D}_{(t, x)}^{0}$ but also on $\tilde{u} \cup \widetilde{D}_{(t, x)}^{0}$. Thus $\tilde{u} \in \widetilde{D}_{(t, x)}^{0}$ and $\tilde{V} \in V \varphi_{\nu(x)} \widetilde{D}_{(t, x)}^{0}$, which proves (i).

(i) $\Leftrightarrow$ (iii) This equivalence follows from Proposition 2.5(i). 
(i) $\Rightarrow$ (iv) As a preliminary step, we assess the following property (see [36, 6]): for all $k \in \mathbb{R}$, for all $\xi \in \mathcal{D}([0, T) \times \bar{\Omega})^{+}$

$$
\begin{aligned}
\int_{0}^{T} \int_{\Omega}\left(-(u-k)^{ \pm} \xi_{t}-\right. & \left.q^{ \pm}(u, k) \cdot \nabla \xi\right)-\int_{\Omega}\left(u_{0}-k\right)^{ \pm} \xi(0, \cdot) \\
& \leq-\iint_{\Sigma} Q_{\nu(x)}^{ \pm}\left(\gamma V \varphi_{\nu(x)} u, V \varphi_{\nu(x)} k\right) \xi(t, x) .
\end{aligned}
$$

Indeed, taking (by approximation) for the test function in (3.1) a nonnegative function $\xi \in$ $\mathcal{D}([0, T) \times \bar{\Omega})$ multiplied by the truncation $\left(1-\xi_{n}\right)$, we get (3.12) from (3.11) of Lemma 3.7.

It remains to justify using the information that $\tilde{u} \in \widetilde{D}_{(t, x)}^{0}$, that

$$
-Q_{\nu(x)}^{ \pm}\left(\gamma V_{\varphi_{\nu(x)}} u, V_{\varphi_{\nu(x)}} k\right) \equiv-q_{\nu(x)}^{ \pm}(\tilde{u}, k) \leq C_{k} \wedge\left(\beta_{(t, x)}(k)-\varphi_{\nu(x)}(k)\right)^{\mp} .
$$

The upper bound of the left-hand side of (3.13) by $C_{k}:=2 \max \left\{|\varphi(z)||| z \mid \leq k+\|u\|_{\infty}\right\}$ is evident. Further, if $k \in D_{(t, x)}^{ \pm}$, then we already know that (i) implies (3.4), which gives

$$
-Q_{\nu(x)}^{ \pm}\left(\gamma V_{\varphi_{\nu(x)}} u, V_{\varphi_{\nu(x)}} k\right) \leq 0 \leq\left(\beta_{(t, x)}(k)-\varphi_{\nu(x)}(k)\right)^{\mp},
$$

proving (3.13) for this case. Let us study the remaining values of $k$.

For the sake of being definite, let us consider $k \in D_{(t, x)}^{-}, k<\tilde{u}$; then the goal is to estimate $-q_{\nu(x)}^{+}(\tilde{u}, k)$ from above by $R_{k}:=\left(\beta_{(t, x)}(k)-\varphi_{\nu(x)}(k)\right)^{-}$. Consider the four possible cases.

- If $\varphi_{\nu(x)}(\tilde{u}) \geq \varphi_{\nu(x)}(k)$, there is nothing to prove because $-q_{\nu(x)}^{+}(\tilde{u}, k) \leq 0 \leq R_{k}$.

- If $\beta_{(t, x)}(k) \leq \varphi_{\nu(x)}(\tilde{u})<\varphi_{\nu(x)}(k)$, then

$$
-q_{\nu(x)}^{+}(\tilde{u}, k) \equiv \varphi_{\nu(x)}(k)-\varphi_{\nu(x)}(\tilde{u}) \leq \varphi_{\nu(x)}(k)-\beta_{(t, x)}(k)=\left(\beta_{(t, x)}(k)-\varphi_{\nu(x)}(k)\right)^{-}=R_{k} .
$$

- If $\tilde{u} \in D_{(t, x)}^{-}$then $\varphi_{\nu(x)}(\tilde{u}) \geq \beta_{(t, x)}(\tilde{u})$ and from the monotonicity of $\beta_{(t, x)}$, we do have

$$
-q_{\nu(x)}^{+}(\tilde{u}, k) \equiv \varphi_{\nu(x)}(k)-\varphi_{\nu(x)}(\tilde{u}) \leq \varphi_{\nu(x)}(k)-\beta_{(t, x)}(\tilde{u}) \leq \varphi_{\nu(x)}(k)-\beta_{(t, x)}(k)=R_{k} .
$$

- It remains the case $\tilde{u} \in D_{(t, x)}^{+}, k \in D_{(t, x)}^{-}$, and $\varphi_{\nu(x)}(\tilde{u})<\beta_{(t, x)}(k)<\varphi_{\nu(x)}(k)$; let us show that this is impossible. Indeed, in this case there exists $k^{\prime} \in(k, \tilde{u}]$ that belongs to $D_{(t, x)}^{0}$. Then $k^{\prime} \in \widetilde{D}_{(t, x)}^{0}$ according to the definition of this graph. Yet also $\tilde{u} \in \widetilde{D}_{(t, x)}^{0}$; by the definition of $D_{(t, x)}^{0}$ and the monotonicity of $\left.\varphi_{\nu(x)}\right|_{\widetilde{D}_{(t, x)}^{0}}$, we infer

$$
\beta_{(t, x)}\left(k^{\prime}\right) \ni \varphi_{\nu(x)}\left(k^{\prime}\right) \leq \varphi_{\nu(x)}(\tilde{u})<\beta_{(t, x)}(k) .
$$

This contradicts the monotonicity of $\beta_{(t, x)}$ because $k<k^{\prime}$.

(iv) $\Rightarrow$ (iii) It is enough to justify inequalities (3.4). We work with mollifying sequences $\left(\xi^{\alpha}\right)_{\alpha}$ on $\Sigma$ (extended smoothly inside $\Omega$ ) that are supported in an $\alpha$-neighbourhood of some $\sigma \in \Sigma$; as $\alpha \rightarrow 0, \xi^{\alpha}$ concentrates to the Dirac measure supported at $\sigma$.

Fix $k \in \mathbb{R}$ and consider e.g. $\sigma=(t, x) \in \Sigma^{+}(k)$. Almost every point of $\Sigma^{+}(k)$ is its point of density (see, e.g., [18]), which means in particular that, for $\mathcal{H}^{N}$-a.e. $\sigma \in \Sigma^{+}(k)$,

$$
\lim _{\alpha \rightarrow 0} \iint_{\Sigma} C_{k} \wedge\left(\beta_{(t, x)}(k)-\varphi_{\nu(x)}(k)\right)^{-} \xi^{\alpha}(t, x)=0 .
$$

Indeed, the integrand in the right-hand side is bounded by $C_{k}$ and by the definition (3.2), it is zero for $(t, x) \in \Sigma^{+}(k)$. 
Now we generate inequalities (3.4) by taking the test functions $\xi^{\alpha} \xi_{n}$ (with $\left(\xi_{n}\right)_{n}$ constructed in Lemma 3.7). Using consequently (3.10), (3.5) and (3.15), we infer

$$
\begin{gathered}
-\left.Q_{\nu(x)}^{+}\left(\gamma V_{\varphi_{\nu(x)}} u, V_{\varphi_{\nu(x)}} k\right)\right|_{(t, x)=\sigma}=-\lim _{\alpha \rightarrow 0} \iint_{\Sigma} \xi^{\alpha} Q_{\nu(x)}^{+}\left(\gamma V_{\varphi_{\nu(x)}} u, V_{\varphi_{\nu(x)}} k\right) \\
=\lim _{\alpha \rightarrow 0} \lim _{n \rightarrow \infty}\left(\int_{0}^{T} \int_{\Omega}\left(-(u-k)^{ \pm}\left(\xi^{\alpha} \xi_{n}\right)_{t}-q^{+}(u, k) \cdot \nabla\left(\xi^{\alpha} \xi_{n}\right)\right)-\int_{\Omega}\left(u_{0}-k\right)^{ \pm}\left(\xi^{\alpha} \xi_{n}\right)(0, \cdot)\right) \\
\leq \lim _{\alpha \rightarrow 0} \iint_{\Sigma} C_{k} \wedge\left(\beta_{(t, x)}(k)-\varphi_{\nu(x)}(k)\right)^{-} \xi^{\alpha}(t, x)=0 .
\end{gathered}
$$

Similarly, the case $\sigma \in \Sigma^{-}(k)$ leads to the inequality $-\left.Q_{\nu(x)}^{-}\left(\gamma V_{\varphi_{\nu(x)}} u, V_{\varphi_{\nu(x)}} k\right)\right|_{(t, x)=\sigma} \leq 0$. $\underline{(\mathrm{iv}) \Rightarrow(\mathrm{v})}$ Inequalities (3.7) are immediate from (3.5).

$(\mathrm{v}) \&(3.6) \Rightarrow(\mathrm{i})$ Actually, we rather prove (iii). Under the assumption that there exists a mol$\overline{\text { lifying sequence }}\left(\xi^{\alpha}\right)_{\alpha}$ on $\Sigma$ that concentrates at $\sigma \in \Sigma^{ \pm}(k)$ and, moreover, that is identically zero on $\Sigma \backslash \Sigma^{ \pm}(k)$, we can repeat the proof of the above implication "(iv) $\Rightarrow$ (iii)". A sufficient condition is the density of $\mathcal{D}\left(\Sigma^{ \pm}(k)\right)$ in $L^{1}\left(\Sigma^{ \pm}(k)\right)$. Moreover, this assumption is needed only for a countable dense set of values of $k$ : indeed, the proof of the implication "(iii) $\Rightarrow$ (i)" can be rewritten so that it use only a dense subset of values of $k$ satisfying (3.4). Thus, (3.6) is enough to derive the trace condition in (i).

\section{UNIQUENESS, COMPARISON, CONTINUOUS DEPENDENCE}

Following the ideas of $[21,11,25,5]$, introduce the "uniqueness condition"

$$
\text { either } \Omega \text { is bounded, or } N=1 \text {, or } \varphi \text { is locally Hölder continuous of order } 1-\frac{1}{N} \text {. }
$$

In the classical case of a locally Lipschitz continuous flux $\varphi$ this assumption holds automatically.

The following result contains uniqueness of an entropy solution for problem $\left(H_{\varphi, \beta}\left(u_{0}, f\right)\right), L^{1}$ contraction and comparison property with respect to the initial datum $u_{0}$ and the source term $f$, and a comparison and stability property with respect to the choice of $\beta_{(t, x)}(\cdot)$.

Theorem 4.1. Assume (4.1). Let $u^{1}$ be an entropy sub-solution for Problem $\left(H_{\varphi, \beta^{1}}\left(u_{0}^{1}, f^{1}\right)\right)$; let $u^{2}$ be an entropy super-solution for Problem $\left(H_{\varphi, \beta^{2}}\left(u_{0}^{2}, f^{2}\right)\right)$. Then for a.e. $s \in(0, T)$,

$$
\int_{\Omega}\left(u^{1}-u^{2}\right)^{+}(s) \leq \int_{\Omega}\left(u_{0}^{1}-u_{0}^{2}\right)^{+}+\int_{0}^{s} \int_{\Omega} \operatorname{sign}^{+}\left(u^{1}-u^{2}\right)\left(f^{1}-f^{2}\right)+\int_{0}^{t} \int_{\partial \Omega} d_{x}^{-}\left(\tilde{\beta}_{(t, x)}^{1}, \tilde{\beta}_{(t, x)}^{2}\right) .
$$

In particular, if $u_{0}^{1} \leq u_{0}^{2}$ a.e. on $\Omega, f^{1} \leq f^{2}$ a.e. on $Q_{T}$ and if $\beta_{(t, x)}^{1} \succeq_{x} \beta_{(t, x)}^{2} \mathcal{H}^{N}-$ a.e. on $\Sigma$, then $u^{1} \leq u^{2}$ a.e. on $Q_{T}$. In particular, there exists at most one entropy solution to $\left(H_{\varphi, \beta}\left(u_{0}, f\right)\right)$.

Note that, whenever $\varphi$ is locally Lipschitz continuous, we can localize the contraction property using the finite speed of propagation, following Kruzhkov [20].

Proof. Consider the case of a bounded domain $\Omega$. We apply the Kruzhkov doubling of variables argument inside the domain to deduce the Kato inequality: for a.e. $s \in(0, T)$, for all $\xi \in \mathcal{D}(\Omega)$,

$$
\int_{\Omega}\left(u^{1}-u^{2}\right)^{+}(s) \xi-\int_{\Omega}\left(u_{0}^{1}-u_{0}^{2}\right)^{+} \xi(0, \cdot) \leq \int_{0}^{s} \int_{\Omega}\left(q^{+}\left(u^{1}, u^{2}\right) \cdot \nabla \xi+\operatorname{sign}^{+}\left(u^{1}-u^{2}\right)\left(f^{1}-f^{2}\right) \xi\right) .
$$

Now we take $\xi=1-\xi_{n}$ with $\left(\xi_{n}\right)_{n}$ constructed in Lemma 3.7, and let $n \rightarrow \infty$. Since there exists a strong normal boundary trace of $q^{+}\left(u_{1}, u_{2}\right)$ expressed in the way of Remark 1.1 in Section 1.3, 
we find the inequality

$$
\begin{array}{r}
\int_{\Omega}\left(u^{1}-u^{2}\right)^{+}(s) \leq \int_{\Omega}\left(u_{0}^{1}-u_{0}^{2}\right)^{+}+\int_{0}^{s} \int_{\Omega} \operatorname{sign}^{+}\left(u^{1}-u^{2}\right)\left(f^{1}-f^{2}\right) \\
\quad-\int_{0}^{s} \int_{\Omega} Q_{\nu(x)}^{+}\left(\gamma V \varphi_{\nu(x)}\left(u^{1}\right), \gamma V \varphi_{\nu(x)}\left(u^{2}\right)\right) .
\end{array}
$$

It remains to show that $-Q_{\nu(x)}^{+}\left(\gamma V \varphi_{\nu(x)}\left(u^{1}\right), \gamma V \varphi_{\nu(x)}\left(u^{2}\right)\right) \leq d_{x}^{-}\left(\tilde{\beta}_{(t, x)}^{1}, \tilde{\beta}_{(t, x)}^{2}\right)$ pointwise on $(0, T) \times$ $\partial \Omega$. This is true because whenever the term on the left is non-zero, we have

$$
-Q_{\nu(x)}^{+}\left(\gamma V \varphi_{\nu(x)}\left(u^{1}\right), \gamma V \varphi_{\nu(x)}\left(u^{2}\right)\right)=-\left(\varphi_{\nu(x)}\left(\tilde{u}^{1}\right)-\varphi_{\nu(x)}\left(\tilde{u}^{2}\right)\right)
$$

with some $\tilde{u}^{1}>\tilde{u}^{2}$ such that

$$
V \varphi_{\nu(x)}\left(\tilde{u}^{1}\right)=\gamma V \varphi_{\nu(x)}\left(u^{1}\right) \in V \varphi_{\nu(x)} \widetilde{D}^{-}(t, x) \text { and } V \varphi_{\nu(x)}\left(\tilde{u}^{2}\right)=\gamma V \varphi_{\nu(x)}\left(u^{2}\right) \in V \varphi_{\nu(x)} \widetilde{D}^{+}(t, x)
$$

(here we have used the trace properties of entropy sub- and super-solutions, see Proposition 3.5(i)). Thus $\varphi_{\nu(x)}\left(\tilde{u}^{1}\right) \geq \widetilde{\mathcal{B}^{1}}{ }_{(t, x)}\left(\tilde{u}^{1}\right), \quad \varphi_{\nu(x)}\left(\tilde{u}^{2}\right) \leq \widetilde{\mathcal{B}^{2}}{ }_{(t, x)}\left(\tilde{u}^{2}\right)$, so that the right-hand side of (4.4) fulfills

$$
-\left(\varphi_{\nu(x)}\left(\tilde{u}^{1}\right)-\varphi_{\nu(x)}\left(\tilde{u}^{2}\right)\right) \leq-\left(\widetilde{\mathcal{B}}_{(t, x)}\left(\tilde{u}^{1}\right)-{\widetilde{\mathcal{B}^{2}}}_{(t, x)}\left(\tilde{u}^{2}\right)\right) \leq d_{x}^{-}\left(\widetilde{\mathcal{B}}_{(t, x)}, \widetilde{\mathcal{B}}_{(t, x)}\right),
$$

where we have used the definition of $d_{x}^{-}$and the fact that $\tilde{u}^{1}>\tilde{u}^{2}$.

For the case when $\Omega$ is unbounded, in the same way we get the up-to-the boundary Kato inequality, i.e., inequality (4.3) with a test function $\xi \in \mathcal{D}(\bar{\Omega})$. Assuming either that $N=1$, or that $N \geq 2$ and $\varphi$ is locally Hölder continuous of order $1-\frac{1}{N}$ we use the techniques known for scalar conservation laws with infinite speed of propagation (see, e.g., [21, 11, 5]), and eventually deduce (4.2).

\section{EXISTENCE: A FORMAL PROOF}

In this section, we establish existence on an entropy solution but we take for granted that the formal BC, encrypted by the graphs $\beta_{(t, x)}$, should be replaced by the boundary condition expressed with the help of their projections $\widetilde{\mathcal{B}}_{(t, x)}=\widetilde{\mathcal{P}}_{x} \beta_{(t, x)}$. Section 6 contains a longer but more convincing discussion of the problem of existence and convergence of approximations.

For general graphs $\beta$ satisfying the measurability assumption (1.1), we cannot hope for existence of a bounded solution (it is enough to consider, e.g., the situation where unbounded Dirichlet data are imposed: in this case, one needs the notion of a renormalized solution, as used by Porretta, Vovelle [34] and by Ammar, Carrillo and Wittbold [2]). We control the $L^{\infty}$ norm of solutions or approximate solutions by assuming existence of a rich enough family of simple (constant in space) sub- and super-solutions to the problem. Namely, we require that one of the two following assumptions be fulfilled: either

$$
\begin{aligned}
& f=0 \text {, and there exist }\left(A_{m}^{-}\right)_{m \in \mathbb{N}},\left(A_{m}^{+}\right)_{m \in \mathbb{N}} \subset \mathbb{R}^{ \pm} \text {such that } A_{m}^{ \pm} \rightarrow \pm \infty \\
& \text { as } m \rightarrow \infty \text { and for } \mathcal{H}^{N-\text { a.e. }}(t, x) \in \Sigma \text {, for all } m \in \mathbb{N} \quad A_{m}^{ \pm} \in \widetilde{D}_{(t, x)}^{ \pm}
\end{aligned}
$$

or

(5.2) the measures of the sets $\mathcal{A}^{ \pm}:=\left\{k \in \mathbb{R}^{ \pm} \mid k \in \widetilde{D}_{(t, x)}^{ \pm}\right.$for $\mathcal{H}^{N}$-a.e. $\left.(t, x) \in \Sigma\right\}$ are infinite.

Note that (5.2) is ensured by the following:

$$
\exists A \text { for } \mathcal{H}^{N} \text {-a.e. }(t, x) \in \Sigma \quad(-\infty,-A] \subset \widetilde{D}_{(t, x)}^{-} \text {and }[A,+\infty) \subset \widetilde{D}_{(t, x)}^{+} \text {. }
$$


Remark 5.1. Given a formal BC graph $\beta$, it is not immediate to check whether (5.3), (5.1), or (5.2) hold. Let us give sufficient conditions.

Firstly, by definition we have $D_{(t, x)}^{ \pm} \subset \widetilde{D}_{(t, x)}^{ \pm}$, where $D_{(t, x)}^{+}$, resp. $D_{(t, x)}^{-}$is the overshoot (resp., the undershoot) set defined in Section $2 ; D_{(t, x)}^{ \pm}$are computed directly from the relative positions of the graphs $\beta_{(t, x)}$ and $\varphi_{\nu(x)}$. Thus replacing $\widetilde{D}_{(t, x)}^{ \pm}$by $D_{(t, x)}^{ \pm}$in each of the assumptions (5.3), (5.1), or (5.2), we get stronger but easier-to-check restrictions (cf. [6, 7]).

Secondly, if there exists $C>0$ such that for all $x \in \partial \Omega$ each of the functions $\left.\varphi_{\nu(x)}\right|_{(-\infty, C]}$ and $\left.\varphi_{\nu(x)}\right|_{[C,+\infty)}$ is either non-decreasing or non-increasing, then it is easily checked that assumption (5.3) (and thus also (5.2)) holds.

Assume in addition that the limit flux $\varphi$ is genuinely nonlinear in the sense (5.4) $\forall \Xi \in \mathbb{R}^{N+1} \backslash\{0\} \forall c \in \mathbb{R}$ the Lebesque measure of the set $\{z \mid \Xi \cdot(z, \varphi(z))=c\}$ is zero.

Notice that the latter assumption implies (1.6), in particular the singular mapping $V \varphi_{\nu(x)}$ can be taken to be $I d$ in this case.

The main result is the following theorem.

Theorem 5.2. Assume that $\varphi$ satisfies (4.1), (5.4). Let $u_{0} \in L^{\infty}(\Omega)$ and $\int_{0}^{T}\|f(t, \cdot)\|_{\infty} d t<\infty$. Assume that $\beta$ satisfies (1.1) and any of the assumptions (5.1), (5.2). Then there exists a unique entropy solution of problem $\left(H_{\varphi, \beta}\left(u_{0}, f\right)\right)$.

Proof. Uniqueness is contained in Theorem 4.1. For proving existence, we exploit the vanishing viscosity method in which we use directly the projected graphs $\widetilde{\mathcal{B}}_{(t, x)}=\widetilde{\mathcal{P}}_{x} \beta_{(t, x)}$. We apply two results that are justified in the sequel. Firstly, we construct approximate solutions $u^{\varepsilon}$ by the vanishing viscosity method, using Proposition 9.6 (see also Remark 9.7) of Appendix. Indeed, $k \mapsto \widetilde{\mathcal{B}}_{(t, x)}(k)=: b(t, x ; k)$ being a continuous function for fixed $(t, x) \in \Sigma$, from (1.1) we deduce that the map $b$ on $\Sigma \times \mathbb{R}$ is Carathéodory. Because Proposition 9.6 requires that $b$ be bounded, we pick some value $M>0$ depending on $\left\|u_{0}\right\|_{\infty}+\int_{0}^{T}\|f(t, \cdot)\|_{\infty} d t$ and on $\left(A_{m}^{ \pm}\right)_{m}$ or $\mathcal{A}^{ \pm}$in the assumptions ( $M$ is chosen as a priori bound of $\|u\|_{\infty}$, to be justified later). We proceed by truncating $\varphi$ and $\widetilde{\mathcal{B}}_{(t, x)}$ as follows: e.g., under assumption (5.1) we take $m$ such that $\left[A_{m}^{-}, A_{m}^{+}\right] \supset$ $[-M, M]$ with $M=\left\|u_{0}\right\|_{\infty}$ and take the convention that

$$
\begin{aligned}
& \varphi \text { is constant on }\left(-\infty, A_{m}^{-}\right] \text {and on }\left[A_{m}^{+},+\infty\right) \text { (equal to } \varphi\left(A_{m}^{ \pm}\right) \text {, respectively), } \\
& \widetilde{\mathcal{B}}_{(t, x)} \text { is constant on }\left(-\infty, A_{m}^{-}\right] \text {and on }\left[A_{m}^{+},+\infty\right) \text { (equal to } \widetilde{\mathcal{B}}_{(t, x)}\left(A_{m}^{ \pm}\right) \text {, respectively). }
\end{aligned}
$$

Therefore we get existence of vanishing viscosity approximations $\left(u^{\varepsilon}\right)_{\varepsilon}$ corresponding to the truncated graphs.

Let us stress the fact that, because $A_{m}^{ \pm} \in \widetilde{D}_{(t, x)}^{ \pm}$, truncation (5.5) does not change the fact that $\widetilde{\mathcal{B}}_{(t, x)}$ is a maximal monotone subgraph of $\varphi_{\nu(x)}$. For the same reason, the truncated graphs $\varphi$ and $\widetilde{\mathcal{B}}$ fulfill assumption $(5.1)$ with the same sequences $\left(A_{m}^{ \pm}\right)_{m}$; hence by Proposition 9.6(ii) the solutions obey an $L^{\infty}$ estimate that does not depend on the truncation level chosen in (5.5).

Now we can exploit Theorem 6.2 stated and proved in Section 6.1. Its assumptions (6.1)-(6.3) are fulfilled: indeed, notice that we have required the genuine nonlinearity property (5.4) that implies compactness (see, e.g., $[29,32]$ ) and that $\widetilde{\mathcal{B}}$ are bounded by $\max _{\left[A_{m}^{-}, A_{m}^{+}\right]}|\varphi|$ due to the truncation convention (5.5). We deduce existence of an entropy solution $u$ to the truncated problem $\left(H_{\varphi, \beta}\left(u_{0}, f\right)\right)$. Yet we have also ensured that $\|u\|_{\infty} \leq M$, therefore the constructed solution $u$ also solves the original problem $\left(H_{\varphi, \beta}\left(u_{0}, f\right)\right.$ ) (before the truncation (5.5)). This ends the proof. 


\section{JUSTIFICATION OF THE EFFECTIVE BOUNDARY CONDITION}

The goal of this section is to provide evidence in favor of the interpretation $(1.12),\left(A_{\tilde{\beta}}\right)$ of the effective boundary condition. As it was already stated in the introduction, a natural way to justify a notion of solution is to see problem $\left(H_{\varphi, \beta}\left(u_{0}, f\right)\right)$ as the limit of a family of problems for which the notion of solution is unambiguous: one derives the solution notion from passage-tothe-limit arguments. In this section, we do it in two complementary ways, following the general idea of our previous works ([6] and [7], respectively).

Firstly, in Section 6.1 we rely on the classical notion of weak solution to parabolic problems with additional viscosity term, vanishing at the limit. The entropy formulation of $\left(H_{\varphi, \beta}\left(u_{0}, f\right)\right)$ is obtained as a singular limit formulation: indeed, the limit problem looses its parabolic character. Unfortunately, for a practical application of this technique we will need several restrictions on the behaviour of $\beta$ and $\varphi$. To separate the technical details from the key idea of the proof, we assume, without comment, that approximate solutions possess uniform bounds and a strong compactification property. Notice that the techniques of Section 6.1 are very different from the ones of the preceding works $[36,6]$, where we also needed the difficult to ensure compactification assumptions on the sequence of approximate solutions on the boundary.

Remark 6.1. Although the arguments of $[36,6]$ are less general, they have the advantage of showing quite explicitly how the projected graph $\tilde{\beta}$ (in its characterization [6, formula (4)], equivalent to the characterization of Proposition 2.5(ii)) appears from $\beta$.

In a sense, with [6, formula (4)] one can "observe" the formation of the boundary layer (see [36] for details). The arguments we use in this paper are more indirect; they lead to the characterization of Proposition 2.5(i), via the formulation (3.5).

Secondly, in Section 6.2 we consider approximations of $\left(H_{\varphi, \beta}\left(u_{0}, f\right)\right)$ by purely hyperbolic problems of the same type (but with possibly different data and non-linearities) and exploit the stability and comparison principle of Theorem 4.1 in order to extend the entropy formulation "by heredity". This allows, e.g., to concentrate on the case of smooth and/or compactly supported initial data, that may be useful in the context of a locally Lipschitz flux $\varphi$ (cf. Section 7.1). For the Dirichlet or obstacle condition, we can approximate the boundary data either pointwise or using the Lusin theorem. For a less evident application, one can approximate a general graph $\beta$ by a bi-monotone sequence of graphs $\beta^{m, n}$ satisfying the assumptions of the previous section (by bi-monotonicity, it is meant that $\beta^{\delta, \lambda}$ decreases as $\delta \downarrow 0$ and increases as $\left.\lambda \downarrow 0\right)$. In this way we can justify the use of the projected graph $\widetilde{\beta}$ for the homogeneous Neumann boundary condition (whereas the justification in the way of Section 6.1 does not work in this case); see [7].

6.1. Convergence of the vanishing viscosity approximation. Let us provide a basic convergence argument for the vanishing viscosity approximation (without any additional regularization or approximation of data and nonlinearities).

We make the following a priori assumptions on data and nonlinearities of problem (1.3):

For all $\varepsilon \in(0,1)$ there exists a weak solution ${ }^{12} u^{\varepsilon} \in L^{2}\left(0, T ; H_{l o c}^{1}(\Omega)\right)$ of $(1.3)$, moreover, the family $\left(\sqrt{\varepsilon} \nabla u^{\varepsilon}\right)_{\varepsilon}$ is bounded in $L_{l o c}^{2}([0, T] \times \Omega)$;

There exists $u \in L^{\infty}\left(Q_{T}\right)$ and a sequence $\varepsilon_{m}$ decreasing to zero as $m \rightarrow \infty$ such that $u^{\varepsilon_{m}} \rightarrow u$ in $L_{l o c}^{1}([0, T] \times \Omega)$ as $m \rightarrow \infty$.

There exists $G \in L_{l o c}^{1}([0, T] \times \partial \Omega)$ such that $\left|b^{\varepsilon}(t, x)\right| \leq G(t, x)$ for $\mathcal{H}^{N}$-a.e. $(t, x) \in \Sigma$, uniformly in $\varepsilon \in(0,1)$,

\footnotetext{
12 see Appendix for a precise definition of a weak solution
} 
where $b^{\varepsilon}(t, x) \in \beta_{(t, x)}\left(u^{\varepsilon}(t, x)\right)$ is the value realized in the multi-valued boundary condition of (1.3) (namely, $b^{\varepsilon}(t, x):=\gamma_{w}\left(\varphi\left(u^{\varepsilon}\right)-\varepsilon \nabla u^{\varepsilon}\right) \cdot \nu(x)$, the right-hand side having the meaning of the weak normal boundary trace of the divergence-measure field $\left(\varphi\left(u^{\varepsilon}\right)-\varepsilon \nabla u^{\varepsilon}\right)$, see [16]). Writing $\beta_{(t, x)}\left(u^{\varepsilon}(t, x)\right)$, we use without further mention the restriction $\left.u^{\varepsilon}\right|_{\Sigma}$ of $u^{\varepsilon}$ on the boundary, understood in the sense of traces of Sobolev functions.

Theorem 6.2. Assume that $u_{0}, f$ and $\varphi, \beta$ are such that (6.1),(6.2) and (6.3) hold. Then $u$ is an entropy solution of problem $\left(H_{\varphi, \beta}\left(u_{0}, f\right)\right)$.

Remark 6.3. In practice, (6.2) can be fulfilled as a compactness property. In this case, let us suppose that the uniqueness condition (4.1) of Theorem 4.1 holds. Then from the uniqueness of the accumulation point $u$ we deduce that the whole family $u^{\varepsilon}$ converges, as $\varepsilon \rightarrow 0$, to the entropy solution of $\left(H_{\varphi, \beta}\left(u_{0}, f\right)\right)$.

Proof. It is classical (see e.g. Carrillo [15]) to deduce from the weak formulation of (1.3) the Kruzhkov entropy inequalities $(3.1)$ with $\mathcal{D}([0, T) \times \Omega)$ test functions (i.e., entropy formulation inside the domain). One readily passes to the limit in this entropy formulation using the property (6.2) and the uniform $L_{l o c}^{2}$ bound on $\sqrt{\varepsilon} \nabla u^{\varepsilon}$ contained in assumption (6.1). In our case, the delicate issue is to pass to the limit in the up-to-the-boundary entropy formulation of (1.3). Our goal is to deduce the characterization (3.5) of entropy solution.

To this end, we reproduce the arguments of [15], but we now take $\xi \in \mathcal{D}([0, T) \times \bar{\Omega}), \xi \geq 0$. We multiply (1.3) by the test function $H_{\alpha}\left(u^{\varepsilon}-k\right) \xi$, where $H_{\alpha}$ is a Lipschitz regularization of $\operatorname{sign}^{+}$(the case of $\operatorname{sign}^{-}$is similar) such that $H_{\alpha}^{\prime}(r)=\frac{1}{\alpha} 1_{(0, \alpha)}(r)$. In addition, we substitute the term $\varphi\left(u^{\varepsilon}\right)$ in $(1.3)$ by $\varphi\left(u^{\varepsilon}\right)-\varphi(k)$, which results in the "new" boundary condition

$$
\left(\varphi\left(u^{\varepsilon}\right)-\varphi(k)-\varepsilon \nabla u^{\varepsilon}\right) \cdot \nu(x) \in \beta_{(t, x)}\left(u^{\varepsilon}\right)-\varphi_{\nu(x)}(k) .
$$

Using the chain rule in time (see, e.g., [1, 28]), using in addition [15, Lemma 1] to make disappear the term $\lim _{\alpha \rightarrow 0^{+}} \int_{\Omega}(\varphi(u)-\varphi(k)) H_{\alpha}^{\prime}\left(u^{\varepsilon}-k\right) \xi$, dropping the positive term $\varepsilon H_{\alpha}^{\prime}\left(u^{\varepsilon}-k\right)\left|\nabla u^{\varepsilon}\right|^{2}$, at the limit $\alpha \rightarrow 0^{+}$we derive the "parabolic up-to-the-boundary entropy equality"

$$
\begin{gathered}
\int_{0}^{T} \int_{\Omega}\left(-\left(u^{\varepsilon}-k\right)^{+} \xi_{t}-q^{+}\left(u^{\varepsilon}, k\right) \cdot \nabla \xi\right)-\int_{\Omega}\left(u_{0}-k\right)^{+} \xi(0, \cdot) \\
\leq-\int_{\Sigma} \operatorname{sign}^{+}\left(u^{\varepsilon}-k\right)\left(b^{\varepsilon}(t, x)-\varphi_{\nu(x)}(k)\right) \xi-\varepsilon \int_{0}^{T} \int_{\Omega} \operatorname{sign}^{+}\left(u^{\varepsilon}-k\right) \nabla u^{\varepsilon} \cdot \nabla \xi
\end{gathered}
$$

with some $b^{\varepsilon}(t, x) \in \beta_{(t, x)}\left(u^{\varepsilon}\right)$ satisfying (6.3). Recall that we have assumed that $f=0$, the general case being similar. In the right-hand side of (6.4), by the monotonicity of $\beta_{(t, x)}$ we have, pointwise on $\Sigma$, the multi-valued inequality

$$
-\operatorname{sign}^{+}\left(u^{\varepsilon}-k\right)\left(b^{\varepsilon}(t, x)-\varphi_{\nu(x)}(k)\right) \leq\left(\beta_{(t, x)}(k)-\varphi_{\nu(x)}(k)\right)^{-} .
$$

Here, the quantity in the right-hand side can be infinite, which makes problematic the localization arguments. Under assumption (6.3) (see Remarks 6.6-6.9 for comments and generalizations) the left-hand side of (6.5) is upper bounded by the $L_{l o c}^{1}([0, T] \times \partial \Omega)$ function defined by $G_{k}(t, x):=$ $G(t, x)+|\varphi(k)|$. Letting $\varepsilon \rightarrow 0^{+}$, from the $L_{l o c}^{1}$ convergence assumption (6.2) we deduce

$$
\begin{aligned}
& \int_{0}^{T} \int_{\Omega}\left(-(u-k)^{+} \xi_{t}-q^{+}(u,\right.k) \cdot \nabla \xi)-\int_{\Omega}\left(u_{0}-k\right)^{+} \xi(0, \cdot) \\
& \leq \int_{0}^{T} \int_{\partial \Omega} G_{k}(t, x) \wedge\left(\beta_{(t, x)}(k)-\varphi_{\nu(x)}(k)\right)^{-} \xi .
\end{aligned}
$$

Now, since $u$ is an entropy solution inside the domain, we can use the strong normal boundary trace $\gamma q_{\nu(x)}^{+}(u, k)$ of $q^{+}(u, k)$ and generate it with the help of the sequence $\left(\xi_{n}\right)_{n}$ of Lemma 3.7. 
The positive test function $\xi$ being arbitrary, we deduce

$$
\gamma q_{\nu(x)}^{+}(u, k) \leq G_{k}(t, x) \wedge\left(\beta_{(t, x)}(k)-\varphi_{\nu(x)}(k)\right)^{-} \mathcal{H}^{N} \text {-a.e. on } \Sigma
$$

(the inequality holds at the Lebesgue points of the left- and right-hand sides). Now notice that we can provide a more precise upper bound for the left-hand side: taking

$$
C_{k}:=2 \max \left\{|\varphi(z)||| z \mid \leq k+\|u\|_{\infty}\right\} \geq\left\|q^{+}(u, k)\right\|_{\infty},
$$

we have $\left|q_{\nu(x)}^{+}(u, k)\right| \leq C_{k}$ pointwise, so that

$$
\gamma q_{\nu(x)}^{+}(u, k) \leq C_{k} \wedge\left(\beta_{(t, x)}(k)-\varphi_{\nu(x)}(k)\right)^{-} .
$$

Combining the entropy inequalities inside the domain (namely, (6.6) with $\xi$ replaced by $\xi\left(1-\xi_{n}\right.$ ), with boundary cut-off functions $\left(\xi_{n}\right)_{n}$ constructed in Lemma 3.7) with (3.11) and (6.7), we finally deduce (3.5).

The simplest example combining Proposition 9.6 and Theorem 6.2 is the following:

Example 6.4. Assume that $\varphi$ satisfies (4.1) and (5.4). Assume that $\beta$ fulfills the analogues of assumptions (5.2) or (5.1) with $\widetilde{D}_{(t, x)}^{ \pm}$replaced by $D_{(t, x)}^{ \pm}$(this makes the assumptions stronger, see Remark 5.1). Assume that the graphs $\beta_{(t, x)}$ are single-valued uniformly bounded on $\mathbb{R}$ functions.

Then for all viscosity parameter $\varepsilon>0$ solutions $u^{\varepsilon}$ of the parabolic problem (1.3) exist; moreover, $u^{\varepsilon}$ converge, as $\varepsilon \rightarrow 0$, to the unique entropy solution of $\left(H_{\varphi, \beta}\left(u_{0}, f\right)\right)$.

The justification of this example is contained in the proof of Theorem 5.2.

Several comments are of order: indeed, we need to discuss generalizations and further applications of Theorem 6.2. First, consider the existence and compactification assumptions (6.1),(6.2).

Remark 6.5.

(i) As we show in Appendix, the existence assumption (6.1) is verified e.g. in the case where $\beta_{(t, x)}$ are monotone continuous functions having $\mathbb{R}$ for their domain. But this assumption is not a necessary one. E.g., existence for the Dirichlet problem for (1.3) is well known, for regular enough bounded Dirichlet data $u^{D}$. If the lack of regularity (in $\left.(t, x)\right)$ of the family $\left(\beta_{(t, x)}\right)_{(t, x) \in \Sigma}$ does not allow for existence of a solution $u^{\varepsilon}$, replacing $\beta$ with a regularized graph $\beta^{\varepsilon}$ (e.g., the Yosida regularization can be used, pointwise in $(t, x))$ one can easily generalize the convergence result of Theorem 6.2 .

(ii) Property (6.2) is ensured in the case where, firstly, the flux $\varphi$ is genuinely non-linear in the sense (5.4); and secondly, a uniform $L^{\infty}$ estimate on the family $\left(u^{\varepsilon}\right)_{\varepsilon}$ is available.

(iii) According to Proposition 9.6(ii) (see also Remark 9.7), uniform $L^{\infty}$ estimates on $u^{\varepsilon}$ are available in the case (9.17) of (9.18) hold. These assumptions exclude important cases. Indeed, for (e.g., homogeneous) Neumann and Robin boundary conditions it is easy to get existence of $u^{\varepsilon}$; but uniform $L^{\infty}$ bounds may require additional restrictions on $\varphi$ : see e.g. the work Bürger, Frid and Karlsen [12] on the Neumann BC case.

(iv) Without $L^{\infty}$ estimates, the issue of convergence of vanishing viscosity approximations becomes quite delicate. E.g., in the case of homogeneous Neumann boundary conditions the family $\left(u^{\varepsilon}\right)_{\varepsilon}$ may be unbounded, and nevertheless converge pointwise to a limit $u \in L^{\infty}\left(Q_{T}\right)$.

In the present paper, we limit our investigation to the case where uniform $L^{\infty}$ bounds (coming from constant sub- and super-solutions) are available. We leave the study of the more delicate situations to a future work.

Further, assumption (6.3) is made in order to simplify the proof of Theorem 6.2 and also because it is enough for the existence result of Theorem 5.2. Assumption (6.3) is of technical nature; unfortunately, it cannot be completely bypassed. We make several comments on (6.3). 
Remark 6.6. Assumption (6.3) is trivially satisfied whenever the graphs $\beta_{(t, x)}$ are uniformly bounded; it also holds if $u^{\varepsilon}$ are uniformly bounded and for all $M>0$, the sets $\beta_{(t, x)}([-M, M])$ are bounded uniformly in $(t, x) \in \Sigma$. A different situation where (6.3) holds is when the sequence $\left(b^{\varepsilon}\right)_{\varepsilon}$ is convergent in $L^{1}(\Sigma)$ (or even in $L_{l o c}^{1}([0, T] \times \partial \Omega)$ ). This was actually the case under the restrictions imposed in our previous works (see [36, 6]).

Remark 6.7. Assumption (6.3) can be replaced by the equi-integrability assumption on $\left(b^{\varepsilon}\right)_{\varepsilon}$. Indeed, setting $G_{k}^{\varepsilon}(t, x):=\left|b^{\varepsilon}(t, x)\right|+|\varphi(k)|$, we get (6.6) with $G_{k}^{\varepsilon}$ in the place of $G_{k}$. The equiintegrability assumption implies that the family of functions $\left(G_{k}^{\varepsilon}(t, x) \wedge\left(\beta_{(t, x)}(k)-\varphi_{\nu(x)}(k)\right)^{-}\right)_{\varepsilon}$ weakly converges to an $L_{l o c}^{1}([0, T] \times \partial \Omega)$ function that we denote $B_{k}$. The proof of Theorem 6.2 leads to the inequalities

$$
\int_{0}^{T} \int_{\partial \Omega} \xi \gamma q_{\nu(x)}^{+}(u, k) \leq \int_{0}^{T} \int_{\partial \Omega} \xi B_{k}
$$

for non-negative continuous compactly supported functions $\xi$ on $\Sigma$. Moreover, $\gamma q_{\nu(x)}^{+}(u, k) \leq C_{k}$, therefore $\gamma q_{\nu(x)}^{+}(u, k) \leq C_{k} \wedge B_{k} \leq C_{k} \wedge\left(\beta_{(t, x)}(k)-\varphi_{\nu(x)}(k)\right)^{-}$pointwise on $\Sigma$.

Remark 6.8. In view of practical application of Theorem 6.2 (which is a conditional result) it would be much useful to replace the domination assumption $(6.3)$ on $\left(b^{\varepsilon}\right)_{\varepsilon}$ by the mere $L_{l o c}^{1}$ boundedness assumption

$$
\int_{0}^{T} \int_{\partial \Omega \cap K}\left|b^{\varepsilon}(t, x)\right| \leq \text { const }_{K} \text { uniformly in } \varepsilon \in(0,1) \text {, for all compact } K \subset \partial \Omega .
$$

E.g. for the case where $\varphi(0)=0, \beta_{(t, x)}(0) \ni 0$ and 0 is in the interior of $\operatorname{Dom} \beta_{(t, x)}$, the bound (6.9) is satisfied automatically. Indeed, under these restrictions, along with the existence result for $u^{\varepsilon}$ (see Proposition 9.6 and Remark 9.7 in Appendix) there comes a uniform estimate

$$
\int_{0}^{T} \int_{\partial \Omega \cap K} b^{\varepsilon}(t, x) u^{\varepsilon}(t, x) \leq \text { const }_{K} .
$$

Due to the monotonicity of $\beta_{(t, x)}$, (6.9) follows readily, while (6.3) is not guaranteed.

With (6.9) in hand the approach of the preceding remark can be applied, but with a locally finite measure replacing the $L_{l o c}^{1}$ function $B_{k}$. Unfortunately, starting from (6.8) with $B_{k}$ a measure the localization argument cannot be continued (see [8, Example 2]).

Remark 6.9. Yet in many important cases, (6.3) is not needed; it can be bypassed whenever the set of finite values of $\beta_{(t, x)}(k)$ is regular enough.

Indeed, introduce $S_{k}^{ \pm}:=\left\{(t, x) \in \Sigma \mid \sup \left( \pm \beta_{(t, x)}\right)<+\infty\right\}$. For instance, assume that for a dense set of values of $k$,

$$
\begin{aligned}
& S_{k}^{ \pm}=E_{k, \infty}^{ \pm} \cup\left(\cup_{M \in \mathbb{N}} E_{k, M}^{ \pm}\right) \text {where } \mathcal{H}^{N}\left(E_{k, \infty}^{ \pm}\right)=0, \\
& \text { the sets } E_{k, M}^{ \pm} \text {are open in } \Sigma \text { and }\left(\beta_{(t, x)}(k)-\varphi_{\nu(x)}(k)\right)^{ \pm} \leq M \text { on } E_{k, M}^{ \pm} .
\end{aligned}
$$

Under this assumption, we can simply localize inequalities (6.4) using test functions $\xi$ such that $\left.\xi\right|_{\Sigma}$ is supported in $E_{k, M}^{-}$and then apply (6.5) in the situation where its right-hand side is finite. Then we directly get

$$
\begin{aligned}
& \gamma q_{\nu(x)}^{+}(u, k) \leq\left(\beta_{(t, x)}(k)-\varphi_{\nu(x)}(k)\right)^{-} \\
& \mathcal{H}^{N} \text {-a.e. on } S_{k}^{-} \equiv\left\{(t, x) \mid\left(\beta_{(t, x)}(k)-\varphi_{\nu(x)}(k)\right)^{-} \neq+\infty\right\}
\end{aligned}
$$

being understood that we have $q_{\nu(x)}^{+}(u, k) \leq C_{k}$ on the complementary of this set. This establishes (6.7) and complements the proof of Theorem 6.2 with assumption (6.3) replaced by (6.10). 
Such modification of Theorem 6.2 can be applied, e.g., to Dirichlet or obstacle problems under mild regularity assumptions on the boundary data. Indeed, the existence result for the Dirichlet problem is well known, as well as the uniform $L^{\infty}$ bound on $u^{\varepsilon}$. Assumption (6.3) of Theorem 6.2 is circumvented in the way of Remark 6.9. To be specific, for the Dirichlet graphs $\beta_{(t, x)}=\left\{u^{D}(t, x)\right\} \times \mathbb{R}$ property (6.10) is fulfilled for continuous and even for piecewise continuous $u^{D}$ (yet it is not fulfilled in the case of "fractal" data $u^{D}$ ).

Example 6.10. (cf. Bardos, LeRoux and Nédélec [9] and Vasseur [39])

Assume that $\Omega$ is bounded, $\varphi$ satisfies (5.4), and $u_{0} \in L^{\infty}(\Omega)$. Assume that $\beta$ is the Dirichlet graph corresponding to a piecewise continuous datum $u^{D} \in L^{2}\left(0, T ; H^{1 / 2}(\partial \Omega)\right) \cap L^{\infty}(\Sigma)$.

Then for all viscosity parameter $\varepsilon>0$ solutions $u^{\varepsilon}$ of the parabolic problem (1.3) exist; then $u^{\varepsilon}$ converge, as $\varepsilon \rightarrow 0$, to the unique entropy solution of $\left(H_{\varphi, \beta}\left(u_{0}, f\right)\right)$.

Analogous results hold for the case of obstacle boundary conditions with piecewise continuous data $u^{m}, u^{M} \in \cap L^{2}\left(0, T ; H^{1 / 2}(\partial \Omega)\right) \cap L^{\infty}(\Sigma)$.

6.2. Stability of the notion of entropy solution. Let us consider a sequence of problems of the kind $\left(H_{\varphi, \beta}\left(u_{0}, f\right)\right)$ associated with data $u_{0}^{\delta}, f^{\delta}$ and nonlinearities $\varphi^{\delta}, \beta^{\delta}$ (here $\delta$ is a parameter; for the sake of being definite assume that $\delta$ is positive and converges to zero). We assume that there exist associated entropy solutions $u^{\delta}$; we want to deduce an entropy formulation for an accumulation point $u$ of $u^{\delta}$, assuming ad hoc convergence of $u_{0}^{\delta}, f^{\delta}, \varphi^{\delta}, \beta^{\delta}$ to some limits $u_{0}^{\delta}, f^{\delta}, \varphi^{\delta}, \beta^{\delta}$.

In the three paragraphs $\S 6.2 .1-6.2 .3$ below, we will demonstrate three different kinds of heredity for the notion of entropy solution: the one coming from compactification of $\left(u^{\delta}\right)_{\delta}$ (due to a genuine nonlinearity assumption on the flux $\varphi$ ); the one coming from monotone approximation procedures; and the one where the $L^{1}$ contraction property of Theorem 4.1 makes $\left(u^{\delta}\right)_{\delta}$ a Cauchy sequence. Because we now treat of a hyperbolic problem, the boundary condition can be encoded either by the formal BC graphs $\beta_{(t, x)}$ (via formulation (3.5)) or by the projected graphs $\tilde{\mathcal{B}}_{(t, x)}=$ $\widetilde{\mathcal{P}}_{x} \beta_{(t, x)}$ that directly describe the effective BC. We will exploit the two possibilities.

In $\S 6.2 .1$, we will need a notion of convergence of maximal monotone graphs. Let us take the following:

$$
\begin{aligned}
& \beta_{(t, x)}^{\delta} \rightarrow \beta_{(t, x)} \text { as } \delta \rightarrow 0 \text { if } \lim _{\delta \rightarrow 0} \inf \beta_{(t, x)}^{\delta}(k) \leq \beta_{(t, x)}(k) \leq \lim _{\delta \rightarrow 0} \sup \beta_{(t, x)}^{\delta}(k) \\
& \text { at every point } k \text { of continuity of } \beta_{(t, x)}(k) .
\end{aligned}
$$

This assumption is satisfied, e.g., if $\beta_{(t, x)}^{\delta}$ are the Yosida approximations of $\beta_{(t, x)}$ (Yosida approximation is a classical way for regularizing maximal monotone graphs; see, e.g., [37] and § 6.2.1). A different notion of convergence of $\beta^{\delta}$ can be given in terms of the distance $d_{x}$ between the projected graphs $\widetilde{\mathcal{P}}_{x} \beta^{\delta}$; it is used in $\S 6.2 .3$, and the corresponding order relation is exploited in $\S 6.2 .2$.

Throughout the section, we assume that

$$
\exists M>0\left\|u^{\delta}\right\|_{\infty} \leq M \text { uniformly in } \delta .
$$

(in particular, $\left\|u_{0}^{\delta}\right\|_{\infty}$ and $\int_{0}^{T}\left\|f^{\delta}(t, \cdot)\right\|_{\infty} d t$ should obey uniform in $\delta$ bounds). In order to enforce the $L^{\infty}$ estimate (6.12), we actually assume that either (5.1) or (5.2) is fulfilled (with $\delta$-dependent $f$ and the sets $\left.\widetilde{D}_{(t, x)}^{ \pm}\right)$.

Lemma 6.11. Assume (4.1).

(i) Assume $\left\|u_{0}^{\delta}\right\|_{\infty}$ and $\int_{0}^{T}\left\|f^{\delta}(t, \cdot)\right\|_{\infty} d t$ are bounded uniformly in $\delta$ and assumption (5.2) holds with $\mathcal{A}^{ \pm}$independent of $\delta$. Then (6.12) holds. 
(ii) Assume that $\left\|u_{0}^{\delta}\right\|_{\infty}$ is bounded uniformly in $\delta$ and assumption (5.1) holds with $\left(A_{m}^{ \pm}\right)_{m}$ independent ${ }^{13}$ of $\delta$. Then (6.12) holds.

The lemma is shown by using the comparison principle of Theorem 4.1 and the appropriate sub- and super-solutions of problems $\left(H_{\varphi^{\delta}, \beta^{\delta}}\left(u_{0}^{\delta}, f^{\delta}\right)\right)$ that stem either from assumption (5.1) (constants $A_{m}^{ \pm}$are used) or from assumption (5.2) (in this case, the construction described in Remark 9.7 is used).

6.2.1. Heredity by compactness. In this paragraph, let us assume the following properties:

$u_{0}^{\delta} \quad$ converge to $u_{0}$ in $L_{l o c}^{1}(\Omega), \quad f^{\delta}$ converge to $f$ in $L_{l o c}^{1}\left(Q_{T}\right)$,

$\varphi^{\delta}$ converge to $\varphi$ uniformly on every compact interval of $\mathbb{R}$, and for $\mathcal{H}^{N}$-a.e. $(t, x) \in \Sigma, \quad \beta_{(t, x)}^{\delta} \rightarrow \beta_{(t, x)}$ in the sense (6.11).

(note that it is enough to assume relative compactness of $\left(u^{\delta}\right)_{\delta}$ and of $\left.\left(f^{\delta}\right)_{\delta}\right)$.

Proposition 6.12. Assume the data $u_{0}^{\delta}, f^{\delta}, \varphi^{\delta}, \beta^{\delta}$ converge in the sense (6.13). Assume (5.3) or (5.1) hold with $A$ or $\left(A_{m}^{ \pm}\right)_{m}$ that are suitable for $\varphi^{\delta}, \beta^{\delta}$ simultaneously for all $\delta>0$. Assume $\varphi$ is genuinely nonlinear in the sense (5.4), and assume that (4.1) holds.

Assume $\left\|u_{0}^{\delta}\right\|_{\infty}$ is uniformly bounded; in the case (5.3) assume $\int_{0}^{T}\left\|f^{\delta}(t, \cdot)\right\|_{\infty} d t$ is uniformly bounded. Consider a family $\left(u^{\delta}\right)_{\delta}$ of entropy solutions of $\left(H_{\varphi^{\delta}, \beta^{\delta}}\left(u_{0}^{\delta}, f^{\delta}\right)\right)$. Then there exists an accumulation point $u$ of $\left(u^{\delta}\right)_{\delta}$, as $\delta \rightarrow 0$, and $u$ is an entropy solution of the limit problem $\left(H_{\varphi, \beta}\left(u_{0}, f\right)\right)$.

Proof. First of all, the uniform $L^{\infty}$ bound (6.12) follows by Lemma 6.11. Then $L_{l o c}^{1}$ relative compactness of $\left(u^{\delta}\right)_{\delta}$ is a consequence of the convergence of $\varphi^{\delta}$ to $\varphi$ and of assumption (5.4) (see, e.g., [29, 32] and [23]). It remains to pass to the limit in the entropy formulation for $H_{\varphi^{\delta}, \beta^{\delta}}\left(u_{0}^{\delta}, f^{\delta}\right)$; to do this, we pick the up-to-the-boundary entropy inequalities (3.5) written for $u^{\delta}$. Let us focus on the case of the entropies $(\cdot-k)^{+}$; the case of $(\cdot-k)^{-}$is analogous. Passage to the limit in the left-hand side is straightforward, using (6.13). Thus we only have to establish that, for $\xi \in \mathcal{D}(\Sigma)$,

$$
\begin{aligned}
\liminf _{\delta \rightarrow 0} \iint_{\Sigma} C_{k} \wedge\left(\inf \beta_{(t, x)}^{\delta}(k)-\varphi_{\nu(x)}^{\delta}(k)\right)^{-} \xi \\
\leq \iint_{\Sigma} C_{k} \wedge\left(\inf \beta_{(t, x)}(k)-\varphi_{\nu(x)}(k)\right)^{-} \xi
\end{aligned}
$$

(see the first point of Remark 3.4). Recall that $C_{k}$ may depend only on $k,\left\|u^{\delta}\right\|_{\infty}$ and a local bound of $\left|\varphi^{\delta}\right|$, thus we can take $C_{k}$ independent of $\delta$. Consequently, the dominated convergence theorem for (6.14) can be used.

According to Lemma 6.13 below, convergence (6.11) of $\beta^{\delta}$ does imply that for a.e. $k \in \mathbb{R}$, there holds $\inf \beta_{(t, x)}^{\delta}(k) \rightarrow \inf \beta_{(t, x)}(k)$ for $\mathcal{H}^{N}$-a.e. $(t, x) \in \Sigma$ as $\delta \rightarrow 0$ (the convergence takes place in $\overline{\mathbb{R}})$. Since $\varphi^{\delta}(k)$ tends to $\varphi(k)$, the left-hand side integrand in (6.14) converges $\mathcal{H}^{N}$-a.e. to the integrand of the right-hand side. This justifies (6.14) for all $k \in \mathbb{R}$ except, may be, for a set of measure zero. Because the left-hand side of (3.5) is continuous in $k$, one readily extends (3.5) to all $k \in \mathbb{R}$. This ends the proof.

Lemma 6.13. Under assumption (1.1), a.e. point $k \in \mathbb{R}$ is a continuity point of $\beta_{(t, x)}$ simultaneously for $\mathcal{H}^{N}$-a.e. $(t, x) \in \Sigma$.

\footnotetext{
${ }^{13}$ This assumption can be generalized; e.g., it is enough that $c_{m} \leq \pm A_{m}^{ \pm, \delta} \leq C_{m}$ with $c_{m} \rightarrow \infty$ as $m \rightarrow \infty$.
} 
Proof. Consider the $[0,+\infty]$-valued map $\theta(t, x ; k):=\sup \beta_{(t, x)}(k)-\inf \beta_{(t, x)}(k)$; it is measurable on $\Sigma \times \mathbb{R}$ due to (1.1). Indeed, by (1.1), for all $\ell, c \in \mathbb{R}, m_{\ell}(c):=\left\{(t, x) \in \Sigma \mid \inf \beta_{(t, x)}(\ell)>c\right\}$ is an $\mathcal{H}^{N}$-measurable subset of $\Sigma$, thus $m_{\ell}(c) \times[\ell,+\infty]$ is measurable on $\Sigma \times \overline{\mathbb{R}}$ wrt to the measure $\mathcal{H}^{N} \otimes \mu$ where $\mu$ is given, for instance, by $\mu([a, b)):=\arctan b-\arctan a$. Now, due to the lower semicontinuity of the map $\ell \mapsto \inf \beta_{(t, x)}(\ell)$, the sets $\left\{k \mid \inf \beta_{(t, x)}(k)>c\right\}$ are open. Therefore

$$
\left\{(t, x ; k) \mid \inf \beta_{(t, x)}(k)>c\right\}=\bigcup_{\ell \in \mathbb{Q}} m_{\ell}(c) \times[\ell,+\infty],
$$

which is a countable union of measurable sets. Hence it is measurable on $\Sigma \times \mathbb{R}$ wrt $\mathcal{H}^{N} \otimes \mu$, thus $(t, x ; k) \mapsto \inf \beta_{(t, x)}(k)$ is measurable.

Now, for all $\sigma=(t, x) \in \Sigma, \theta(t, x ; \cdot)$ is zero $\mu$-a.e on $\mathbb{R}$ due to the monotonicity of $\beta_{(t, x)}$. Applying the Fubini-Tonnelli theorem, we see that $\int_{\mathbb{R}}\left(\int_{\Sigma} \theta(\sigma ; k) d \mathcal{H}^{N}(\sigma)\right) d \mu(k)=0$. Thus for a.e. $k \in \mathbb{R}$, the function $\theta(\cdot ; k)$ is zero $\mathcal{H}^{N}$-a.e. on $\Sigma$, which was to be proved.

Now let us give an application of Proposition 6.12 to a Yosida-like approximation of $\beta$.

Example 6.14. Assume that $\varphi$ satisfies (4.1) and (5.4). Assume that $\beta$ fulfills the analogues of assumptions (5.2) or (5.1) with $\widetilde{D}_{(t, x)}^{ \pm}$replaced by $D_{(t, x)}^{ \pm}$(this makes the assumptions stronger). Then entropy solution $u$ of $\left(H_{\varphi, \beta}\left(u_{0}, f\right)\right)$ is the limit of $u^{\delta}$, where $u^{\delta}$ are limits of the vanishing viscosity method applied to problems $\left(H_{\varphi, \beta^{\delta}}\left(u_{0}, f\right)\right)$ with $\beta^{\delta}$ the approximation $(6.15)$ of $\beta$ that we describe below.

Indeed, consider, e.g., the case where (5.1) holds with $D_{(t, x)}^{ \pm}$in the place of $\widetilde{D}_{(t, x)}^{ \pm}$, and pick $m$ such that $u_{0}$ takes values in $\left[A_{m}^{-}, A_{m}^{+}\right]$. Then there exist $b_{m}^{ \pm}(t, x) \in \beta_{(t, x)}\left(A_{m}^{ \pm}\right)$such that $b_{m}^{+}(t, x) \geq \varphi_{\nu(x)}\left(A_{m}^{+}\right)$and $b_{m}^{-}(t, x) \leq \varphi_{\nu(x)}\left(A_{m}^{-}\right)$Without loss of generality, we may assume that $\pm b_{m}^{+}(t, x)>0$ (otherwise we can modify $\beta$ without changing the effective $\mathrm{BC}$ graph $\tilde{\beta}$ in the interval $\left[A_{m}^{-}, A_{m}^{+}\right]$, as in the case of truncations (5.5)). We can use the result of Theorem 6.2 for the case of single-valued continuous graphs $\beta_{m}^{\delta}, \delta>0$, defined as follows:

$$
\beta_{m}^{\delta}=\left\{(z, b) \mid z+\delta b_{m}^{+} \frac{z^{+}}{A_{m}^{+}}-\delta b_{m}^{-} \frac{z^{-}}{A_{m}^{-}} \in \beta^{-1}(b)+\delta b\right\} ;
$$

(here we have skipped the parameters $(t, x) \in \Sigma$ ). Approximation (6.15) ensures the convergence property (6.11). It is inspired by the Yosida approximation $\beta^{\delta}=\left(\beta^{-1}+\delta I\right)^{-1}$, but by construction, it has the additional property that $\beta_{m}^{\delta}\left(A_{m}^{ \pm}\right)=b_{m}^{ \pm}$. Therefore $A_{m}^{ \pm} \in D_{(t, x)}^{ \pm, \delta}$ for all $(t, x) \in \Sigma$; hence we can use the truncation convention (5.5) simultaneously for all $\delta$. Applying Proposition 6.12, we deduce that solutions $u^{\delta}$ of $\left(H_{\varphi, \beta^{\delta}}\left(u_{0}, f\right)\right)$ ( $u^{\delta}$ being obtained via Theorem 6.2) converge to the unique entropy solution of $\left(H_{\varphi, \beta}\left(u_{0}, f\right)\right)$.

6.2.2. Heredity by monotonicity. In this paragraph, let us assume the following properties:

$$
\begin{aligned}
& \varphi^{\delta}=\varphi \text { for all } \delta, \quad u_{0}^{\delta} \downarrow_{\delta \rightarrow 0} u_{0}, \quad f^{\delta} \downarrow_{\delta \rightarrow 0} f, \\
& \text { and for } \mathcal{H}^{N} \text {-a.e. }(t, x) \in \Sigma, \quad \beta_{(t, x)}^{\delta} \succeq_{x} \beta_{(t, x)}^{\alpha} \text { if } 0<\delta \leq \alpha .
\end{aligned}
$$

The case where $u_{0}^{\delta} \uparrow_{\delta \rightarrow 0} u_{0}, f^{\delta} \uparrow_{\delta \rightarrow 0} f$ and $\beta_{(t, x)}^{\delta} \downarrow_{\delta \rightarrow 0}$ (in the sense $\succeq_{x}$ ) can be considered in the same way. In this paragraph, we will work with projected graphs $\widetilde{\mathcal{B}}_{(t, x)}^{\delta}$ in the place of $\beta_{(t, x)}^{\delta}$.

By Definition 2.7, we have $\widetilde{\mathcal{B}}_{(t, x)}^{\delta} \geq \widetilde{\mathcal{B}}_{(t, x)}^{\alpha}$ pointwise on $\mathbb{R}$, if $0<\delta<\alpha$. Therefore it is automatic that, for a.e. $(t, x), \widetilde{\mathcal{B}}_{(t, x)}^{\delta} \uparrow \Psi_{(t, x)}$ as $\delta \rightarrow 0$, with some non-decreasing function $\Psi_{(t, x)}$ which could possibly be infinite. Under the assumptions we take, we can truncate $\varphi$, $\beta$ (which means that, e.g., (5.5) is assumed) without changing the solutions $u^{\delta}$. It follows that $\Psi_{(t, x)}$ is finite because bounded by $\|\varphi\|_{\infty}<\infty$. Finally, recall that for all $\delta>0, \widetilde{\mathcal{B}}_{(t, x)}^{\delta}$ 
is a continuous monotone function that is constant on every connected component of the set $\left\{k \in \mathbb{R} \mid \widetilde{\mathcal{B}}_{(t, x)}^{\delta}(k) \neq \varphi_{\nu(x)}(k)\right\}$. It is easy to see that this structure is inherited by passage to a monotone limit, therefore in the sequel we will write $\widetilde{\mathcal{B}}_{(t, x)}$ in the place of $\Psi$.

The compactness of $\left(u^{\delta}\right)_{\delta}$ is automatic from its monotonicity, ensured by the comparison principle of Theorem 4.1. We have

Proposition 6.15. Assume the data $u_{0}^{\delta}, f^{\delta}, \beta^{\delta}$ converge monotonically, in the sense (6.16). Assume (5.1) or (5.2) hold, with $\left(A_{m}^{ \pm}\right)_{m}$ or $\mathcal{A}^{ \pm}$that are suitable for $\varphi^{\delta}, \beta^{\delta}$ simultaneously for all $\delta>0$. Assume that (4.1) holds.

Assume $\left\|u_{0}^{\delta}\right\|_{\infty}$ is uniformly bounded; in the case (5.2) assume $\int_{0}^{T}\left\|f^{\delta}(t, \cdot)\right\|_{\infty} d t$ is uniformly bounded. Consider a family $\left(u^{\delta}\right)_{\delta}$ of entropy solutions of $\left(H_{\varphi, \beta^{\delta}}\left(u_{0}^{\delta}, f^{\delta}\right)\right)$. Then there exists a limit $u$ of $u^{\delta}$, as $\delta \rightarrow 0$, and $u$ is an entropy solution of the limit problem $\left(H_{\varphi, \beta}\left(u_{0}, f\right)\right)$ with the graph $\beta$ given by $\beta_{(t, x)}:=\widetilde{\mathcal{B}}_{(t, x)}=\lim _{\delta \rightarrow 0} \widetilde{\mathcal{B}}_{(t, x)}^{\delta}$.

Proof. The uniform $L^{\infty}$ bound (6.12) follows by Lemma 6.11. By Theorem 4.1 and due to the monotone convergence assumption (6.16) on the data, we deduce that $u^{\delta} \leq u^{\alpha}$ a.e. on $Q_{T}$ for $0<\delta \leq \alpha$. Thus $u:=\lim _{\delta \rightarrow 0} u^{\delta}$ is well defined a.e. on $Q_{T}$ (one can start by considering a sequence $\left(\delta_{n}\right)_{n}$ decreasing to zero; at the very end, we find that $u$ is an entropy solution of $\left(H_{\varphi, \beta}\left(u_{0}, f\right)\right)$, which ensures the uniqueness of an accumulation point of $\left.\left(u^{\delta}\right)_{\delta}\right)$.

As in Proposition 6.12, we readily pass to the limit in the left-hand side of the entropy formulation (3.5) written for $u^{\delta}$. In the right-hand side, we can choose to write

$$
\left(\widetilde{\mathcal{B}}_{(t, x)}^{\delta}(k)-\varphi_{\nu(x)}(k)\right)^{\mp} \text { in the place of }\left(\beta_{(t, x)}^{\delta}(k)-\varphi_{\nu(x)}(k)\right)^{\mp} .
$$

Indeed, $u^{\delta}$, being the entropy solution corresponding to $\beta_{(t, x)}$, is also the entropy solution corresponding to the graph $\widetilde{\mathcal{B}}_{(t, x)}^{\delta}$ (both graphs lead to the same admissible traces set $\widetilde{D}_{(t, x)}^{0}$ ). Then by the monotone convergence theorem we readily pass to the limit in the right-hand side of inequalities (3.5) written for $u^{\delta}$. The proof is complete.

Remark 6.16. It is easy to check that for all $x \in \partial \Omega$ the projection $\widetilde{\mathcal{P}}_{x}$ on $\mathbb{B}$ is an orderpreserving operator. Therefore the monotonicity property of $\beta_{(t, x)}^{\delta}$ in the sense of the relation $\succeq_{x}$ is implied by its monotonicity in $\delta$ in the pointwise (multi-valued) sense. In this case the limit $u$ of Proposition 6.15 is also an entropy solution of $\left(H_{\varphi, \beta}\left(u_{0}, f\right)\right)$ with the graph $\beta_{(t, x)}$ obtained as $\lim _{\delta \rightarrow 0} \beta_{(t, x)}^{\delta}$ (the limit here is in the sense $(6.11)$ ).

The following example complements Example 6.14. The corresponding existence claim eventually attains the same generality that the result of Theorem 5.2.

Example 6.17. Assume that $\varphi$ satisfies (4.1) and (5.4). Assume that $\beta$ fulfills (5.2) or (5.1).

There exists a family of bi-monotone graphs $\left(\beta^{\delta, \lambda}\right)_{\delta, \lambda>0}$ which satisfies the assumptions of Ex-

ample 6.14. The entropy solutions $u^{\delta, \lambda}$ (that can be constructed, e.g., in the way of Example 6.14) of the associated problem $\left(H_{\varphi, \beta^{\delta, \lambda}}\left(u_{0}, f\right)\right)$ converge to an entropy solution $u$ of $\left(H_{\varphi, \beta}\left(u_{0}, f\right)\right)$ as $\delta, \lambda$ tend to zero.

Indeed, assume, e.g., that $\beta$ satisfies (5.1). We approximate $\beta$ by $\beta^{\delta, \lambda}:=\beta+\partial I_{[-1 / \delta, 1 / \lambda]}$, where $\partial I_{[a, b]}$ (the subdifferential of the indicator function of $[a, b]$ ) is the obstacle graph corresponding to the interval $[a, b]$. This ensures that

$$
\beta^{\delta, \lambda}(k)=+\infty \geq \varphi_{\nu(x)}(k) \text { for } k>1 / \lambda \text { and } \beta^{\delta, \lambda}(k)=-\infty \leq \varphi_{\nu(x)}(k) \text { for } k<-1 / \delta,
$$

so that $(-\infty,-1 / \delta] \subset D_{(t, x)}^{-, \delta, \lambda}$ and $[1 / \lambda,+\infty) \subset D_{(t, x)}^{+, \delta, \lambda}$ for all $(t, x) \in \Sigma$. Thus $\beta^{\delta, \lambda}$ fulfills the assumptions of Example 6.14. 
Furthermore, whenever

$$
-1 / \delta \in D_{(t, x)}^{-} \text {and } 1 / \lambda \in D_{(t, x)}^{+},
$$

as in the localization procedure in the proof of Theorem 5.2 we see that $\widetilde{\mathcal{B}}_{(t, x)}^{\delta, \lambda}:=\widetilde{\mathcal{P}}_{x} \beta_{(t, x)}^{\delta, \lambda}$ coincides with $\widetilde{\mathcal{B}}_{(t, x)}$ in the interval $[-1 / \delta, 1 / \lambda]$. Due to assumption (5.1), we can construct sequences of parameters $\delta$ and $\lambda$ going to zero and satisfying (6.17). Moreover, due to (5.1) solutions $u^{\delta, \lambda}$ constructed in Example 6.14 take their values within some fixed interval $[-M, M]$.

By construction, $\left(\beta_{(t, x)}^{\delta, \lambda}\right)_{\delta>0}$ decreases as $\delta \downarrow 0$ for every $\lambda>0$; and $\left(\beta_{(t, x)}^{\delta, \lambda}\right)_{\lambda>0}$ increases as $\lambda \downarrow 0$ for every $\delta>0$. As $\delta \rightarrow 0$, we can use Proposition 6.15 to infer that $u^{\delta, \lambda} \uparrow_{\delta \rightarrow 0^{+}} u^{0, \lambda}$ and $u^{0, \lambda}$ is the entropy solution associated with the graph $\beta^{0, \lambda}:=\beta+\partial I_{(-\infty, 1 / \lambda]}$. As $\lambda \rightarrow 0$, using the analogue of Proposition 6.15 we deduce that $u^{0, \lambda} \downarrow_{\lambda \rightarrow 0^{+}} u$ and $u$ is the unique entropy solution of problem $\left(H_{\varphi, \beta}\left(u_{0}, f\right)\right)$. Exchanging the order of passage to the limit, we also get $u^{\delta, \lambda} \downarrow_{\lambda \rightarrow 0^{+}} u^{\delta, 0} \uparrow_{\delta \rightarrow 0^{+}} u$. By the squeeze lemma, we infer that $u^{\delta, \lambda} \rightarrow u$ as $(\delta, \lambda) \rightarrow(0,0)$.

6.2.3. Heredity by $L^{1}$ contraction. In this paragraph, let us assume the following properties:

$$
\begin{aligned}
& \varphi^{\delta}=\varphi \text { for all } \delta, \quad u_{0}^{\delta}-u_{0} \rightarrow 0 \text { in } L^{1}(\Omega), \quad f^{\delta}-f \rightarrow 0 \text { in } L^{1}\left(Q_{T}\right), \\
& \text { and for } \mathcal{H}^{N} \text {-a.e. }(t, x) \in \Sigma, \quad d_{x}\left(\beta_{(t, x)}^{\delta}, \beta_{(t, x)}\right) \rightarrow 0 \text { as } \delta \rightarrow 0
\end{aligned}
$$

with $d_{x}\left(\beta_{(t, x)}^{\delta}, \beta_{(t, x)}\right) \equiv d_{x}\left(\tilde{\beta}_{(t, x)}^{\delta}, \tilde{\beta}_{(t, x)}\right)$ given by Definition 2.7. The practical interpretation of the above convergence is therefore,

$$
\widetilde{\mathcal{B}}_{(t, x)}^{\delta}=\widetilde{\mathcal{P}}_{x} \beta_{(t, x)}^{\delta} \longrightarrow \widetilde{\mathcal{B}}_{(t, x)}=\widetilde{\mathcal{P}}_{x} \beta_{(t, x)} \text { uniformly on } \mathbb{R} .
$$

The convergence of $\left(u^{\delta}\right)_{\delta}$ follows by the $L^{1}$ contraction principle of Theorem 4.1. We have

Proposition 6.18. Assume the data $u_{0}^{\delta}, f^{\delta}, \beta^{\delta}$ converge in the sense (6.18). Assume that (5.1) or (5.2) hold, with $\left(A_{m}^{ \pm}\right)_{m}$ or $\mathcal{A}^{ \pm}$that are suitable for $\varphi^{\delta}, \beta^{\delta}$ simultaneously for all $\delta>0$. Assume that (4.1) holds.

Assume $\left\|u_{0}^{\delta}\right\|_{\infty}$ is uniformly bounded; in the case (5.2) assume $\int_{0}^{T}\|f(t, \cdot)\|_{\infty} d t$ is uniformly bounded. Consider a family $\left(u^{\delta}\right)_{\delta}$ of entropy solutions of $\left(H_{\varphi, \beta^{\delta}}\left(u_{0}^{\delta}, f^{\delta}\right)\right)$. Then there exists a limit $u$ of $u^{\delta}$, as $\delta \rightarrow 0$, and $u$ is an entropy solution of the limit problem $\left(H_{\varphi, \beta}\left(u_{0}, f\right)\right)$.

Proof. As in Propositions 6.12,6.15, the $L^{\infty}$ bound (6.12) is immediate. To continue, from inequalities (4.2) of Theorem 4.1 we deduce

$$
\int_{\Omega}\left|u^{\delta}-u^{\alpha}\right|(t) \leq \int_{\Omega}\left|u_{0}^{\delta}-u_{0}^{\alpha}\right|+\int_{0}^{t} \int_{\Omega}\left|f^{\delta}-f^{\alpha}\right|+\int_{0}^{t} \int_{\partial \Omega} d_{x}\left(\beta_{(t, x)}^{\delta}, \beta_{(t, x)}^{\alpha}\right) .
$$

By the triangular inequality (recall that $d_{x}$, when used on $\overline{\mathbb{B}}_{x}$, is a distance) and the convergence properties (6.18) we see that the right-hand side of (6.20) tends to zero as $\max \{\delta, \alpha\} \rightarrow 0$. Thus by the Cauchy criterion, $\left(u_{\delta}\right)_{\delta}$ converges in $L^{\infty}\left(0, T ; L^{1}(\Omega)\right)$, as $\delta \rightarrow 0$, to some limit $u$. Then $u$ fulfills (3.5). Indeed, the passage to the limit in up-to-the-boundary entropy inequalities (3.5) written for $u^{\delta}$ is straightforward. In particular, in the right-hand side we can substitute $\beta^{\delta}(k)$ by $\widetilde{\mathcal{B}}^{\delta}(k)$; the latter expression converges to $\widetilde{\mathcal{B}}(k)$, due to $(6.19)$. We conclude using the dominated convergence theorem.

A trivial application of $(6.18)$ is, approximate the initial data $u_{0}$ and source data $f$. Let us give another application which is suitable, e.g., for approximation in the sense of the Lusin theorem of merely measurable Dirichlet or obstacle boundary data by continuous in $(t, x)$ data: 
Example 6.19. Assume that (5.1) or (5.2) hold, with $\left(A_{m}^{ \pm}\right)_{m}$ or $\mathcal{A}^{ \pm}$that are suitable for $\varphi^{\delta}, \beta^{\delta}$ simultaneously for all $\delta>0$. Assume that (4.1) holds.

Assume that $\beta^{\delta} \rightarrow \beta$ in the following sense:

$$
\text { the } \mathcal{H}^{N} \text { measure of the set } R_{\delta}:=\left\{(t, x) \in \Sigma \mid \beta_{(t, x)}^{\delta} \neq \beta_{(t, x)}\right\} \text { vanishes as } \delta \rightarrow 0 \text {. }
$$

Then solution $u^{\delta}$ of problem $\left(H_{\varphi, \beta^{\delta}}\left(u_{0}, f\right)\right)$ tends, as $\delta \rightarrow 0$, to a limit $u$ that solves problem $\left(H_{\varphi, \beta}\left(u_{0}, f\right)\right)$.

This result follows readily from Proposition 6.18: indeed, $u_{0}, f$ being fixed, (6.21) gives (6.18).

The next application, that complements Example 6.10, uses pointwise approximation of the obstacle problem (the case $u^{m}=u^{M}$ of the obstacle problem yields the Dirichlet problem).

Example 6.20. In the setting of Example 6.19, in the place of (6.21) assume that $\beta^{\delta}$ is the obstacle graph

$$
\beta_{(t, x)}^{\delta}=\left(\left\{u_{\delta}^{m}(t, x)\right\} \times \mathbb{R}^{-}\right) \cup\left(\left[u_{\delta}^{m}(t, x), u_{\delta}^{M}(t, x)\right] \times\{0\}\right) \cup\left(\left\{u_{\delta}^{M}(t, x)\right\} \times \mathbb{R}^{+}\right) .
$$

Assume that $u_{\delta}^{m}$ and $u_{\delta}^{M}$ obey uniform $L^{\infty}$ bounds and converge $\mathcal{H}^{N}$-a.e. on $\Sigma$ to limits $u^{m}$ and $u^{M}$, respectively. Then solutions $u^{\delta}$ of problem $\left(H_{\varphi, \beta^{\delta}}\left(u_{0}, f\right)\right)$ converge to a limit $u$ that solves problem $\left(H_{\varphi, \beta}\left(u_{0}, f\right)\right)$ with the obstacle graph $\beta$ corresponding to $u^{m}, u^{M}$.

The proof is straightforward, taking into account the following lemma:

Lemma 6.21. Assume $\beta^{\delta}, \beta$ are obstacle graphs corresponding to $u_{\delta}^{m}, u^{m}$ and $u_{\delta}^{M}, u^{M}$ that take values in some compact subset I of $\mathbb{R}$. Let $\omega_{\varphi}: \mathbb{R}^{+} \mapsto \mathbb{R}^{+}$is the modulus of continuity of $\varphi$ on I. Then for all $(t, x) \in \Sigma$ there holds $d_{x}\left(\beta^{\delta}, \beta\right) \leq \omega_{\varphi}\left(\max \left\{\left|u_{\delta}^{m}-u^{m}\right|,\left|u_{\delta}^{M}-u^{M}\right|\right\}\right)$.

The proof relies on the fact that $\widetilde{\mathcal{B}}_{(t, x)}^{\delta}(z)$ and $\widetilde{\mathcal{B}}_{(t, x)}(z)$ coincide except when their values fall within one of the two strips

$$
S^{m}:=\varphi_{\nu(x)}\left(\left[\min \left\{u^{m}, u_{\delta}^{m}\right\}, \max \left\{u^{m}, u_{\delta}^{m}\right\}\right) \text { and } S^{M}:=\varphi_{\nu(x)}\left(\left[\min \left\{u^{M}, u_{\delta}^{M}\right\}, \max \left\{u^{M}, u_{\delta}^{M}\right\}\right]\right) ;\right.
$$

then $d_{x}\left(\beta^{\delta}, \beta\right)=\left\|\widetilde{\mathcal{B}}_{(t, x)}^{\delta}-\widetilde{\mathcal{B}}_{(t, x)}\right\|_{\infty}$ is less than or equal to the width of the strips, which does not exceed $\omega_{\varphi}\left(\max \left\{\left|u_{\delta}^{m}-u^{m}\right|,\left|u_{\delta}^{M}-u^{M}\right|\right\}\right)$.

\section{FURTher EXISTENCE AND CONVERGENCE RESUlts}

Here we explore convergence of approximations in two complementary directions. In Section 7.1 we discard the genuine nonlinearity assumption (5.4) and exploit $B V_{l o c}$ estimates for proving compactness. This technique is limited to one-dimensional case (with a simple generalization to half-space or strip domains). In Section 7.2 we set up a framework for studying measure-valued (entropy-process) solutions, so that to replace the strong compactness in $L_{l o c}^{1}$ of sequences of approximate solutions by their weak-* compactness in $L^{\infty}$.

7.1. The one-dimensional case: existence via $B V_{l o c}$ estimate. The technique of this section relies upon translation arguments for proving localized $B V$ estimates. It goes back to Bürger et al. $[13,14]$ where the idea was introduced in the context of finite volume numerical approximations.

Theorem 7.1. Assume that $\Omega=[0,+\infty)$ and $\varphi$ is locally Lipschitz continuous. Let $\beta$ be a maximal monotone graph on $\mathbb{R}$, independent of $t \in(0, T)$. Then for all $u_{0} \in L^{\infty}((0,+\infty))$ there exists an entropy solution of $\left(H_{\varphi, \beta}\left(u_{0}, f\right)\right)$ with $f=0$. 
Remark 7.2. In the case of a single boundary point $x$ and of a $t$-independent graph $\beta$, assumption (5.1) is automatically fulfilled. Indeed, in this case we can drop the subscripts $(t, x)$; the points $\pm \infty$ are accumulation points of the sets $\widetilde{D}^{ \pm}$because otherwise we have, e.g., $\varphi_{\nu}>\widetilde{\mathcal{B}}$ on $[M,+\infty)$, which contradicts the maximality of $\widetilde{\mathcal{B}}$.

Proof. According to Proposition 6.18, it is enough to prove the theorem for a dense subset of data in $L^{1}$. In order to recover existence for general $L^{\infty}$ data, we can use Proposition 6.15 applied to a bi-monotone data approximation. Uniform $L^{\infty}$ bounds are ensured by (5.1) and the assumption $f=0$, due to Remark 7.2. Substituting $\beta$ by $\widetilde{\mathcal{B}}$ as in Section 5 , we may assume that $\beta$ is bounded.

Thus we pick $u_{0} \in C^{\infty}(\Omega)$ with compact in $\mathbb{R}^{+}$support, and such that $u_{0} \equiv k_{0}=$ const on some interval $(0, \eta)$. Existence of a solution $u^{\varepsilon}$ to the parabolic regularized problem (1.3) follows by the results of $[36,37]$; we can also apply Proposition 9.6 from Appendix. Therefore assumptions (6.1),(6.3) of Theorem 6.2 hold, and it remains to guarantee (6.2) in order to apply Theorem 6.2 and conclude the proof.

To this end, we extend $u^{\varepsilon}$ continuously by $u_{0}$ for $t \leq 0$; notice that for $t<0$, the so extended function $u^{\varepsilon}$ satisfies $u_{t}^{\varepsilon}+\varphi\left(u^{\varepsilon}\right)_{x}=\varepsilon u_{x x}^{\varepsilon}+r(x)$ where

$$
r: x \mapsto \varphi\left(u_{0}\right)_{x}-\varepsilon\left(u_{0}\right)_{x x}
$$

is an $L^{\infty}(\mathbb{R}) \cap L^{1}(\mathbb{R})$ function, by the assumptions on $\varphi$ and $u_{0}$. Moreover, we can choose $k_{0} \in \widetilde{D}^{0}$, which means that $\varphi_{\nu}\left(k_{0}\right)=\widetilde{\mathcal{B}}\left(k_{0}\right)$. Therefore the so extended function $u^{\varepsilon}$ is an entire solution (i.e., a solution defined for $t \in \mathbb{R}$ ) of problem

$$
\left\{\begin{array}{l}
u_{t}^{\varepsilon}-\operatorname{div}\left(-\varphi\left(u^{\varepsilon}\right)+\varepsilon u_{x}^{\varepsilon}\right)=r(x) 1_{t<0}, \\
\left.\left(\widetilde{\mathcal{B}}\left(u^{\varepsilon}\right)+\left(-\varphi\left(u^{\varepsilon}\right)+\varepsilon u_{x}^{\varepsilon}\right) \cdot(-1)\right)\right|_{x=0}=0 .
\end{array}\right.
$$

Now, the key fact is that we can control the $L^{1}$ time translates of $u^{\varepsilon}$ by a linear modulus of continuity, because solutions of (7.2) verify the $L^{1}$ contraction principle that can be shown, e.g., as in [28] or a in [15] (we only have to take into account an original boundary condition):

$$
\int_{\mathbb{R}}\left|\tilde{u}^{\varepsilon}(t)-\tilde{u}^{\varepsilon}(t-\tau)\right| \leq \int_{\mathbb{R}}\left|\tilde{u}^{\varepsilon}(0)-\tilde{u}^{\varepsilon}(-\tau)\right|+\int_{0}^{t} \int_{\mathbb{R}}\left|r 1_{s<0}-r 1_{s-\tau<0}\right| d s=\tau\|r\|_{L^{1}} .
$$

Therefore $u^{\varepsilon} \in B V\left(0, T ; L^{1}(0,+\infty)\right)$, with a uniform in $\varepsilon$ bound. Then we can use the idea of [13, Lemma 4.2] and [14, Lemma 5.4]: for $a>0$, using the mean-value theorem for each $\varepsilon>0$ we can find a contour $(0, T) \times\left\{c^{\varepsilon}\right\}$ with $0<c^{\varepsilon}<a$ such that TotVar $u^{\varepsilon}$ along these contours is uniformly bounded by $\frac{C}{a}$. The variation of $u_{0}$ is also bounded, therefore using the classical estimate of Bardos, LeRoux and Nédélec [9] for the Dirichlet problem for viscous conservation law (with boundary datum given by the values of $u^{\varepsilon}$ on our contour), we get the bound

$$
\left.\operatorname{Tot} \operatorname{Var} u^{\varepsilon}\right|_{\{(t, x) \mid t \in(0, T), x \geq a\}} \leq \frac{C}{a},
$$

with $C$ that only depends on $u_{0}$ and on the Lipschitz constant of $\varphi$. With the Cantor diagonal argument, we deduce compactness of $\left(u^{\varepsilon}\right)_{\varepsilon}$ in $L_{l o c}^{1}((0, T) \times(0,+\infty))$. Combined with the aforementioned uniform $L^{\infty}$ bound on $u^{\varepsilon}$, this finally proves (6.2).

7.2. Entropy-process solutions. As soon as existence of an entropy solution is established ${ }^{14}$ and the uniqueness assumption (4.1) is fulfilled, we can prove convergence of, e.g., vanishing viscosity approximations without the genuine nonlinearity assumption (5.4) (though we still

\footnotetext{
${ }^{14}$ Let us stress that neither for conservation laws in the whole space, nor for the Dirichlet problem (see, e.g., Vovelle [40]) this assumption is not needed.
} 
need a uniform $L^{\infty}$ estimate). To do so, it is enough to adapt the device of measure-valued solutions; here, we use the version called entropy-process solution due to Gallouët et al. [19].

Definition 7.3. Let $\mu \in L^{\infty}\left(Q_{T} \times(0,1)\right)$. Then $\mu$ is called an entropy-process solution of problem $H_{\varphi, \beta}\left(u_{0}, 0\right)$ if $\mu$ verifies the following up-to-the-boundary entropy inequalities with remainder term (which is, in general, multi-valued):

$$
\begin{aligned}
\forall k \in \mathbb{R} \quad \forall \xi \in \mathcal{D}([0, T) \times \bar{\Omega})^{+} \quad \int_{0}^{1} \int_{0}^{T} \int_{\Omega}\left(-(\mu(\alpha)-k)^{ \pm} \xi_{t}-q^{ \pm}(\mu(\alpha), k) \cdot \nabla \xi\right) \\
-\int_{\Omega}\left(u_{0}-k\right)^{ \pm} \xi(0, \cdot) \leq \iint_{\Sigma} C_{k} \wedge\left(\beta_{(t, x)}(k)-\varphi_{\nu(x)}(k)\right)^{\mp} \xi(t, x) .
\end{aligned}
$$

Here, $C_{k}$ is a constant that depends on $\|\mu\|_{\infty}$ and on $k$.

\section{Proposition 7.4.}

(i) Let $\mu$ be an entropy-process solution of $H_{\varphi, \beta}\left(u_{0}, 0\right)$. Then it verifies the entropy-process inequalities

$$
\forall k \in \mathbb{R} \int_{0}^{1} \int_{0}^{T} \int_{\Omega}\left(-(\mu(\alpha)-k)^{ \pm} \xi_{t}-q^{ \pm}(\mu(\alpha), k) \cdot \nabla \xi\right)-\int_{\Omega}\left(u_{0}-k\right)^{ \pm} \xi(0, \cdot) \leq 0
$$

with $\xi \in \mathcal{D}([0, T) \times \Omega)^{+}$, moreover, for $\mathcal{H}^{N}$-a.e. $(t, x) \in \Sigma$, the weak normal boundary trace of the flux verifies

$$
\forall k \in D_{(t, x)}^{ \pm} \quad \gamma_{w}\left(\int_{0}^{1} q^{ \pm}(\mu(\cdot ; \alpha), k) d \alpha \cdot \nu(\cdot)\right)(t, x) \geq 0 .
$$

(ii) Let $\mu \in L^{\infty}\left(Q_{T} \times(0,1)\right)$ such that $\mu$ satisfies (7.6),(7.7). Then for $\mathcal{H}^{N}$-a.e. $(t, x) \in \Sigma$, the weak normal boundary trace of the flux verifies

$$
\forall k \in \widetilde{D}_{(t, x)}^{0} \equiv \operatorname{Dom} \tilde{\beta}_{(t, x)} \quad \gamma_{w}\left(\int_{0}^{1} q(\mu(\cdot ; \alpha), k) d \alpha \cdot \nu(\cdot)\right)(t, x) \geq 0 .
$$

(iii) Let $\mu \in L^{\infty}\left(Q_{T} \times(0,1)\right)$ such that $\mu$ satisfies (7.6),(7.8). If, in addition, (4.1) holds, then $\mu$ coincides with the entropy solution $u$ in the sense $\mu(\cdot ; \alpha)=u(\cdot)$ a.e. on $Q_{T} \times(0,1)$.

Notice that, although we do not prove directly the equivalence of Definition 7.3 and any of the formulations (7.6),(7.7) and (7.6),(7.8), such equivalence holds whenever an entropy solution exists and it is unique.

Proof.

(i) Inequalities (7.6) are immediate from the definition of an entropy-process solution. In order to deduce (7.7), one proceeds as in the proof of the claim "(iv) $\Rightarrow$ (iii)" in Proposition 3.3. The only difference is that, while using the analogue of (3.10), one replaces the (strong) trace $Q_{\nu(x)}^{ \pm}\left(\gamma V_{\varphi_{\nu(x)}} u, V_{\varphi_{\nu(x)}} k\right)$ by the (weak) trace $\gamma_{w}\left(\int_{0}^{1} q^{ \pm}(\mu(\cdot ; \alpha), k) d \alpha \cdot \nu(\cdot)\right)(t, x)$.

(ii) Assume, for instance, that $k \in D_{(t, x)}^{+}$. Let us show that (7.8) holds for this value of $k$. It is enough to prove (7.8) separately with $q^{+}$and $q^{-}$in the place of $q$; moreover, the first of the two inequalities is already contained in (7.7) since $k \in D_{(t, x)}^{+}$. Set $k_{0}:=\sup \left\{\kappa \leq k \mid \kappa \in D_{(t, x)}^{-}\right\}$; note that $k_{0}$ may take the value $-\infty$. In order to prove the statement, it is enough to get

$$
\gamma_{w}\left(\int_{0}^{1} q^{-}(\mu(\cdot ; \alpha), k) d \alpha \cdot \nu(\cdot)\right) \geq \gamma_{w}\left(\int_{0}^{1} q^{-}\left(\mu(\cdot ; \alpha), k_{0}\right) d \alpha \cdot \nu(\cdot)\right) \text { at the point }(t, x)
$$


(indeed, the latter quantity is nonnegative by $(7.7)$ because $k_{0} \in D_{(t, x)}^{-}$: recall that $D_{(t, x)}^{-}$is a closed set). Because $k \in \operatorname{Dom} \tilde{\beta}_{(t, x)}$, by Proposition 2.5(ii) we have

$$
\varphi_{\nu(x)}(\kappa) \leq \varphi_{\nu(x)}(k) \text { for all } \kappa \in\left(k_{0}, k\right) .
$$

The idea of the proof is the following: we have $q_{\nu}^{-}(\mu(\alpha), k)=-\varphi_{\nu(x)}(\mu(\alpha))+\varphi_{\nu(x)}(k) \geq 0$ whenever $\mu(\alpha) \in\left(k_{0}, k\right)$, and therefore

$$
\begin{gathered}
\int_{0}^{1} q^{-}(\mu(\alpha), k) d \alpha \cdot \nu=\int_{\left[\mu(\alpha) \leq k_{0}\right]} q_{\nu}^{-}(\mu(\alpha), k) d \alpha+\int_{\left[k_{0}<\mu(\alpha)<k\right]} q_{\nu}^{-}(\mu(\alpha), k) d \alpha+0 \\
\geq \int_{\left[\mu(\alpha) \leq k_{0}\right]} q_{\nu}^{-}\left(\mu(\alpha), k_{0}\right) d \alpha+0=\int_{0}^{1} q^{-}\left(\mu(\alpha), k_{0}\right) d \alpha \cdot \nu .
\end{gathered}
$$

Here we have used (7.10) that holds at the point $(t, x)$ but not necessarily at every point. We want to write down an inequality of the kind (7.11) in a neighbourhood $B_{\delta} \cap \Omega$ of $(t, x)$, and then take weak traces at the point $(t, x)$. In order to do so, we use an $\varepsilon$-approximate inequality of the kind (7.11) for $(s, y) \in \Omega \cap B_{\delta}$ with $B_{\delta}$ a $\delta$-sized neighbourhood of $(t, x)$, and with $\varepsilon$ vanishing as $\delta$ vanishes. This is possible due to the continuity arguments. Indeed, a generic point of $\Sigma$ is a point of approximate continuity of the normal field; thus we can write (in the place of (7.10)) that $\varphi_{\nu(y)}(\kappa) \leq \varphi_{\nu(y)}(k)+\varepsilon$ for $\kappa \in\left(k_{0}, k\right)$ and for a set of points $(s, y) \in B_{\delta} \backslash C_{\delta}$ such that meas $\left(C_{\delta}\right) / \operatorname{meas}\left(B_{\delta}\right) \rightarrow 0$ as $\delta \rightarrow 0$. Taking the weak trace, we get (7.9) with the additional term $(-\varepsilon)$ on the right-hand side. Then, letting $\delta$ go to zero, we see that (7.9) is justified and the proof is complete.

(iii) The proof is analogous to the one of Theorem 4.1, except that it is based on the doubling of variables (inside the domain) for entropy-process solutions. As in [19, 40], for entropy-process solution $\mu$ and an entropy solution $u$ corresponding to the same data, we get the Kato inequality analogous to (4.3):

$$
\forall \xi \in \mathcal{D}(\Omega) \quad \int_{0}^{1} \int_{\Omega}|\mu(\alpha)-u|(t) \xi d \alpha \leq-\int_{0}^{1} \int_{0}^{t} \int_{\Omega}(q(\mu(\alpha), u) \cdot \nabla \xi d \alpha .
$$

Assume for simplicity that $\Omega$ is bounded (other cases will exploit $\xi \in \mathcal{D}(\bar{\Omega})$ that is then sent to the limit 1 , as in $[21,11,5])$. We simply take $\xi=1-\xi_{n}$ with the construction of Lemma 3.7; as in the proof of (3.11), we deduce at the limit $n \rightarrow \infty$,

$$
\int_{0}^{1} \int_{\Omega}|\mu(\alpha)-u|(t) d \alpha \leq-\int_{0}^{t} \int_{\Omega} \gamma_{w} \int_{0}^{1} q(\mu(\alpha), u) \cdot \nu(x) d \alpha .
$$

Transforming the right-hand side of (7.13), using the existence of strong trace $\gamma V_{\varphi_{\nu(x)}} u$ we get

$$
\begin{aligned}
\int_{0}^{1} \int_{\Omega}|\mu(\alpha)-u|(t) d \alpha \leq-\int_{0}^{t} \int_{\Omega} \gamma_{w} \int_{0}^{1} Q_{\nu(x)}\left(V_{\varphi_{\nu(x)}} \mu(\alpha), V_{\varphi_{\nu(x)}} u\right) d \alpha \\
\equiv-\int_{0}^{t} \int_{\Omega} \gamma_{w} \int_{0}^{1} Q_{\nu(x)}\left(V_{\varphi_{\nu(x)}} \mu(\alpha), \gamma V_{\varphi_{\nu(x)}} u\right) d \alpha .
\end{aligned}
$$

Yet, according to the characterization Proposition 3.3(i) of $u, \gamma V_{\varphi_{\nu(x)}} u \in \operatorname{Dom} \tilde{\beta}_{(t, x)}$ in a generic point of $\Sigma$; thus using (7.8) (notice that one can replace $q(\mu(\alpha), k) \cdot \nu(x)$ in $(7.8)$ by the expression $\left.Q_{\nu(x)}\left(V_{\varphi_{\nu(x)}} \mu(\alpha), V_{\varphi_{\nu(x)}} k\right)\right)$ we readily find that the right-hand side of (7.14) is non-positive. It follows that $\mu(\alpha)-u$ is zero a.e., which ends the proof.

Corollary 7.5. In the assumptions of Theorem 6.2, drop the genuine nonlinearity assumption (5.4) but suppose that there exists an entropy solution of problem $\left(H_{\varphi, \beta}\left(u_{0}, f\right)\right)$. Then the conclusion of the theorem still holds. 
For the proof, it is enough to use the device of nonlinear weak-* convergence, following [19, 40], to derive the entropy-process formulation (7.5) along the lines of the proof of Theorem 6.2. One concludes using Proposition 7.4(i)-(iii).

\section{CONCLUSION}

We investigated the issue of definition, justification and uniqueness of entropy solutions to scalar conservation laws with nonlinear dissipative boundary conditions. Although existence of entropy solutions and convergence of approximations are addressed in much generality, technical restrictions we had to impose leave place for a future work, e.g., exploiting the notions of renormalized entropy solutions $([10,34,2])$ and of weak boundary traces and boundary entropy-flux pairs ([27]), as it was done for the Dirichlet problem.

\section{APPENDIX: EXISTENCE FOR THE VISCOSITY REGULARIZED PROBLEM}

In this paper, we establish existence of entropy solutions of $\left(H_{\varphi, \beta}\left(u_{0}, f\right)\right)$ via construction of approximate solutions (in most cases, we need a multi-step approximation). Therefore we need some basic existence result to produce approximate solutions; this is the purpose of the present Appendix. Existence results of this kind were already established by the second author and Wittbold in [37] (see also [36]), for the case of $t$-independent graph $\beta$ such that $0 \in \beta(0)$. Other results can be found in [4].

Here we follow a different strategy (in the place of the convex analysis and nonlinear semigroup methods of $[37,36]$, we use the Galerkin scheme and time compactness arguments), in the context that suits more our needs. Consider the following parabolic problem (for simplicity, we set $f \equiv 0$ ):

$$
\left\{\begin{array}{l}
u_{t}-\operatorname{div}(-\varphi(u)+\varepsilon \nabla u)=0,\left.\quad u\right|_{t=0}=u_{0}, \\
b(t, x ; u)+(-\varphi(u)+\varepsilon \nabla u) \cdot \nu(x)=0
\end{array}\right.
$$

where $b$ is a Caratheodory function ( $\operatorname{single-valued} b(t, x ; \cdot)$ replaces the maximal monotone graph $\left.\beta_{(t, x)}\right)$, more precisely

for all $z \in \mathbb{R} \quad b(\cdot, \cdot ; u)$ is measurable,

and for a.e. $(t, x) \in \Sigma \quad b(t, x ; \cdot)$ is a continuous strictly increasing function.

Moreover, we assume that $b$ is bounded:

$$
\sup _{(t, x) \in \Sigma, z \in \mathbb{R}}|b(t, x ; z)|<+\infty .
$$

The parameter $\varepsilon$ in (9.15) could be removed by a scaling argument, but we keep it in order to state an $\varepsilon$-independent $L^{\infty}$ estimate on $u^{\varepsilon}$ that is needed in order to generate a limit of the sequence $\left(u^{\varepsilon}\right)_{\varepsilon}$, as $\varepsilon \rightarrow 0$.

\section{Proposition 9.6.}

(i) Under the above assumptions, suppose in addition that $\varphi$ is bounded on $\mathbb{R}$. Then there exists a solution $u^{\varepsilon}$ to problem (9.15): namely, $u^{\varepsilon} \in L^{2}\left(0, T ; H_{l o c}^{1}(\Omega)\right)$ and for all $\xi \in \mathcal{D}([0, T) \times \Omega)$

$$
\int_{0}^{T} \int_{\Omega}-u \xi_{t}-\int_{\Omega} u_{0} \xi(0, \cdot)+\int_{0}^{T} \int_{\Omega}(-\varphi(u)+\varepsilon \nabla u) \cdot \nabla \xi+\int_{0}^{T} \int_{\partial \Omega} b(\cdot ; u) \xi=0 .
$$

Moreover, $\sqrt{\varepsilon} \nabla u^{\varepsilon}$ is bounded in $L^{2}\left(0, T ; L_{\text {loc }}^{2}(\Omega)\right)$ uniformly in $\varepsilon \in(0,1)$.

(ii) Under the assumption that, upon a modification of b on a subset of $\Sigma$ of zero $\mathcal{H}^{N}$ measure,

$$
\begin{aligned}
& \text { there exist }\left(A_{m}^{-}\right)_{m \in \mathbb{N}},\left(A_{m}^{+}\right)_{m \in \mathbb{N}} \subset \mathbb{R}^{ \pm} \text {such that } A_{m}^{ \pm} \rightarrow \pm \infty \\
& \text { as } m \rightarrow \infty \text { and for all }(t, x) \in \Sigma \text {, for all } m \in \mathbb{N} \quad \pm b\left(t, x ; A_{m}^{ \pm}\right) \geq \pm \varphi_{\nu(x)}\left(A_{m}^{ \pm}\right)
\end{aligned}
$$


we have, uniformly in $\varepsilon>0$, the estimate $\left\|u^{\varepsilon}\right\|_{\infty} \leq M$ with $M$ that depends on $\left\|u_{0}\right\|_{\infty}$ and on $\left(A_{m}^{ \pm}\right)_{m}$ in assumption $(9.17)$.

Remark 9.7. For non-zero $f$, existence is shown in the same way. In property (ii), hypothesis

$$
\mathcal{A}^{ \pm}:=\left\{k \in \mathbb{R}^{ \pm} \mid \pm b(t, x ; k) \geq \pm \varphi_{\nu(x)}(k) \text { for all }(t, x) \in \Sigma\right\} \text { are of infinite measure }
$$

can be assumed in the place of (9.17), and the bound $M$ would depend on $\left\|u_{0}\right\|_{\infty}, \int_{0}^{T}\|f(t, \cdot)\|_{\infty} d t$ and on the sets $\mathcal{A}^{ \pm}$in assumption (9.18).

In the place of a constant in $t$ and $x$ sub- and super-solutions, in this case we construct supersolutions of the kind $M^{ \pm}(t)$ taking values in $\mathcal{A}^{ \pm}$and such that $\pm M^{ \pm}(\cdot)$ are non-decreasing, with $\pm M^{ \pm}(0) \geq\left\|u_{0}\right\|_{\infty}$ and with the absolutely continuous part of the derivative $\pm\left(M^{ \pm}\right)^{\prime}(t)$ that is greater than or equal to $\|f(t, \cdot)\|_{\infty}$ on $(0, T)$.

Proof. For the proof, it is enough to use the Galerkin method, which we expose briefly in order to focus on the difficulties induced by the non-linearities $\varphi$ and $b$.

In the case $\Omega$ is bounded, picking an orthonormal basis $\left(v_{i}\right)_{i \in \mathbb{N}}$ in $H^{1}(\Omega)$, we construct $u_{n} \in$ $C^{1}\left([0, T] ; \operatorname{span}\left\{v_{1}, \ldots, v_{n}\right\}\right)$ as a solution to the ODE system obtained from (9.16) by substituting $u$ by the unknown function $u_{n}(t, x)=\sum_{i=1}^{n} c_{i}(t) v_{i}(x)$, substituting $u_{0}$ by its projection $u_{0, n}$ on $\operatorname{span}\left\{v_{1}, \ldots, v_{n}\right\}$, and testing it with $\xi(t, x)=v_{i}(x) \mu(t), i=1, \ldots, n, \mu \in \mathcal{D}([0, T))$. Local existence of a solution follows from the Cauchy-Peano theorem. Taking $u_{n}$ itself for the test function, with $\mu(t)$ approximating $\mathbb{1}_{[0, s)}(t)$ we find

$$
\frac{1}{2} \int_{\Omega} u_{n}^{2}(s, \cdot)+\int_{0}^{s} \int_{\Omega}\left(\varepsilon\left|\nabla u_{n}\right|^{2}-\varphi\left(u_{n}\right) \cdot \nabla u_{n}\right)+\int_{0}^{s} \int_{\partial \Omega} b\left(\cdot ; u_{n}\right) u_{n}=\frac{1}{2} \int_{\Omega} u_{0, n}^{2} \leq \frac{1}{2} \int_{\Omega} u_{0}^{2} .
$$

Thanks to trace inequalities and the boundedness assumptions on $\varphi$ and $b$ together with the $L^{\infty}$ bound on $u_{0}$ we get an $L^{2}\left(Q_{T}\right)$ estimate on $\nabla u_{n}$. Such estimate precludes the blow-up and guarantees the global in time existence of $u_{n}$. For the case of unbounded domain, the mere $L^{\infty}$ bounds on $u_{0}, \varphi, b$ are not sufficient: thus we have to localize the estimate taking e.g. the weight $\eta(x)=\exp \left(-c\left|x-x_{0}\right|\right)$ for some $x_{0} \notin \bar{\Omega}$. In this case, we work in the weighted $H^{1}$ space and use weighted trace inequalities; as an outcome, we get an $L^{2}\left(0, T ; L_{l o c}^{2}(\Omega)\right)$ bound on $u_{n}$.

Thus we have, in addition, the uniform in $n$ estimate of $\nabla u_{n}$ in $L^{2}\left(0, T ; H_{l o c}^{1}(\Omega)\right)$. We extract a subsequence weakly convergent to a limit $u^{\varepsilon}$, and pass to the limit in the formulation. To this end, the a.e. convergence of $u_{n}$ to $u^{\varepsilon}$ is needed in order to pass to the limit in the nonlinearity $\varphi\left(u_{n}\right)$. It is obtained by translation techniques in time, following [1]. Indeed, assume for simplicity that $\Omega$ is bounded (otherwise we use exponentially decreasing in $x$ weights, as above). We "integrate" the weak formulation (9.16) from $t$ to $t+\delta t$, then test it with $\xi=u_{n}(t+\delta t)-u_{n}(t)$ (this corresponds to taking well-chosen test functions in the formulation (9.16) written for $u_{n}$ and with test functions $\left.\xi \in \mathcal{D}\left([0, T) ; \operatorname{span}\left\{v_{1}, \ldots, v_{n}\right\}\right)\right)$. Using the Fubini theorem, the aforementioned $L^{2}\left(0, T ; H^{1}(\Omega)\right)$ bound on $u_{n}$, the trace inequality and the $L^{\infty}$ bound on $\varphi\left(u_{n}\right)$ and $b\left(\cdot ; u_{n}\right)$, we deduce that

$$
\int_{0}^{T-\delta t}\left|u_{n}(t+\delta t)-u_{n}(t)\right|^{2} \leq \text { const } \delta t .
$$

The estimate of the space translates being trivial due to the $L^{2}\left(Q_{T}\right)$ bound on $\nabla u_{n}$, by the Fréchet-Kolmogorov compactness criterion we conclude to the $L^{1}\left(Q_{T}\right)$ convergence of $u_{n}$ (if $\Omega$ is unbounded, we use weights $\eta$ and get $L^{1}\left((0, T) ; L_{l o c}^{1}(\Omega)\right)$ convergence). The limit being unique in $\mathcal{D}^{\prime}$, it is identified with $u_{\varepsilon}$; extracting a further subsequence, we may assume the a.e. convergence in $Q_{T}$. Finally, $\left(b\left(\cdot ; u_{n}\right)\right)_{n}$ being bounded, it converges weakly-* in $L^{\infty}(\Sigma)$ to some limit that we denote by $b^{\varepsilon}$. Now for all $i$, we can take $v_{i}$ as a test function and pass to the limit in the 
Galerkin formulation; we find that

$$
\int_{0}^{T} \int_{\Omega}-u^{\varepsilon} \xi_{t}-\int_{\Omega} u_{0} \xi(0, \cdot)+\int_{0}^{T} \int_{\Omega}\left(-\varphi\left(u^{\varepsilon}\right)+\varepsilon \nabla u^{\varepsilon}\right) \cdot \nabla \xi+\int_{0}^{T} \int_{\partial \Omega} b^{\varepsilon} \xi=0
$$

for all $\xi \in \mathcal{D}^{\prime}([0, T) \times \Omega)$ (this is obtained by density). It remains to identify $b^{\varepsilon}$ with $b\left(\cdot ; u^{\varepsilon}\right)$, which is done using the monotonicity of $b(t, x ; \cdot)$ and the classical Minty argument.

To do so, for the sake of simplicity assume that $\Omega$ is bounded (for the general case, one has to replace the test functions $u^{\varepsilon}, u_{n}$ below by truncated test functions $u^{\varepsilon} \eta, u_{n} \eta$ with an exponentially decaying weight $\eta$ ). Comparing the Galerkin formulation for $u_{n}$ (with test function $u_{n}$ ) and the weak formulation (9.20) for $u^{\varepsilon}$ (with test function $u^{\varepsilon}$, taken by density), using the Fatou lemma to ensure that $\left\|u^{\varepsilon}(T, \cdot)\right\|_{L^{2}(\Omega)}^{2} \leq \liminf _{n \rightarrow \infty}\left\|u_{n}(T, \cdot)\right\|_{L^{2}(\Omega)}^{2}$, we can eventually write

$$
\int_{0}^{T} \int_{\partial \Omega} b^{\varepsilon} u^{\varepsilon}+\varepsilon \int_{0}^{T} \int_{\Omega}\left|\nabla u^{\varepsilon}\right|^{2} \geq \limsup _{n \rightarrow \infty}\left(\int_{0}^{T} \int_{\partial \Omega} b\left(\cdot ; u_{n}\right) u_{n}+\varepsilon \int_{0}^{T} \int_{\Omega}\left|\nabla u_{n}\right|^{2}\right) .
$$

Due to the weak lower semicontinuity of the $L^{2}$ norm, we infer

$$
\int_{0}^{T} \int_{\partial \Omega} b^{\varepsilon} u^{\varepsilon} \geq \limsup _{n \rightarrow \infty} \int_{0}^{T} \int_{\partial \Omega} b\left(\cdot ; u_{n}\right) u_{n}
$$

Here we are in the following setting: $\left.u_{n}\right|_{\Sigma}$ converges to $\left.u^{\varepsilon}\right|_{\Sigma}$ in $L^{2}(\Sigma)$ weakly (this is due to the trace inequalities); $b\left(\cdot ; u_{n}\right)$ converges to $b^{\varepsilon}$ in $L^{\infty}(\Sigma)$ weakly-* (and thus, in $L^{2}(\Sigma)$ weakly, because we have assumed that $\partial \Omega$ is bounded); moreover, $z \mapsto b(\cdot ; z)$ is monotone and inequality (9.22) holds. In this setting, the Minty argument applies (see, e.g., [1, 28, 37]) which allows to conclude that $b^{\varepsilon}=b\left(\cdot ; u^{\varepsilon}\right)$ a.e. on $\Sigma$. Thus (9.20) becomes (9.16), and the proof of existence is complete.

(ii) Now using (9.17), take $A_{m}^{ \pm}$satisfying $A_{m}^{-} \leq-\left\|u_{0}\right\|_{\infty}$ and $\left\|u_{0}\right\|_{\infty} \leq A_{m}^{+}$; due to (9.17), the constants $A_{m}^{-}$and $A_{m}^{+}$are sub- and super-solutions to problem (9.15), respectively. The result stems from the comparison principle for weak solutions, sub- and super-solutions of (9.15) using, e.g., the technique of [15]. It consists in taking $H_{\alpha}\left(u^{\varepsilon}-A_{m}^{+}\right) \xi$ (with $H_{\alpha}$ the Lipschitz regularization of sign ${ }^{+}$function as used in the proof of Theorem 6.2$)$ with $\xi \in \mathcal{D}([0, T) \times \bar{\Omega})^{+}$; the factor $\xi$ can be dropped if $\Omega$ is bounded. As in the proof of Theorem 6.2, we deduce the Kato inequality

$$
-\int_{0}^{T} \int_{\Omega}\left(u^{\varepsilon}-A_{m}^{+}\right)^{+} \xi_{t}+\int_{0}^{T} \int_{\Omega} \operatorname{sign}^{+}\left(u^{\varepsilon}-A_{m}^{+}\right)\left(-\varphi\left(u^{\varepsilon}\right)+\varphi\left(A_{m}^{+}\right)+\varepsilon \nabla u^{\varepsilon}\right) \cdot \nabla \xi \leq 0 .
$$

We let $\xi$ converge to $e^{-t}$ and prove that $\left(u^{\varepsilon}-A_{m}^{+}\right)^{+} \leq 0$ a.e. (if $\Omega$ is unbounded, we use (4.1) as in $[25,5])$. A uniform upper bound for $u^{\varepsilon}$ is proved; the lower bound by $A_{m}^{-}$is analogous.

Notice that the technique we've used exploits assumption (4.1); yet it is possible to bypass this assumption. Indeed, by approximation one can always construct solutions satisfying the above $L^{\infty}$ bound. To this end, one can, e.g., substitute the original problem by the problem set up in $(0, T) \times\left(\Omega \cap B_{R}\right)$ where $B_{R}$ is the ball of radius $R$ centered at the origin; the part $\Sigma_{R}^{\prime}:=(0, T) \times \partial B_{R} \cap \Omega$ of the boundary can be supplemented with the homogeneous Dirichlet boundary condition. Then existence of solutions $u_{R}$ in the space $L^{2}\left(0, T ; H_{0, R}^{1}(\Omega)\right)$ of functions that are zero in $(0, T) \times\left(\Omega \backslash B_{R}\right)$ is proved by the same Galerkin method. Notice that the constants $A_{m}^{ \pm}$are still sub- and super-solutions of this modified problem; $B_{R}$ being bounded, assumption (4.1) is automatically satisfied and the $L^{\infty}$ bound on $u_{R}$ is valid. Finally, convergence of $u_{R}$ to a limit $u^{\varepsilon}$ is established with the same tools as in the proof of (i).

Remark 9.8. While estimating $\nabla u^{\varepsilon}$ in $L_{l o c}^{2}$, for the sake of simplicity we have assumed that $b$ is bounded and thus we have not exploited the monotonicity of $b$ in these estimates. Actually, it is enough to assume, e.g., that $b(t, x ; 0)$ is bounded; in addition, estimate $(9.19)$ brings an 
$L_{l o c}^{1}([0, T] \times \partial \Omega)$ estimate of the product $\left(b\left(\cdot ; u_{n}\right)-b(t, x ; 0)\right) u_{n} \geq 0$, which is inherited at the limit $u_{n} \rightarrow u^{\varepsilon}$. Similarly, instead of the uniform bound on $\varphi$ we could assume inequalities of the kind $\left|\int_{0}^{z} \varphi(s) d s\right| \leq C+\operatorname{sign} z\left(b\left(t, x^{\prime} z\right)-b(t, x ; 0)\right)$.

\section{REFERENCES}

[1] H. W. Alt and S. Luckhaus, Quasilinear elliptic-parabolic differential equations, Math. Z. 183 (1983), no.3, pp.311-341. MR0706391

[2] K. Ammar, J. Carrillo, and P. Wittbold, Scalar conservation laws with general boundary condition and continuous flux function, J. Diff. Equ. 228 (2006), no.1, pp.111-139. MR2254186

[3] F. Andreu, N. Igbida, J. Mazón, and J. Toledo, $L^{1}$ Existence and Uniqueness Results for Quasi-linear Elliptic Equations with Nonlinear Boundary Conditions, Annales de l'IHP (C) : Non Linear Anal. 24 (2007), no.1, pp.61-89. MR2286559

[4] F. Andreu, N. Igbida, J. Mazón, and J. Toledo, Renormalized solutions for degenerate elliptic-parabolic problems with nonlinear dynamical boundary conditions and L1-data, J. Differ. Equ. 244 (2008), no.11, pp.2764-2803. MR2418175

[5] B. Andreianov and M. Maliki, A note on uniqueness of entropy solutions to degenerate parabolic equations in $\mathbb{R}^{N}$, NoDEA Nonlinear Diff. Equ. Appl. 17 (2010),no.1, pp.109-118. MR2596497

[6] B. Andreianov and K. Sbihi, Strong boundary traces and well-posedness for scalar conservation laws with dissipative boundary conditions. Hyperbolic problems: theory, numerics, applications (Proc. of the HYP2006 Conference, Lyon), Springer, Berlin, pp.937-945, 2008. MR2549233

[7] B. Andreianov and K. Sbihi, Scalar conservation laws with nonlinear boundary conditions, C. R. Acad. Sci. Paris, Ser. I 345 (2007), pp.431-434. MR2367360

[8] J. M. Ball and F. Murat, Remarks on Chacon's biting lemma, Proc. of AMS 107 (1989), no.3, pp.655-663. MR0984807

[9] C. Bardos, A.Y. Le Roux, and J.-C. Nédélec, First order quasilinear equations with boundary conditions, Comm. Partial Diff. Equ. 4 (1979), no.4, pp.1017-1034. MR0542510

[10] Ph. Bénilan, J. Carrillo, and P. Wittbold, Renormalized entropy solutions of scalar conservation laws, Ann. Scuola Norm. Sup. Pisa Cl. Sci. (4) 29 (2000), no.2, pp.313-327. MR1784177

[11] Ph. Bénilan and S.N. Kruzhkov. Conservation laws with continuous flux functions, NoDEA Nonlinear Diff. Equ. Appl. 3 (1996), no.4, pp.395-419. MR1418588

[12] R. Bürger, H. Frid, and K.H. Karlsen. On the well-posedness of entropy solutions to conservation laws with a zero-flux boundary condition, J. Math. Anal. Appl. 326 (2007), pp.108-120. MR2277770

[13] R. Bürger, A. García, K.H. Karlsen, and J.D. Towers, A family of numerical schemes for kinematic flows with discontinuous flux, J. Engrg. Math. 60 (2008), no.3-4, pp.387-425. MR2396491

[14] R. Bürger, K. H. Karlsen, and J. D. Towers, An Engquist-Osher-type scheme for conservation laws with discontinuous flux adapted to flux connections, SIAM J. Numer. Analysis, 47 (2009), no.3, pp.1684-1712. MR2505870

[15] J. Carrillo, Entropy solutions for nonlinear degenerate problems. Arch. Ration. Mech. Anal. 147 (1999), no.4, pp.269-361. MR1709116

[16] G.-Q. Chen and H. Frid. Divergence-Measure fields and hyperbolic conservation laws, Arch. Ration. Mech. Anal. 147 (1999), pp.89-118. MR1702637

[17] F. Dubois and Ph. LeFloch, Boundary conditions for nonlinear hyperbolic systems of conservation laws, J. Differ. Equ. 71 (1988), no.1, pp.93-122. MR0922200

[18] L.C. Evans and R.F. Gariepy, Measure theory and fine properties of functions. Studies in Advanced Mathematics. CRC Press, Boca Raton, 1992. MR1158660

[19] R. Eymard, Th. Gallouët, and R. Herbin, Existence and uniqueness of entropy solution to a nonlinear hyperbolic equation, Chin. Ann of Math. 16B (1995), no.1, pp.1-14. MR1338923

[20] S.N. Kruzhkov, First order quasilinear equations with several independent variables, Mat. Sb. 81(123) (1970), pp.228-255. MR0267257

[21] S.N. Kruzhkov and E.Yu. Panov, Osgood's type conditions for uniqueness of entropy solutions to Cauchy problem for quasilinear conservation laws of the first order, Ann. Univ. Ferrara, 15 (1994), pp.31-53. MR1399621

[22] Y.S. Kwon and A. Vasseur, Strong traces for solutions to scalar conservation laws with general flux, Arch. Ration. Mech. Anal. 185 (2007), no.3, pp.495-513. MR2322819 
[23] P.L. Lions, B. Perthame, and E. Tadmor, A kinetic formulation of multidimensional scalar conservation laws and related equations, J. Amer. Math. Soc. 7 (1994), no.1, pp.169-191. MR1201239

[24] J. Málek, J. Necăs, M. Rokyta, and M. Ružička. Weak and Measure-valued Solutions to Evolutionnary PDEs, Applied Mathematics and Mathematical Computation 13, Chapman \& Hall, 1996. MR1409366

[25] M. Maliki and H. Touré, Uniqueness of entropy solutions for nonlinear degenerate parabolic problem, J. Evol. Equ. 3 (2003), no.4, pp.603-622. MR2058053

[26] F. Otto, Ein Randwertproblem für skalare Erhaltnungssätze. (German) PhD thesis, Universität Bonn, 1993.

[27] F. Otto, Initial-boundary value problem for a scalar conservation law, C. R. Acad. Sci. Paris Sér. I Math. 322 (1996), pp.729-734. MR1387428

[28] F. Otto, $L^{1}$-contraction and uniqueness for quasilinear elliptic-parabolic equations, J. Differ. Equ. 131 (1996), no.1, pp.20-38. MR1415045

[29] E.Yu. Panov, On sequences of measure-valued solutions of a first-order quasilinear equation, Mat. Sb. 185 (1994), no.2, 87-106; translation in Russian Acad. Sci. Sb. Math. 81 (1995), no.1, pp.211-227. MR1264775

[30] E.Yu. Panov, Existence of strong traces for generalized solutions of multidimensional scalar conservation laws, J. Hyperbolic Differ. Equ. 2 (2005), no.4, pp.885-908. MR2195985

[31] E.Yu. Panov, Existence of strong traces for quasi-solutions of multidimensional scalar conservation laws, J. Hyperbolic Differ. Equ. 4 (1997), no.4, pp.729-770. MR2374223

[32] E.Yu. Panov, Existence and strong pre-compactness properties for entropy solutions of a first-order quasilinear equation with discontinuous flux, Arch. Ration. Mech. Anal. 195 (2010), no.2, pp.643-673. MR2592291

[33] B. Perthame, Kinetic formulation of conservation laws. Oxford Lecture Series in Math. and Appl. 21. Oxford Univ. Press, 2002. MR2064166

[34] A. Porretta and J. Vovelle, $L^{1}$ solutions to first order hyperbolic equations in bounded domains, Comm. Partial Differ. Equ. 28 (2003), no.1-2, pp.381-408. MR1974461

[35] E. Rouvre and G. Gagneux, Formulation forte entropique de lois scalaires hyperboliques-paraboliques dégénérées (French) [Strong entropy formulation of degenerate hyperbolic-parabolic scalar laws], Ann. Fac. Sci. Toulouse Math. (6) 10 (2001), no.1, pp.163-183. MR1928992

[36] K. Sbihi, Etude de quelques E.D.P. non linéaires dans $L^{1}$ avec des conditions générales sur le bord (French, English) [Study of some nonlinear PDEs in $L^{1}$ with general boundary conditions], PhD Thesis, University of Strasbourg, France (2006). Available at http://tel.archives-ouvertes.fr/tel-00110417

[37] K. Sbihi and P. Wittbold, Entropy solution of a quasilinear elliptic problem with nonlinear boundary condition, Commun. Appl. Anal. 11 (2007), no.2, pp.299-325. MR2371480

[38] G. Vallet, Dirichlet problem for a degenerated hyperbolic-parabolic equation, Adv. Math. Sci. Appl. 15 (2005), no.2, pp.423-450. MR2198570

[39] A. Vasseur, Strong traces for weak solutions to multidimensional conservation laws, Arch. Ration. Mech. Anal. 160 (2001), no.3, pp.181-193. MR1869441

[40] J. Vovelle. Convergence of finite volume monotone schemes for scalar conservation laws on bounded domains, Numer. Math. 90 (2002), no.3, pp.563-596. MR1884231

Laboratoire de Mathématiques CNRS UMR 6623, Université de Franche-Comté, 16 route de Gray, 25030 Besançon Cedex, France

Current address: Laboratoire de Mathématiques CNRS UMR 6623, Université de Franche-Comté, 16 route de Gray, 25030 Besançon Cedex, France

E-mail address: boris.andreianov@univ-fcomte.fr

Laboratoire de Mathématiques CNRS UMR 6623, Université de Franche-Comté, 16 route de Gray, 25030 Besançon Cedex, France

Current address: Laboratoire de Mathématiques CNRS UMR 6623, Université de Franche-Comté, 16 route de Gray, 25030 Besançon Cedex, France

E-mail address: sbihi_k@yahoo.fr 